\title{
Crack tip fields in soft elastic solids subjected to large quasi-static deformation $-\mathbf{A}$ review
}

Rong Long ${ }^{1}$ and Chung-Yuen Hui ${ }^{2}$

${ }^{1}$ Department of Mechanical Engineering, University of Colorado Boulder, Boulder, CO 80309, USA.

${ }^{2}$ Field of Theoretical and Applied Mechanics, Department of Mechanical and Aerospace Engineering, Cornell University, Ithaca, NY 14853, USA.

\begin{abstract}
:
We review analytical solutions for the asymptotic deformation and stress fields near the tip of a crack in soft elastic solids. These solutions are based on finite strain elastostatics and hyperelastic material models, and exhibit significantly different characteristics than the classical crack tip field solutions in linear elastic fracture mechanics. Specifically, we summarize some available finite strain crack tip solutions for two dimensional cracks, namely that plane strain, plane stress, and anti-plane shear cracks in a certain class of homogeneous materials. Interface cracks between soft elastic solids and a rigid substrate are also discussed. We focus on incompressible material models with various degrees of strain stiffening effect such as generalized neo-Hookean model, exponentially hardening model and Gent model. We also explored the physical implications of the crack tip fields, and highlighted pitfalls in the applications of these solutions, particularly the J-integral and the distribution of true stress in the deformed configuration which have not been discussed in the literature.
\end{abstract}

Keywords: finite strain, crack tip fields, asymptotic analysis, hyperelasticity, strain hardening. 


\section{List of main symbols and abbreviations}

$\eta_{1}(\theta, n): \quad$ Dimensionless function in (44b)

$\eta_{1}(\theta, n): \quad$ Dimensionless function in (44c)

$\kappa: \quad \kappa=1-1 / n$ in $(36 b)$.

$\mu$ : $\quad$ Small strain shear modulus

$(\rho, \phi): \quad$ Polar coordinates at deformed crack tip (see Fig.1b)

$\sigma_{i j}\left(\sigma_{\alpha \beta}\right): \quad$ Nominal stress or the first Piola-Kirchhoff stress

$\tau_{i j}\left(\tau_{\alpha \beta}\right): \quad$ True stress or Cauchy stress

$\omega(\theta, n) \quad$ Dimensionless function in (36c)

$\xi_{0}: \quad$ Intermediate variable defined in (37c)

$A, B: \quad$ Coefficients of strain energy density in plane strain (see (31))

$A_{2}: \quad$ Coefficient in plane stress crack tip solution for exponential solids (see (69a))

$a: \quad$ Coefficient in crack tip solution (see (35b), (58b),(76))

$b_{0} \quad$ Coefficient in plane strain crack tip solution (see (35a))

b: $\quad$ Material parameter in the generalized neo-Hookean model (see (13))

$c: \quad$ Coefficient in plane stress crack tip solution (see (58a))

$d: \quad$ Exponent of the crack tip solution of $y_{1}$ in plane stress (see (58a))

$F_{\alpha \beta}: \quad$ 2-D deformation gradient

EM: $\quad$ Exponential Hardening Model introduced in (14)

FEM: $\quad$ Finite Element Method

GM: $\quad$ Gent Model introduced in (15)

GNM: $\quad$ Generalized neo-Hookean Model introduced in (13)

$g(\theta, n)$ : Dimensionless function describing the angular variation of $y_{1}$ in plane stress (see $(58 \mathrm{a}))$

$H(\theta, n): \quad$ Dimensionless function introduced in (37a)

$\hat{I}_{n}(\theta): \quad$ Dimensionless function introduced in $(44 \mathrm{a})$

$I_{1}=I: \quad$ First invariant of the right Cauchy-Green tensor $C_{i j}($ see (6)) 


$$
\begin{array}{ll}
I_{2}: & \text { Second invariant of the right Cauchy-Green tensor (see (6)) } \\
J: & \text { J-integral defined in (88a,b) } \\
J_{n}: & \text { Material parameter in the exponential model or Gent solid (see (14) and (15)) } \\
K_{I}, K_{I I}, K_{I I I}: & \text { Mode I, II and III stress intensity factors in linear elastic fracture mechanics } \\
\text { LEFM } & \text { Linear Elastic Fracture Mechanics } \\
m: & m=1-1 /(2 n) \text { in (37b) } \\
n: & \text { Material parameter describing the degree of strain hardening (see (13) and (34)) } \\
n^{*}: & \text { Critical strain hardening exponent at which the plane stress crack tip field changes } \\
p: & \text { behavior (see (58a) and } \left.n^{*} \approx 1.46\right) . \\
P(\theta, n): & \text { Pressure required to enforce incompressibility } \\
(r, \theta): & \text { Angular variation of the pressure fields for 1/2 <n<3/2 in (44a). } \\
U(\theta, n): & \text { Dimensionless function introduced in (36a) } \\
u_{i}: & \text { Components of the displacement of a material point } \\
W: & \text { Strain energy density } \\
X_{\alpha}: & \text { Material coordinates (see Fig.1) } \\
x_{\alpha}: & \text { Deformed coordinates with origin at undeformed crack tip (see Fig.1) } \\
y_{\alpha}: & \text { Deformed coordinates with origin at deformed crack tip (see Fig.1) } \\
y_{\alpha}^{*}: & \text { Canonical form of mixed-mode crack tip solutions (see (55)) }
\end{array}
$$




\section{Introduction}

Studies on the fracture of soft materials can be dated back to the 1950s where the first attempt to quantitatively characterize and understand the rupture behaviors of rubber was made in a number of seminal works [1, 2, 3, 4, 5]. Rivlin and Thomas [1] conducted fracture experiments on natural rubber and found that the critical fracture load for various testing configurations and sample geometries was dictated by a single characteristic tearing energy, or fracture energy. Based on this finding, they proposed an energy based fracture criterion where the onset of growth of a pre-existing crack occurs when the applied energy release rate is equal to a characteristic energy/area which in modern terms is called fracture energy - a characteristic of the material. Numerous experiments were carried out to measure the fracture energy of rubbers and the results indicated strong rate and temperature dependency for the fracture energy [6], which are commonly attributed to energy dissipation due to viscoelasticity. Recently, interest in soft material fracture has been renewed by the development of hydrogels $[7,8,9,10,11]$ or polymers [12] with high extensibility and fracture toughness. Examples include the doublenetwork gel [7], the hybrid polyacrylamide-alginate gel [8] and the ionically crosslinked triblock copolymer gel [10]. These soft and yet tough materials, when combined with additional functionalities, have great potentials in a wide range of applications such as bio-adhesives [13], biomedical implants [14], tissue engineering [15], and soft actuators [16].

The most common way to test the fracture behaviors of soft materials is to first introduce a sharp crack in a specimen and then mechanically load the specimen until the crack propagates forward. Compared to other fracture tests which relies on the presence of initial defects to induce local failure mechanisms such as cavitation [17-19], the crack geometry provides a well-defined initial configuration where the stress distribution near the crack tip is similar, independent of geometry. As long as the deviation from the continuum description occurs over a region small in comparison with typical specimen dimensions, the amplitude of the near tip stress fields controls the local failure processes such as breaking of polymer chains, and growth and coalescence of cavities. However, unlike stiff materials such as metal and ceramics, cracks in soft and tough materials can become highly deformed and blunted before propagation [20]. This can be further complicated by time-dependent effects such as viscoelasticity [6] and poroelasticity [21, 22]. Therefore, the classical solutions for crack tip fields in linear elastic fracture mechanics (LEFM), or the $K$-field [23], which were based on the assumption of infinitesimal deformation, are not sufficient to describe the local crack tip stress field in soft materials. For example, experimental 
observations and theoretical analyses suggested that the LEFM crack tip fields broke down for dynamic $[24,25,26,27,28]$ or quasi-static cracks $[25,28,29]$ in soft polymeric gels.

A first step towards understanding crack tip fields in soft materials is to take large deformation effects into consideration by adopting the framework of finite strain elastostatics. The earliest effort along this direction was due to Wong and Shield [30], where closed form approximate solutions for a plane stress crack in a sheet of incompressible neo-Hookean material were presented. After that, Knowles and Sternberg [31, 32] performed the first systematic asymptotic analysis of near-tip fields for a plane strain crack under symmetric opening deformation (Mode-I). They considered a class of power-law stiffening compressible hyperelastic solids, and show that the severity of the singular stress and deformation fields depends on material stiffening. The amplitudes of these singular stress and deformation fields are governed by far-field loading conditions and are analogous to the stress intensity factors in LEFM [33, 23]. These pioneering works inspired further studies to understand the local stress and deformation fields near cracks in homogeneous hyper-elastic solids, which can be roughly divided into five categories: plane strain cracks in compressible materials [34, 35, 36, 37, 38], plane strain cracks in incompressible materials [39, 40,41], plane stress cracks in compressible materials [42, 43, 44], plane stress cracks in incompressible materials [45, 46], and anti-plane shear cracks (Mode III) [47, 48]. In addition, a number of analyses were devoted to interface cracks between two dissimilar hyperelastic materials, either in plane stress $[49,50,51]$ or in plane strain $[52,53,54,55]$ condition. A narrative of these works can be found in Gao et al. [56]. Finite element analysis (FEA) has also been utilized to study crack tip fields under large deformation [57, 58, 59, 60, 61, 62, 63]. Computational models can be used to validate the asymptotic solutions, to determine the region of validity for the crack tip fields, and to relate the amplitudes of these fields to external loadings and geometry.

The finite strain crack tip fields exhibit characteristics that are drastically different from those of the LEFM solution, which we intend to discuss in this review. These departures from LEFM offer important insights to the understanding of soft material fracture and can be an extremely useful tool aiding interpretation of experiments. We believe that the potentials of these theoretical results have not been fully exploited because of the lengthy and complicated analyses necessary to obtain finite strain solutions. In addition, the key ideas behind these results are not presented in forms readily accessible for non-specialist in nonlinear elasticity. To fill this gap, we review previous finite strain solutions for crack tip fields. Our aims are to provide 
physical insights into these complicated solutions, bridge the theoretical results with experimental data, and highlight pitfalls in applications that may lead to confusion or misunderstandings. To limit the length of this review, we focus on incompressible materials. This focus can be justified by noting that most soft elastomers and gels undergo isochoric deformation, at least within a certain time scale, and can be considered as incompressible.

The plan of this paper is as follows: in Section 2, we briefly summarize the background information for crack tip asymptotic analysis, including geometry, governing equations, boundary equations, and material models. We also listed the LEFM crack tip solutions for comparison. In Section 3, we summarize finite strain crack tip solutions in incompressible materials for different geometries: plane strain, plane stress, anti-plane shear and interface cracks. Discussions on the true stress fields in deformed configuration are made in Section 4. Section 5 presents results and discussions on J-integral. Summary and additional discussions are presented in Section 6.

\section{Geometry, Notations and Basic Equations}

\subsection{Governing Equations: Kinematics and Equilibrium}

We use a Cartesian coordinate system with orthonormal basis vectors $\vec{e}_{i}$ to specify the positions of material points in the elastic solid (see Figure 1). We choose the reference configuration to be the undeformed, stress-free elastic body and we identify a material point in this configuration by its position vector $\vec{X}=X_{i} \vec{e}_{i}$. In this work, we adopted the summation convention of summing over repeated indices. After deformation, this material point moves to $\vec{x}=x_{i}\left(X_{1}, X_{2}, X_{3}\right) \vec{e}_{i}$ with respect to the same coordinate system. The displacement of the material point $\vec{X}, \vec{u}=u_{i}\left(X_{1}, X_{2}, X_{3}\right) \vec{e}_{i}$, is

$$
u_{i}\left(X_{1}, X_{2}, X_{3}\right)=x_{i}\left(X_{1}, X_{2}, X_{3}\right)-X_{i}
$$

Since our focus is on planar deformations (plane strain, plane stress and anti-plane shear) where all field quantities are independent of $X_{3}$, we consider in-plane displacements

$$
u_{\alpha}\left(X_{1}, X_{2}\right)=x_{\alpha}\left(X_{1}, X_{2}\right)-X_{\alpha} \quad \alpha=1,2
$$

In this work, Greek indices range from 1 to 2. In the plane stress formulation (see [49]), $\left(X_{1}, X_{2}\right)$ denote the mid-plane coordinates of a material point in the undeformed reference 
configuration. Since the behavior of crack tip fields is independent of specimen geometry, the near tip analysis can be carried out for a semi-infinite crack occupying $X_{1}<0, X_{2}=0^{ \pm}$, with the undeformed crack tip at the origin: $X_{1}=X_{2}=0$. In finite deformation, the displacement of the crack tip $x_{\alpha}\left(X_{1}=0, X_{2}=0\right)$ cannot be neglected. Therefore, we introduce

$$
y_{\alpha}\left(X_{1}, X_{2}\right) \equiv x_{\alpha}\left(X_{1}, X_{2}\right)-x_{\alpha}\left(X_{1}=0, X_{2}=0\right),
$$

which measures the coordinates of deformed material points relative to the current crack tip position.

In both plane stress and plane strain problems, the equilibrium equations are:

$$
\begin{aligned}
& \frac{\partial \sigma_{\alpha \beta}}{\partial X_{\beta}}=0, \\
& \sigma_{\alpha v} F_{\beta v}=F_{\alpha v} \sigma_{\beta v},
\end{aligned}
$$

where $\sigma_{\alpha \beta} \vec{e}_{\alpha} \vec{e}_{\beta}$ is the nominal stress tensor, which is also known as the first Piola-Kirchhoff stress tensor, and

$$
F_{\beta v}=\frac{\partial x_{\beta}}{\partial X_{v}}=\delta_{\beta v}+\frac{\partial u_{\beta}}{\partial X_{v}}
$$

is the Cartesian components of the in-plane deformation gradient tensor. Note that the nominal stress tensor is generally non-symmetric, and (4b) is derived from the balance of angular momentum and is satisfied identically for any hyperelastic solids. For incompressible materials, the true in-plane stress $\tau_{\alpha \beta} \vec{e}_{\alpha} \vec{e}_{\beta}$ is symmetric and is related to the nominal stress tensor by

$$
\tau_{\alpha \beta}=\sigma_{\alpha v} F_{\beta v} .
$$

For anti-plane shear deformation, there is only one non-zero displacement $u_{3}=w\left(X_{1}, X_{2}\right)$. Knowles [47] derived that for a crack with traction free boundary conditions, the out of plane nominal stress components $\sigma_{3 \alpha}$ are equal to the true stress components $\tau_{3 \alpha}$, and satisfy the following equilibrium equation:

$$
\frac{\partial \sigma_{3 \alpha}}{\partial X_{\alpha}}=0 .
$$

The equilibrium equations (4a) and (6) must be supplemented by a constitutive model. In the section below, we summarize most of the constitutive models used in the analysis of crack tip fields. 


\subsection{Constitutive Models}

For an incompressible isotropic elastic solid, the strain energy density function $W=\Phi\left(I_{1}, I_{2}\right)$ is a function of two strain invariants

$$
I_{1}=C_{i i}=\lambda_{I}^{2}+\lambda_{I I}^{2}+\lambda_{I I I}^{2}, I_{2}=\frac{1}{2}\left(C_{i i} C_{i j}-C_{j i} C_{i j}\right)=\lambda_{I}^{2} \lambda_{I I}^{2}+\lambda_{I I}^{2} \lambda_{I I I}^{2}+\lambda_{I}^{2} \lambda_{I I I}^{2}
$$

where $C_{i j} \equiv F_{i p} F_{j p}$ is the right Cauchy-Green tensor and $\lambda_{I}, \lambda_{I I}, \lambda_{I I I}$ are the principal stretches. Since most of the analyses were carried out with strain energy density functions which depend only on $I_{1}$, we will restrict ourselves to this situation. However, it should be noted that, in plane strain deformation, $I_{1}=I_{2}$ so that strain energy density $W$ can be written as a function of $I_{1}$ only (see example below). In the following, we define $I \equiv I_{1}$.

In plane strain, the relation between the in-plane nominal stress/true stress components and deformation gradient is [39]:

$$
\sigma_{\alpha \beta}=2 \frac{d W}{d I} F_{\alpha \beta}-p F_{\beta \alpha}^{-1} \Leftrightarrow \tau_{\alpha \beta}=2 \frac{d W}{d I} F_{\alpha \nu} F_{\beta v}-p \delta_{\alpha \beta}
$$

where $p$ is a scalar pressure field that serves as a Lagrange multiplier to enforce the incompressibility constraint, that is,

$$
\operatorname{det}\left\lfloor F_{\alpha \beta}\right\rfloor=1 .
$$

It should be emphasized that $p$ is not equal to the true hydrostatic pressure defined as $-\tau_{\alpha \alpha} / 3$. Instead, using (7a) it can be shown that $p=-\tau_{\alpha \alpha} / 3+2(d W / d I) I / 3$. The only non-vanishing out of plane component of the nominal stress tensor is:

$$
\sigma_{33}=-p+2 \frac{d W}{d I}=\tau_{33} .
$$

In (7a), the strain energy density function $W$ is defined by $W(I)=\Phi\left(I_{1}=I, I_{2}=I\right)$.

In plane stress, the pressure field can be eliminated using the fact that the out of plane stresses are zero, resulting in [49]

$$
\sigma_{\alpha \beta}=2 \frac{d W}{d I}\left(F_{\alpha \beta}-\lambda_{I I I}^{2} F_{\beta \alpha}^{-1}\right) \Rightarrow \tau_{\alpha \beta}=2 \frac{d W}{d I}\left(F_{\alpha \nu} F_{\beta v}-\lambda_{I I I}^{2} \delta_{\alpha \beta}\right)
$$

where $\lambda_{I I}$ denotes the out of plane stretch ratio.

Finally, in anti-plane shear, the out of plane shear stress components are 


$$
\sigma_{3 \alpha}=2 \frac{d W}{d I} F_{3 \alpha}
$$

\subsubsection{The Mooney-Rivlin model}

For a Mooney-Rivlin solid we have:

$$
\Phi\left(I_{1}, I_{2}\right)=\beta_{1}\left(I_{1}-3\right)+\beta_{2}\left(I_{2}-3\right)
$$

where $\beta_{1}, \beta_{2}$ are material constants such that $2\left(\beta_{1}+\beta_{2}\right)=\mu$ where $\mu$ is the small strain shear modulus. In plane strain, $I_{1}=I_{2}=I$ so that

$$
W(I)=\Phi\left(I_{1}=I, I_{2}=I\right)=\left(\beta_{1}+\beta_{2}\right)(I-3) .
$$

For a neo-Hookean solid, $\beta_{1}=\mu / 2$ and $\beta_{2}=0$.

\subsubsection{The generalized neo-Hookean model}

A widely used strain energy density function is the Generalized neo-Hookean Model (GNM):

$$
W=\frac{\mu}{2 b}\left\{\left[1+\frac{b}{n}(I-3)\right]^{n}-1\right\},
$$

where $\mu$ is the small strain shear modulus, $b>0$ and $n>1 / 2$ are material parameters. In uniaxial tension, it has been shown that $b$ controls the extent of linearity and $n$ the degree of strain hardening [45]. The condition $n>1 / 2$ ensures that the elastostatic equilibrium equations are elliptic. In particular, Geubelle and Knauss [45] have shown that the condition $n<1 / 2$ leads to unstable behavior in uniaxial tension, i.e., the nominal tensile stress decreases as the stretch ratio increases.

\subsubsection{The exponentially hardening material model}

Another constitutive model which captures the strain hardening behavior of elastomers and gels is the exponentially hardening material. For example, Seitz et al [64] found that the experimental data of their uniaxial compression tests on acrylic triblock copolymers can be fitted very well using an exponentially hardening material model. This model was also used to fit cavitation experiments in polyurethane elastomers [19]. The strain energy density function of this model is 


$$
W=\frac{\mu J_{n}}{2}\left\lfloor\exp \left(\frac{I-3}{J_{n}}\right)-1\right\rfloor,
$$

where $J_{n}$ is a material constant that measures the degree of strain hardening. Larger $J_{n}$ implies less strain hardening. The exponentially hardening model captures the finite extensibility of gels and rubbers by rapidly increasing the strain energy once $I-3>J_{n}$. It is interesting to note that the exponential model is the formal limit of the GNH model as $n \rightarrow \infty$.

\subsubsection{The Gent model}

Finally, a model that captures the finite extensibility of polymer chains is the Gent model (GM) [65], the strain energy density function of GM is

$$
W=-\frac{\mu J_{n}}{2} \ln \left\lfloor 1-\frac{I-3}{J_{n}}\right\rfloor,
$$

where $J_{n}$ is a material parameter that controls the degree of strain hardening. In tension and biaxial tests, this model performs just as well as the more physically based Arruda-Boyce model $[66,67]$.

\subsubsection{Comparison of different material models}

For sufficiently small strains, that is, when $I-3$ is small in comparison to $n / b(\mathrm{GNH})$ or $J_{n}(\mathrm{EM}$ or Gent), all three models approach the neo-Hookean model (ideal rubber). In a uni-axial tensile test, the nominal stress $\sigma$ is related to the stretch ratio $\lambda$ by

$$
\sigma=\left.2 \frac{d W}{d I}\right|_{I=\lambda^{2}+2 \lambda^{-1}}\left[\lambda-\lambda^{-2}\right]
$$

The effect of strain hardening can be examined by plotting the reduced stress,

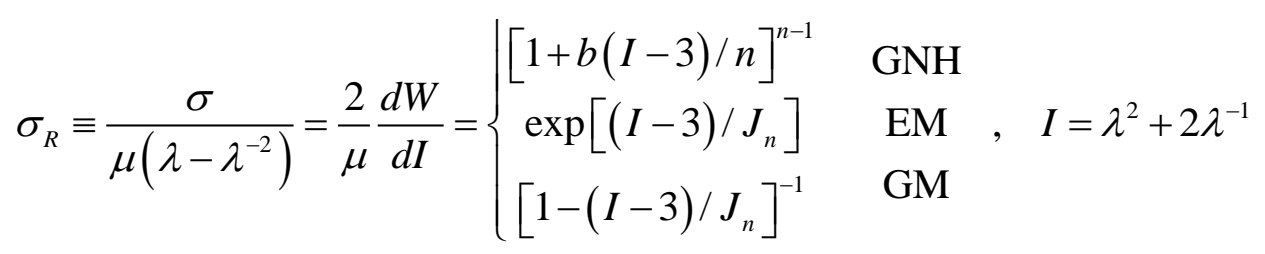

versus the stretch ratio, as shown in Figure 2. Note the reduced stress for a neo-Hookean solid is 1 by definition. 


\subsection{Boundary conditions}

The appropriate boundary conditions for local analysis is to require that the crack faces are free of traction. For the ease of asymptotic analysis, we will use polar coordinates $(r, \theta)$ at the undeformed crack tip, where $r \equiv \sqrt{X_{\alpha} X_{\alpha}}$ and $|\theta|<\pi$, to denote material points (see Figure 1a). For plane strain or stress, the traction free condition is

$$
\sigma_{22}(r>0, \theta= \pm \pi)=\sigma_{12}(r>0, \theta= \pm \pi)=0 \quad \text { (plane strain, plane stress). }
$$

The boundary conditions above are written in terms of the nominal stress in undeformed configuration. Assuming the deformation has sufficient regularity so that an arc on the boundary in the undeformed configuration is carried continuously by the deformation into an arc in the deformed configuration, then (18) is equivalent to the traction free condition in terms of the true stress in deformed configuration. However, after deformation the crack surface deforms to a new shape which is yet to be determined. Thus it is much more convenient to use nominal stress for the traction free boundary conditions. For anti-plane shear, the traction free condition is

$$
\sigma_{32}(r>0, \theta= \pm \pi)=0 \quad \text { (anti-plane shear). }
$$

\subsection{Crack Tip Fields in Linear Elastic Fracture Mechanics (LEFM)}

In the linearized theory of elasticity, the displacements and its gradients are assumed to be sufficiently small so differences between different stress measures (e.g. the nominal and true stress) can be neglected. Similarly, the spatial distributions of stresses are insensitive to whether one uses the reference coordinates $\left(X_{1}, X_{2}\right)$ or deformed coordinates $\left(y_{1}, y_{2}\right)$ as independent variables. Obviously, these assumptions break down near the crack tip where deformation is large. Nevertheless, it is useful to review the crack tip stress fields according to LEFM [68]. The linearized theory has the following features:

1. Stress fields can be superimposed, so the local stresses ahead of a smoothly varying crack front is a linear combination of plane strain Mode I (opening Mode) and plane strain Mode II (sliding Mode) and Mode III (Anti-plane shear) fields given below.

2. The stresses are separable: they are products of a power function of $r$ and a periodical function of $\theta$. Spatial distribution of the in-plane crack tip stress fields is independent of elastic moduli. 
3. In Mode I, II and III, every component of the stress tensor are unbounded with the same characteristic inverse square root singularity $1 / \sqrt{r}$ as the crack tip is approach $(r \rightarrow 0)$.

\subsubsection{Plane strain (Mode I)}

Mode I is defined by requiring the displacement fields to be symmetric about the crack line, i.e.,

$$
u_{1}\left(X_{1}, X_{2}\right)=u_{1}\left(X_{1},-X_{2}\right), u_{2}\left(X_{1}, X_{2}\right)=-u_{2}\left(X_{1},-X_{2}\right) .
$$

The in-plane stresses are

$$
\left(\begin{array}{l}
\sigma_{11} \\
\sigma_{22} \\
\sigma_{12}
\end{array}\right)=\frac{K_{I}}{\sqrt{2 \pi r}} \cos \frac{\theta}{2}\left\{\begin{array}{c}
1-\sin \frac{\theta}{2} \sin \frac{3 \theta}{2} \\
1+\sin \frac{\theta}{2} \sin \frac{3 \theta}{2} \\
\sin \frac{\theta}{2} \cos \frac{3 \theta}{2}
\end{array}\right\}+\text { higher order terms }
$$

In plane strain, the only non-zero out of plane stress component is $\sigma_{33}$. For an incompressible solid, it is

$$
\sigma_{33}=\frac{1}{2}\left(\sigma_{11}+\sigma_{22}\right)=\frac{K_{I}}{\sqrt{2 \pi r}} \cos \frac{\theta}{2}+\text { higher order terms . }
$$

In particular, for plane strain,

$$
\sigma_{11}=\sigma_{22}=\sigma_{33}=\frac{K_{I}}{\sqrt{2 \pi r}}, \quad \text { at } \theta=0 .
$$

Equation (21c) implies that material points directly ahead of the tip of a plane strain crack in an incompressible solid are under hydrostatic tension. The amplitude of the stress singularity is uniquely characterized by a single parameter $K_{I}$, the Mode $I$ stress intensity factor. The stress intensity factor cannot be determined by local analysis, since it is related to the applied load and the specimen geometry.

The near tip displacement fields for an incompressible solid are given by

$$
\left(\begin{array}{l}
u_{1} \\
u_{2}
\end{array}\right)=\frac{K_{I}}{\mu} \sqrt{\frac{r}{2 \pi}} \sin ^{2} \frac{\theta}{2}\left(\begin{array}{c}
\cos \frac{\theta}{2} \\
\sin \frac{\theta}{2}
\end{array}\right)+\text { higher order terms }
$$

where $\mu$ is the shear modulus. In particular, the crack tip opening displacement is 


$$
u_{c t o d} \equiv 2 u_{2}(r, \theta=\pi)=2 \frac{K_{I}}{\mu} \sqrt{\frac{r}{2 \pi}} .
$$

Since initially $X_{2}=0$ on crack surface,

$$
y_{1}(r, \theta= \pm \pi)=X_{1}, \quad y_{2}(r, \theta= \pm \pi)= \pm u_{2}(r, \theta=\pi)= \pm \frac{K_{I}}{\mu} \sqrt{\frac{-X_{1}}{2 \pi}} .
$$

This implies the local crack profile is in a parabolic shape, that is,

$$
y_{2}= \pm \frac{K_{I}}{\mu} \sqrt{\frac{-y_{1}}{2 \pi}}, \quad \text { as } y_{1} \rightarrow 0^{-} .
$$

\subsubsection{Plane strain (Mode II)}

In Mode II, the displacement is anti-symmetric about the crack line, that is

$$
u_{1}\left(X_{1}, X_{2}\right)=-u_{1}\left(X_{1},-X_{2}\right), u_{2}\left(X_{1}, X_{2}\right)=u_{2}\left(X_{1},-X_{2}\right) .
$$

The stress fields are given by

$$
\left(\begin{array}{l}
\sigma_{11} \\
\sigma_{22} \\
\sigma_{12}
\end{array}\right)=\frac{K_{I I}}{\sqrt{2 \pi r}}\left\{\begin{array}{c}
-\sin \frac{\theta}{2}\left(2+\cos \frac{\theta}{2} \cos \frac{3 \theta}{2}\right) \\
\sin \frac{\theta}{2} \cos \frac{\theta}{2} \cos \frac{3 \theta}{2} \\
\cos \frac{\theta}{2}\left(1-\sin \frac{\theta}{2} \sin \frac{3 \theta}{2}\right)
\end{array}\right\}+\text { higher order terms }
$$

where $K_{I I}$ is the Mode II stress intensity factor. For an incompressible material, the only nonvanishing out of plane stress component is

$$
\sigma_{33}=\frac{1}{2}\left(\sigma_{11}+\sigma_{22}\right)=-\frac{K_{I I}}{\sqrt{2 \pi r}} \sin \frac{\theta}{2} .
$$

The displacement fields for an incompressible material are:

$$
\left(\begin{array}{l}
u_{1} \\
u_{2}
\end{array}\right)=\frac{K_{I I}}{\mu} \sqrt{\frac{r}{2 \pi}} \sin \frac{\theta}{2}\left(\begin{array}{c}
1+\cos ^{2} \frac{\theta}{2} \\
\cos \frac{\theta}{2} \sin \frac{\theta}{2}
\end{array}\right)
$$

In Mode II, the relative displacement of the crack faces are of pure shear with opening displacement $u_{2}(r, \theta= \pm \pi)$ exactly zero.

\subsubsection{Plane Stress}

For thin specimens, e.g. a plate with thickness much smaller than all other relevant geometrical dimensions, it is common to use plane stress theory to compute the crack tip fields. 
In this theory, the out of plane stresses $\sigma_{3 i}$ are taken to be zero, and the out of plane variation of the in-plane displacement and stress fields are averaged in the thickness direction. For small strains, it turns out that the in-plane near tip stresses for plane stress Mode I and Mode II cracks are given also by (21a) and (26a). The displacement fields also have the same square root dependence on the distance from the crack tip, but the angular distributions of these fields should be modified to:

$$
\begin{aligned}
& \left(\begin{array}{l}
u_{1} \\
u_{2}
\end{array}\right)=\frac{K_{I}}{\mu} \sqrt{\frac{r}{2 \pi}}\left(\begin{array}{c}
\cos \frac{\theta}{2} \\
\sin \frac{\theta}{2}
\end{array}\right)\left(\frac{4}{3}-\cos ^{2} \frac{\theta}{2}\right) \quad \text { Mode I, } \\
& \left(\begin{array}{l}
u_{1} \\
u_{2}
\end{array}\right)=\frac{K_{I I}}{\mu} \sqrt{\frac{r}{2 \pi}}\left(\begin{array}{c}
\sin \frac{\theta}{2}\left(\frac{4}{3}+\cos ^{2} \frac{\theta}{2}\right) \\
\cos \frac{\theta}{2}\left(-\frac{1}{3}+\sin ^{2} \frac{\theta}{2}\right)
\end{array}\right) . \quad \text { Mode II. }
\end{aligned}
$$

Thus, in Mode I, the local crack opening profile in plane stress is:

$$
y_{2}= \pm \frac{4 K_{I}}{3 \mu} \sqrt{\frac{-y_{1}}{2 \pi}}, \quad y_{1} \rightarrow 0^{-} .
$$

\subsubsection{Mode III (Anti-plane shear)}

In Mode III, the only non-vanishing displacement is the out of plane component $u_{3}$ which is assumed to depend only on in the in-plane coordinates $X_{\alpha}$. As a result, all stress components vanish except $\sigma_{3 \alpha}=\sigma_{\alpha 3}$. Near the crack tip, they are:

$$
\left(\begin{array}{l}
\sigma_{31} \\
\sigma_{32}
\end{array}\right)=\frac{K_{I I I}}{\sqrt{2 \pi r}}\left\{\begin{array}{c}
-\sin \frac{\theta}{2} \\
\cos \frac{\theta}{2}
\end{array}\right\}+\text { higher order terms, }
$$

where $K_{I I I}$ is the Mode III stress intensity factor. The out of plane displacement field near the crack tip is

$$
u_{3}=\frac{K_{I I I}}{\mu} \sqrt{\frac{2 r}{\pi}} \sin \frac{\theta}{2}+\text { higher order terms } .
$$

\section{Crack Tip Fields under Large Deformation}


The goal of this section is to provide an accessible summary of the theoretical results of finite strain crack-tip deformation and stress fields in incompressible elastic solids. We divide our presentation into five parts: a brief description of asymptotic analysis techniques used (Section 3.1), summaries of solutions for cracks in homogeneous solids under plane strain (Section 3.2), plane stress (Section 3.3) and anti-plane shear (Section 3.4) conditions, as well as a short discussion on interface cracks (Section 3.5).

\subsection{Method of Asymptotic Analysis}

In their pioneering work, Knowles and Sternberg [31] performed the crack-tip asymptotic analysis using a series expansion of the deformed coordinates, similar to the idea behind William's expansion in LEFM. Specifically, the deformed coordinates $y_{1}$ and $y_{2}$ were assumed to be a series consisting of separable functions of the polar coordinates $(r, \theta)$ in the undeformed material coordinates (see Figure 1) in the vicinity of the crack tip where $r \rightarrow 0$ :

$$
y_{\alpha}(r, \theta)=r^{m_{\alpha}} v_{\alpha}(\theta)+r^{p_{\alpha}} q_{\alpha}(\theta)+\ldots, \quad m_{\alpha}<p_{\alpha}, \alpha=1,2
$$

where $m_{\alpha}, p_{\alpha}$ are unknown exponents, and $v_{\alpha}(\theta), q_{\alpha}(\theta)$ are unknown functions describing the angular variation. Substituting (33) into the constitutive model to obtain the nominal stress components, then substituting these stresses into the equilibrium equation $(4 a, b)$ and boundary condition (18), and retaining the most dominating terms yielded an eigenvalue problem for $v_{\alpha}(\theta)$ and $m_{\alpha}$. In principle all the higher order terms can also be obtained by repeating this procedure [32]. This method has been adopted in many subsequent analyses $[39,45,49,52]$ and is the most widely used method in the literature.

The series expansion method, although straightforward to implement, does pose restrictions on the functional form of the near-tip solutions. Because each term in (33) are separable, it does not always work for material models that do not exhibit power-law stiffening behavior. Indeed, even with the assumption of power-law stiffening, it is possible that the assumed form in (33) does not admit any solutions (e.g. $n=3 / 2$ in plane strain [39]). This difficulty can be avoided using a method based on hodograph transform [46]. The hodograph transform, introduced by Neuber [69], can convert the nonlinear partial differential equation (PDE) for the anti-plane shear crack derived from (6), into a linear PDE [47] . A detailed discussion of hodograph transform can be found in Rice [70]. Compared with the series expansion method, this method does not made a-priori assumption about the analytical form of 
the near tip field and thus can accommodate more complicated material models (see Section 3.3 for example).

Finally, we note that Bouchbinder et al. [26, 27, 28] obtained a weakly nonlinear crack tip field by perturbing the LEFM crack tip solutions for moderate deformations. Their weakly nonlinear theory can nicely explain the significant deviation between LEFM crack tip solutions and experimental data for quasi-static and dynamic cracks in brittle polyacrylamide gels [24, 25]. In particular, the experimental data in Liven et al. [25] suggested that there existed a length scale around the crack tip within which the LEFM solution broke down and the fully nonlinear crack tip solutions based on large deformation theory prevailed. The weakly nonlinear solution was shown to well describe how the LEFM field bridges with the fully nonlinear field as the crack tip was approached. Recently an extension of the weakly nonlinear theory was developed where perturbation was performed with respect to a highly deformed state [71]. The new theory was found to capture experimentally measured crack tip deformation fields under uni-axial prestretch ranging from 1.058 to 1.129 . In comparison to the fully nonlinear solution, the weakly nonlinear solution is only valid in an intermediate asymptotic region and requires the assumption that large deformation effects are confined to a small region near the crack tip, similar to the small scale yielding assumption in elastic-plastic fracture. However, since the weakly nonlinear solution is independent of nonlinear material models, it may be advantageous for developing a nonlinear crack-tip enrichment function to be used in the extended finite element method (XFEM) for a wide class of nonlinear elastic material models [61, 72]. Given that our focus is on the fully nonlinear theory of finite strain elastostatics, an in-depth review of the weakly nonlinear solution is not performed here.

\subsection{Plane strain crack in homogeneous materials}

Many of the key results are due to Stephenson [39]. Instead of using a specific constitutive model, he assumed that near the crack tip (where $I$ is expected to be unbounded), the elastic potential is governed by

$$
W(I>>1)=A I^{n}+B I^{n-1}+o\left(I^{n-1}\right)
$$

where $A, B$ and $n>1 / 2$ are material constants with dimension of stress. The symbol $o$ in (34)

indicates that the next term in the expansion is asymptotically small in comparison with $I^{n-1}$ as the crack tip is approached. Note that the special case of $n=1$ corresponds exactly (for all $I$ ) to a 
neo-Hookean or a Mooney-Rivlin material, where $A=\mu / 2, B=-3 \mu / 2$, and $\mu$ is the small strain shear modulus. For the GNH model, $A=(\mu / 2) b^{n-1} n^{-n}$.

\subsubsection{Existence of Mode II}

As noted in Section 2.5, in linear elasticity theory, the crack can be in a state of pure Mode I or pure Mode II. These two modes are independent, in the sense that the near tip stress field due to any combination of in-plane loading can be written as a linear combination of them. In large deformation theory, Stephenson [39] has shown that at least for the Mooney-Rivlin model $(n=1)$ pure Mode II does not exist, that is, there is no displacement field that satisfies the anti-symmetric condition in (25). For the more general model with $n \neq 1$, Stephenson [39] has shown that the crack displacement profiles must have nonzero openings irrespective of the manner of the far field loading. In other words, the crack surface can open even if only shear stresses are applied in the far field. This has been recognized and explicitly demonstrated in a recent work by Harpz and Bouchbinder [73]. The absence of a pure Mode II solution and the breakdown of superposition principle in large deformation bring difficulty for the search for a general mix-mode crack tip solution In Sections 3.2.2-3.2.4, we will first discuss the Mode-I solution where the crack tip field obeys the symmetry condition in (20). The mix-mode solution will be discussed in Section 3.2.5.

\subsubsection{Crack tip deformation field (Mode I)}

The characteristics of the deformation has a transition at $n=3 / 2$. The deformed coordinates near the tip of a Mode I crack, as $r \equiv \sqrt{X_{\alpha} X_{\alpha}} \rightarrow 0$ are:

$$
\begin{aligned}
& y_{1}=\left\{\begin{array}{lc}
-b_{0} r^{2-\frac{1}{n}}[U(\theta, n)]^{2}, & 1 / 2<n<3 / 2 \\
-\frac{1}{a} r^{1+\frac{1}{2 n}} H(\theta, n), & n>3 / 2
\end{array},\right. \\
& y_{2}=a r^{1-\frac{1}{2 n}} U(\theta, n),
\end{aligned}
$$

where $a$ and $b_{0}$ are unknown positive amplitudes that depends on the applied loading and specimen geometry. An example of how these parameters are related to external loading and specimen geometry is given in Section 4.2. In addition, 
$U(\theta, n)=\sin (\theta / 2) \sqrt{1-\frac{2 \kappa^{2} \cos ^{2}(\theta / 2)}{1+\omega(\theta, n)}}[\omega(\theta, n)+\kappa \cos \theta]^{\kappa / 2}, \quad 0 \leq|\theta| \leq \pi$,

$\kappa=1-\frac{1}{n}$,

$\omega(\theta, n)=\sqrt{1-\kappa^{2} \sin ^{2} \theta}$.

The angular function $H(\theta, n)$ in $y_{1}$ when $n>3 / 2$ is given by

$H(\theta, n)=-\frac{n^{5 / 2}}{m^{2}}[\omega(\theta, n)+\kappa \cos \theta]^{2-m}\left\lfloor\frac{m}{2-m} F\left(\frac{1}{2}-\frac{1}{m}, \frac{1}{2} ; \frac{3}{2}-\frac{1}{m} ; \cos ^{2} \xi_{0}\right)-\kappa \sin \xi_{0}\right\rfloor$,

where

$$
\begin{aligned}
& m=1-\frac{1}{2 n}, \\
& \cos \xi_{0}=\frac{1}{n \sqrt{2}} \frac{\sqrt{1+\kappa \sin ^{2} \theta-\omega(\theta, n) \cos \theta}}{\omega(\theta, n)+\kappa \cos \theta}, 0 \leq \xi_{0} \leq \frac{\pi}{2} .
\end{aligned}
$$

In (37a), $F$ is the hypergeometric function. The functions $U$ and $H$ are plotted in Figures 3a,b for different values of $n$. Note that symmetry condition (20) implies that $y_{1}$ and $y_{2}$ should be even and odd functions of $\theta$, respectively. This is satisfied by the solutions listed above. Specifically for $y_{2}, U(\theta, n)$ is an odd function of $\theta$ with the following properties:

$$
\begin{aligned}
& U(\theta=0, n)=0, \\
& U(\theta= \pm \pi, n)= \pm n^{\frac{1-n}{2 n}} .
\end{aligned}
$$

For $y_{1}$ with $1 / 2<n<3 / 2,[U(\theta, n)]^{2}$ is an even function of $\theta$. For $y_{1}$ with $n>3 / 2, H(\theta, n)$ is an even function of $\theta$ with the following properties:

$$
\begin{aligned}
& H(\theta=0, n)=-\frac{4 n^{9 / 2}}{(2 n-1)^{2}}\left[2-\frac{1}{n}\right]^{1+\frac{1}{2 n}} \frac{1}{n(2 n+1)}, \\
& H(\theta= \pm \pi, n)=-\frac{4 n^{9 / 2}}{(2 n-1)^{2}} n^{-1-\frac{1}{2 n}}\left(\frac{2 n-1}{2 n+1}\right) \frac{\sqrt{\pi} \Gamma\left(\frac{2 n-3}{2(2 n-1)}\right)}{\Gamma\left(\frac{-1}{2 n-1}\right)},
\end{aligned}
$$

where $\Gamma$ is the Gamma function. Note $\Gamma(-1 /(2 n-1))<0$ for $n>3 / 2$. 
Equations $(35 \mathrm{a}, \mathrm{c})$ show that the near tip displacement fields of materials that exhibits small or moderate strain hardening, i.e., $1 / 2<n<3 / 2$, depends on two independent parameters $a, b_{0}$ whereas in small strain theory, it depends only on the Mode I stress intensity factor. However, as the strain hardening parameter $n$ increases $(n>3 / 2)$, the displacement field depends on a single parameter $a$ (see (35b)). In Sections 3.2.3 and 4.1, we show that $a$ is associated with the singular stress components and the J-integral (see (43a,b), (46c) and (89a)). The transition case of $n=3 / 2$ was also solved by Stephenson [39] but is not reviewed here because in practical applications it highly unlikely that $n$ is exactly $3 / 2$ when one fits the model behavior in (34) to experimental data.

The near tip crack opening profiles in Mode I can be evaluated by setting $\theta= \pm \pi$ in (35a,b) and applying (38b) and (39b). For $1 / 2<n<3 / 2$, the crack opening profile is:

$$
y_{2}= \pm a \sqrt{\frac{y_{1}}{-b_{0}}} \text { at } \theta= \pm \pi \text { for } 1 / 2<n<3 / 2
$$

where $a>0$ and $b_{0}>0$ since $y_{2}(\theta=\pi, n)>0$ and $y_{1}(\theta=\pi, n)<0$ (see Figure 1b). Thus, the crack has the parabolic shape for all $1 / 2<n<3 / 2$. Recall the case $n=1$ corresponds to a neoHookean or Mooney-Rivlin solid, albeit with different shear modulus. In contrast to LEFM, two independent parameters $a$ and $b_{0}$ are needed to specify the crack opening profile. For $n>3 / 2$, we have

$$
y_{2}= \pm a n^{\frac{1-n}{2 n}}\left[\frac{-a y_{1}}{H(\theta=\pi, n)}\right]^{\frac{2 n-1}{2 n+1}}, \quad \text { at } \theta= \pm \pi \text { for } n>3 / 2 \text {. }
$$

where $H(\theta=\pi, n)$ is always positive for all $n>3 / 2$ (see (39b)). Equation (40b) shows that as the hardening parameter $n$ increases, the crack opening profile becomes more wedge-like. For large $n$, the exponent in $(40 \mathrm{~b}),(2 n-1) /(2 n+1)$, approaches 1 , and

$$
y_{2} \approx \pm \frac{2 a^{2}}{n \pi}\left|y_{1}\right|
$$

where we have used $H(\theta=\pi, n>>1) \simeq n^{1 / 2} \pi / 2$ (see (39b)). Thus, with sufficient strain hardening, the crack opening profile is determined by the single parameter $a$ in (35b).

\subsubsection{Crack tip stress fields}


Stephenson [39] derived the near tip behavior of the true stress $\tau_{\alpha \beta}$, but did not explicitly give results for the nominal stress $\sigma_{\alpha \beta}$. Since the nominal stress is needed to evaluate the energy release rate, here we first provide the crack tip behavior of $\sigma_{\alpha \beta}$ and then summarize the results of $\tau_{\alpha \beta}$.

Using (7a) and (35a,b), the leading order behavior of $\sigma_{2 \alpha}$ for $n>1 / 2$ was found to be

$$
\begin{array}{ll}
\sigma_{21} & =2 A n a^{2 n-1} r^{-1+\frac{1}{2 n}}\left[\hat{I}_{n}(\theta)\right]^{n-1} \eta_{1}(\theta, n), \\
\sigma_{22} & =2 A n a^{2 n-1} r^{-1+\frac{1}{2 n}}\left[\hat{I}_{n}(\theta)\right]^{n-1} \eta_{2}(\theta, n), \quad n>1 / 2
\end{array}
$$

where $A$ is the material constant defined in (34), $a$ is the constant coefficient in (35b), and

$$
\begin{aligned}
& \hat{I}_{n}(\theta)=\frac{(2 n-1)^{2}}{4 n^{3}}[\omega(\theta, n)+\kappa \cos \theta]^{-1 / n}, \\
& \eta_{1}(\theta, n)=\left(1-\frac{1}{2 n}\right) U(\theta, n) \cos \theta-U^{\prime}(\theta, n) \sin \theta, \\
& \eta_{2}(\theta, n)=\left(1-\frac{1}{2 n}\right) U(\theta, n) \sin \theta+U^{\prime}(\theta, n) \cos \theta .
\end{aligned}
$$

Note that $\kappa$ and $\omega(\theta, n)$ are defined in (36b,c), $U(\theta, n)$ is defined in (36a), and $U^{\prime}(\theta, n)$ is its derivative with respect to $\theta$, i.e.,

$$
U^{\prime}(\theta, n)=\frac{\sqrt{2 n-1}}{2 \sqrt{2} n} \sqrt{\omega+\cos \theta}(\omega+\kappa \cos \theta)^{\kappa / 2} .
$$

When deriving the above results, we have used the following asymptotic solution of the pressure field $p$ (see (7a)) by Stephenson [39]:

$$
\begin{array}{cc}
p=r^{\frac{1}{2 n}} P(\theta, n)=-r^{\frac{1}{2 n}} 4 A b_{0} a^{2 n-3} n^{\frac{5}{2}-n}\left(1-\frac{1}{2 n}\right)^{2 n-2}[\omega(\theta, n)+\kappa \cos \theta]^{1 / 2 n} \sqrt{1-\cos ^{2} \xi_{0}} & \text { for } 1 / 2<n<3 / 2 \\
p=o\left(r^{-1+1 / n}\right) & \text { for } n>3 / 2 .
\end{array}
$$

In (44a), $b_{0}$ is the constant coefficient in (35a) and $\cos \xi_{0}$ is defined in (37c). The leading order terms of the other two nominal stress components, $\sigma_{1 \alpha}$, depends on $n$. For $1 / 2<n<3 / 2$, we find $\sigma_{11}=-4 A n a^{2 n-2} b_{0}\left[\hat{I}_{n}(\theta)\right]^{n-1} U(\theta, n) \eta_{1}(\theta, n)-a P(\theta, n) \eta_{2}(\theta, n)$, 
$\sigma_{12}=-4 A n a^{2 n-2} b_{0}\left[\hat{I}_{n}(\theta)\right]^{n-1} U(\theta, n) \eta_{2}(\theta, n)+a P(\theta, n) \eta_{1}(\theta, n), 1 / 2<n<3 / 2$.

Thus, these two components $\left(\sigma_{1 \alpha}\right)$ are bounded at the crack tip, while the other two $\left(\sigma_{2 \alpha}\right)$ has the same singularity. The amplitude of these singular terms are specified by a single parameter $a^{2 n-1}$ which is analogous to the stress intensity factor in LEFM. For $n>3 / 2$, since the asymptotic solution of the pressure field $p$ is not explicitly solved (see (44b)), it is not possible to determine a closed form asymptotic solution for $\sigma_{1 \alpha}$.

Next we summarize the near tip solutions of true stress components $\tau_{\alpha \beta}$ given in Stephenson [39]. Even though $\tau_{\alpha \beta}$ represent stress components in the deformed configuration, the leading order results were expressed in terms of the undeformed coordinates $(r, \theta)$ :

$$
\begin{aligned}
& \tau_{11}=\left\{\begin{array}{lr}
r^{1-\frac{1}{n}} 8 A a^{2 n-2} b_{0}^{2} n\left[\hat{I}_{n}(\theta)\right]^{n}[U(\theta, n)]^{2} & 1 / 2<n<3 / 2 \\
o\left(r^{-1+\frac{1}{n}}\right) & n>3 / 2
\end{array}\right. \\
& \tau_{12}=\left\{\begin{array}{lr}
-r^{-\frac{1}{2 n}} 4 A a^{2 n-1} b_{0} n\left[\hat{I}_{n}(\theta)\right]^{n} U(\theta, n) & 1 / 2<n<3 / 2 \\
r^{-1+\frac{1}{n}} \hat{T}_{12}(\theta, n) & n>3 / 2
\end{array},\right. \\
& \tau_{22}=r^{-1} 2 A a^{2 n}\left(1-\frac{1}{2 n}\right)^{2 n} n^{1-n}[\omega(\theta, n)+\kappa \cos \theta]^{-1} \text { for all } n>1 / 2 \text {. }
\end{aligned}
$$

In (46b), $\hat{T}_{12}(\theta, n)$ is an unknown function describing the angular variation of $\tau_{12}$ and is not solved by Stephenson [39]. In addition, one can show the following behavior for the out of plane normal stress $\tau_{33}$

$$
\tau_{33} \approx 2 A n a^{2 n-2}\left[\hat{I}_{n}(\theta)\right]^{n-1} r^{-1+\frac{1}{n}} \quad \text { for all } n>1 / 2 .
$$

Similar to the nominal stress, the amplitude of the dominant singular stress component $\tau_{22}$ is completely determined by $a$. Note that $\tau_{11} / \tau_{22}, \tau_{21} / \tau_{22}$ and $\tau_{33} / \tau_{22}$ all approach 0 as $r \rightarrow 0$. Thus the stress state ahead of the crack tip is, to first order, under pure uniaxial tension. Recall in LEFM (see (21c)), the region directly ahead of the crack tip is under pure hydrostatic tension in plane strain. If an ideal rubber model $(n=1)$ is used, then the region of high triaxiality is lost due to "crack blunting" under large deformation. As indicated by (46a-c) and (47), for very large 
$n$, i.e., materials with high strain stiffening effects, the difference between the singularity exponents of $\tau_{11}, \tau_{22}$ and $\tau_{33}$ becomes insignificant (all approaches -1), and the crack tip recovers its state of high triaxiality seen in LEFM. Also, as $n$ becomes very large, the crack opening profile changes parabolic to wedge-like (see Figure 4b), suggesting the increase in triaxiality is associated with re-sharpening of the crack faces. Finally, it is important to note that the true stresses in (46a-c) and (47) are expressed in terms of the undeformed coordinates $(r, \theta)$. To interpret experimental results, it is useful to express the true stresses in the deformed coordinate system $\left(y_{1}, y_{2}\right)$. This is an important issue which we shall address later in Section 5.1.

\subsubsection{Special case: $\mathbf{n}=1$ for neo-Hookean or Mooney Rivlin solid}

The solution for the special case of $n=1$ is particularly simple. In this case the material model in (34) reduces to a neo-Hookean model which is mathematically equivalent to a Mooney Rivlin model under plane strain. For this case $A=\mu / 2$ where $\mu$ is the small strain shear modulus. From $(35 \mathrm{a}, \mathrm{b})$, the asymptotic solutions for the deformed coordinates $\left(y_{1}, y_{2}\right)$ under Mode I condition are

$$
y_{1}=-b_{0} r \sin ^{2}(\theta / 2), \quad y_{2}=a r^{1 / 2} \sin (\theta / 2) .
$$

Using (41)-(45), the leading order terms for the nominal stress components are

$$
\sigma_{11}=\mu b_{0}, \sigma_{12}=o(1), \sigma_{21}=-\frac{\mu a}{2} r^{-1 / 2} \sin (\theta / 2), \sigma_{21}=\frac{\mu a}{2} r^{-1 / 2} \cos (\theta / 2) .
$$

Using (46), the leading order terms for the true stress components are:

$$
\tau_{11}=\mu b_{0}^{2} \sin ^{2}(\theta / 2), \tau_{12}=\tau_{21}=-\frac{\mu}{2} a b_{0} r^{-1 / 2} \sin (\theta / 2), \tau_{22}=\frac{\mu}{4} a^{2} r^{-1}
$$

Using (44a), the pressure field $p$ is found to be

$$
p=-\frac{2 \mu b_{0} \sqrt{r}}{a} \cos (\theta / 2)+O(r) \text {. }
$$

\subsubsection{Mixed-mode loading}

A schematic of crack deformation under mix-mode plane strain loading is shown in Figure 5. Stephenson's solution [39] of the mix-mode crack tip field was based on an important observation. Let $\left[Q_{\alpha \beta}\right]$ be the rotation matrix representing an arbitrary proper orthogonal tensor 
$Q_{\alpha \beta} \vec{e}_{\alpha} \vec{e}_{\beta}$. Assuming $y_{\beta}$ is a solution of the crack tip deformation, one can apply the rotation represented by $Q_{\alpha \beta}$ to the entire field $y_{\beta}$ such that

$$
y_{\alpha}^{*}=Q_{\alpha \beta} y_{\beta}, F_{\alpha V}^{*}=Q_{\alpha \beta} F_{\beta V} .
$$

The invariant $I$ (or $I_{1}$ defined in (6)), however, is not affected by this rotation. Also, the scalar pressure field $p$ in (7a) remain unchanged: $p^{*}=p$. Therefore, the new stress components due to this rotation are

$$
\sigma_{\alpha v}^{*}=Q_{\alpha \beta} \sigma_{\beta v}, \tau_{\alpha v}^{*}=Q_{\alpha \gamma} \tau_{\gamma \beta} Q_{v \beta}
$$

Since $Q_{\alpha \beta}$ is spatially constant, one can readily show that the new deformation field $y_{\alpha}^{*}$ also satisfies the governing equations in $(4 a, b)$ and $(7 b)$ and the traction free boundary conditions in (18), and thus is also a crack tip solution.

Based on the observation above, Stephenson [39] argued that in general one could assume $y_{1}$ and $y_{2}$ to possess similar first order asymptotic term under mixed-mode loading, that is,

$$
y_{1}=a_{1} r^{1-\frac{1}{2 n}} U(\theta, n)+o\left(r^{1-\frac{1}{2 n}}\right), y_{2}=a_{2} r^{1-\frac{1}{2 n}} U(\theta, n)+o\left(r^{1-\frac{1}{2 n}}\right),
$$

where $a_{1}$ and $a_{2}$ are constant amplitudes and $U(\theta, n)$ is given in (36a). Equation (54) is the same as the leading order behavior of $y_{2}$ under Mode-I loading (see (35b)). Stephenson [39] also derived higher order terms for $y_{1}$ and $y_{2}$. His results were expressed in terms of a canonical form $y_{1}^{*}$ and $y_{2}^{*}$ defined below

$$
y_{\alpha}^{*}=Q_{\alpha \beta} y_{\beta}, \quad \text { where }\left[Q_{\alpha \beta}\right]=\frac{1}{a}\left|\begin{array}{cc}
a_{2} & -a_{1} \\
a_{1} & a_{2}
\end{array}\right| \text { and } a=\sqrt{a_{1}^{2}+a_{2}^{2}} \text {. }
$$

The mix-mode solution in terms of $y_{1}^{*}$ and $y_{2}^{*}$ is

$$
y_{1}^{*}=\left\{\begin{array}{lc}
-b_{0} r^{2-\frac{1}{n}}[U(\theta, n)]^{2}+o\left(r^{2-\frac{1}{n}}\right), & 1 / 2<n<3 / 2 \\
-\frac{1}{a} r^{1+\frac{1}{2 n}} H(\theta, n)+o\left(r^{1+\frac{1}{2 n}}\right), & n>3 / 2
\end{array},\right.
$$




$$
y_{2}^{*}=\left\{\begin{array}{cc}
a r^{1-\frac{1}{2 n}} U(\theta, n)+\frac{1}{a} r^{m^{\prime}} \chi(\theta, n)+o\left(r^{m^{\prime}}\right), & 1 / 2<n<7 / 2 \\
a r^{1-\frac{1}{2 n}} U(\theta, n)+\frac{1}{a} r^{1+\frac{1}{2 n}} \chi(\theta, n)+o\left(r^{1+\frac{1}{2 n}}\right), & n \geq 7 / 2
\end{array},\right.
$$

where

$$
m^{\prime}=\frac{\kappa+\sqrt{\kappa^{2}+(8-4 / n) / n}}{2}>1-\frac{1}{2 n}, \quad \kappa=1-\frac{1}{n}
$$

and

$$
\begin{aligned}
\chi(\theta, n)= & -c_{1} \frac{B \kappa \sqrt{2 n}}{4 A(1-1 / 2 n)^{2}(1+n)}[\omega(\theta, n)+\kappa \cos \theta]^{m^{\prime}-1} \frac{(1+\kappa) \sin \theta}{\left(1+\kappa \sin ^{2} \theta+\omega(\theta, n) \cos \theta\right)^{1 / 2}} \\
& +c_{2}[\omega(\theta, n)+\kappa \cos \theta]^{m^{\prime}}\left\{\frac{1+\kappa \sin ^{2} \theta-\omega(\theta, n) \cos \theta}{[\omega(\theta, n)+\kappa \cos \theta]^{2} n^{2}}-1\right\}
\end{aligned}
$$

The constant coefficient $c_{1}$ is equal to 0 for $1 / 2<n<7 / 2$ and 1 for $n \geq 7 / 2$, while $c_{2}$ is undetermined for $1 / 2<n \leq 7 / 2$ and 0 for $n>7 / 2$. Recall that $A$ and $B$ are material constants defined in (34), and $\kappa$ and $\omega(\theta, n)$ are defined in $(36 \mathrm{~b}, \mathrm{c})$. It is interesting to note that the leading order term of $y_{1}^{*}$ in (56a) is the same as that of the Mode-I solution in (35a). Based on (55) and the solutions of $y_{1}^{*}$ and $y_{2}^{*}$ given in $(56 \mathrm{a}, \mathrm{b})$, one can obtain the first three asymptotic terms of $y_{1}$ and $y_{2}$ under any mix-mode loadings. For example one can obtain the Mode-I solution in (35a,b) by setting $a_{1}=0$. Note that in this case, the coefficient $c_{2}$ in (57b) must be 0 for all $n>1 / 2$ so that $y_{2}$ is an odd function of $\theta$.

It can be shown that the canonical stress fields $\sigma_{\alpha v}^{*}$ or $\tau_{\alpha v}^{*}$ are similar to those of the Mode I stress fields given in Section 3.2.3. Thus one can determine the general form of mixed-mode crack tip stress fields using the Mode-I fields and (53), with $Q_{\alpha \beta}$ defined by (55). Note that in particular, for $n>3 / 2$, the leading order behavior of the canonical fields is completely determined by the single parameter $a$.

\subsection{Plane stress crack in homogeneous materials}


The crack tip field for a plane stress crack undergoing large deformation was first determined by Wong and Shield [30], who studied the problem of a finite crack in a bi-axially loaded infinite sheet of incompressible neo-Hookean solid. Geubelle and Knauss [45] later analyzed the crack tip field in a generalized neo-Hookean solid (see (13)). However, their Mode-I solution is valid only for strain hardening exponent $n<n^{*} \approx 1.46$ (recall in plane strain, there is a transition of behavior at $n=3 / 2$ ), and hence their method can only address very moderate amount of strain hardening. This difficulty was removed by a later analysis of Long et al. [46], who presented a leading order solution for the Mode-I crack tip field that is valid for all $n>1 / 2$. They also extended the analysis to a non-power law hardening model, i.e., the exponentially hardening solid (see (14)). Here we summarize the Mode-I crack tip solution for GNH and EM solids. A brief discussion of the mixed-mode solution for GNH solids is provided at the end of Section. 3.3.1.

\subsubsection{Crack tip deformation field for GNH solids}

The leading order terms of Mode I deformation field $y_{1}$ and $y_{2}$ under plane stress condition are summarized below. Note that we have used slightly different notations than those in Geubelle and Knauss [45] and Long et al. [46] to be consistent with the notations in Section 3.2 .

$$
\begin{aligned}
& y_{1}=\left\{\begin{array}{ll}
c r^{d} g(\theta, n), \quad d<1+\frac{1}{4 n} & n<n^{*} \\
a^{-1 / 2} n^{(n-1) / 4 n} r^{1+\frac{1}{4 n}} g(\theta, n) & n>n^{*}
\end{array},\right. \\
& y_{2}=\operatorname{ar}^{1-\frac{1}{2 n}} U(\theta, n) \quad \text { for any } n>1 / 2 \text {. }
\end{aligned}
$$

where $a$ and $c$ are positive independent amplitudes that can only be determined by far field boundary conditions such as the specimen geometry and the applied loading. For $n<n^{*} \approx 1.46$,

the singularity exponent $d$ of $y_{1}$ and $g(\theta, n)$ are obtained numerically by solving a two-point boundary value problem [45], which is not detailed here. For this case, Geubelle and Knauss [45] obtained an approximate solution of $d$ :

$$
d=\frac{1}{2}\left\lfloor\kappa+\sqrt{\kappa^{2}+\left(1+\sqrt{1-\frac{\kappa^{2}}{2}\left(1-\frac{1}{\sqrt{1-\kappa^{2}}}\right)}\right)^{2}}\right\rfloor, \quad \kappa=1-1 / n . \quad n<n^{*} \approx 1.46
$$


The numerical solution of $d$ and the approximate solution in (59) are plotted in Figure 6. It can be seen that (59) is an excellent approximation except for $0.5<n<0.6$ where a small discrepancy is observed. For the special case of a neo-Hookean solid with $n=1$,

$$
d=1 \text { and } g(\theta, n=1)=\cos \theta .
$$

For $n>n^{*}, g(\theta, n)$ is obtained by numerically solving a complicated differential equation given in Long et al. [46]. Plots of $g(\theta, n)$ for various $n$ smaller or larger than $n^{*}$ are shown in Figure 7. Note that $g(\theta, n)$ for $n=3$ and $n=6$ has very similar behavior, suggesting the rapid convergence of $g$ for $n$ greater than 6 . It is interesting to note that although the plane stress solution of $y_{2}$ in (58b) is exactly the same as that of the plane strain solution in $(35 \mathrm{~b})$, the $y_{1}$ here has different behavior from that of the plane strain solution in (35a), in contrast to LEFM.

Using (58a,b) and (38b), the crack opening profile is given by:

$$
y_{2}=\left\{\begin{array}{ll} 
\pm a n^{\frac{1-n}{2 n}}\left|\frac{y_{1}}{c g(\pi, n)}\right|^{\frac{2 n-1}{2 n d}} & n<n^{*} \\
\pm\left(a n^{\frac{1-n}{2 n}}\right)^{\frac{6 \mathrm{n}}{4 \mathrm{n}+1}}\left|\frac{y_{1}}{g(\pi, n)}\right|^{\frac{4 n-2}{4 n+1}} & n>n^{*}
\end{array} .\right.
$$

Note that physically we expect $y_{1}<0$ when $\theta=\pi$ (see Figure 1b). This is satisfied since $g(\pi, n)<0$ as shown in Figure 7 . The crack opening profile, specifically the scaling relation between $y_{1}$ and $y_{2}$ is different from that of the plane strain solution in $(40 \mathrm{a}, \mathrm{b})$. The only exception is for $n=1$ where both plane stress and plane strain solutions states that the crack opens to a parabola shape. Similar to plane strain, with sufficient stiffening (i.e., $n>n^{*}$ ), the displacement fields is determined by a single loading parameter $a$, otherwise it depends on both $a$ and $c$.

Geubelle and Knauss [45] also derived the mixed-mode solution for a limited range of $n$. Following Stephenson's approach, they found that the leading order terms of $y_{1}$ and $y_{2}$ are the same as those given in (54). The results can also be expressed in terms of the canonical form $y_{1}^{*}$ and $y_{2}^{*}$ defined in (55). For $n<n^{*}$, the first order terms of $y_{1}^{*}$ and $y_{2}^{*}$ are the same as those in 
(58a,b). Geubelle and Knauss [45] also obtained higher order terms of $y_{1}^{*}$ and $y_{2}^{*}$ which is not detailed here. For $n>n^{*}$ no mixed-mode solution is available to the best of our knowledge.

\subsubsection{Crack tip stress field for GNH solids (Mode I)}

We start with the nominal stress fields $\sigma_{\alpha \beta}$. For all $n>1 / 2$, the dominant nominal stress components are $\sigma_{2 \beta}$ and are the same as those given in $(41 \mathrm{a}, \mathrm{b})$ with $A$ replaced by $A=(\mu / 2) b^{n-1} n^{-n}$. Therefore, the dominant singular nominal stress component has the same form in plane strain and plane stress. Recall that $b$ is a material constant of the GNH model in (13). For $n<n^{*}$, the subdominant components of the nominal stress components $\sigma_{1 \beta}$ follows the asymptotic behaviors listed below:

$$
\sigma_{11}=O\left(r^{d-2+\frac{1}{n}}\right) \text { and } \sigma_{12}=O\left(r^{d-2+\frac{1}{n}}\right) \text {. }
$$

For $n>n^{*}$, the asymptotic behaviors of the subdominant components $\sigma_{1 \beta}$ are

$$
\sigma_{11}=O\left(r^{-1+\frac{5}{4 n}}\right) \text { and } \sigma_{12}=O\left(r^{-1+\frac{5}{4 n}}\right)
$$

The angular variations of $\sigma_{1 \beta}$ depends on the function $g(\theta, n)$ in $(58 \mathrm{a})$ and cannot be written in closed form.

Next we discuss the true stress fields. It was found in Long et al. [46] that the dominate component is $\tau_{22}$ and is given by (for all $n>1 / 2$ )

$$
\tau_{22}=r^{-1} \mu \frac{b^{n-1}}{n^{n}} a^{2 n}\left(1-\frac{1}{2 n}\right)^{2 n} n^{1-n}[\omega(\theta, n)+\kappa \cos \theta]^{-1} .
$$

Note that this is exactly the same as the plane strain result in (46c) if we replace $A$ by $A=(\mu / 2) b^{n-1} n^{-n}$. For $n<n^{*}$, the subdominant true stress components $\tau_{11}$ and $\tau_{12}=\tau_{21}$ are

$$
\tau_{11}=O\left(r^{2 d-3+\frac{1}{n}}\right), \quad \tau_{12}=O\left(r^{d-2+\frac{1}{2 n}}\right)
$$

For $n>n^{*}$, these stresses are:

$$
\tau_{11}=O\left(r^{-1+\frac{3}{2 n}}\right), \quad \tau_{12}=O\left(r^{-1+\frac{3}{4 n}}\right)
$$


It is important to note that the above true stresses are written in terms the undeformed coordinates $r$ and $\theta$.

\subsubsection{Special case: $\mathbf{n}=1$ for neo-Hookean solid}

When $n=1$, the GNH model in (13) reduces to the neo-Hookean model. Unlike the plane strain case, in plane stress the neo-Hookean model is not equivalent to the Mooney-Rivlin model. For this case, $(58 \mathrm{a}, \mathrm{b})$ become

$$
y_{1}=\operatorname{cr} \cos \theta, y_{2}=a \sqrt{r} \sin (\theta / 2) \text {. }
$$

This implies that the crack opens to a parabolic shape given by

$$
y_{2}= \pm a \sqrt{\frac{-y_{1}}{c}}, \quad y_{1} \leq 0 .
$$

This parabolic crack opening shape has been experimentally observed by Livne et al. [25] for fast cracks with propagation velocity about $43 \%$ of the shear wave speed where inertial effects are not significant. The leading order terms of the nominal stresses are

$$
\sigma_{11}=\mu c, \sigma_{12}=o(1), \sigma_{21}=-\frac{\mu a}{2} r^{-1 / 2} \sin (\theta / 2), \sigma_{22}=\frac{\mu a}{2} r^{-1 / 2} \cos (\theta / 2),
$$

which are practically the same as the plane strain results. The true stress components are:

$$
\tau_{11}=\mu c^{2}, \tau_{12}=\tau_{21}=-\frac{\mu}{2} a c r^{-1 / 2} \sin (\theta / 2), \tau_{22}=\frac{\mu}{4} a^{2} r^{-1}
$$

These asymptotic results are similar to those in plane strain deformation except $\tau_{11}$.

\subsubsection{Exponentially hardening solid (Mode I): Crack tip deformation fields}

For the exponentially hardening solid (see (14)), the crack tip deformation fields $y_{1}$ and $y_{2}$ are not expected to follow the asymptotic expansion in (33). Long et al. [46] solved for the leading orders of $y_{1}$ and $y_{2}$ under Mode-I loadings using a method based on the hodograph transform [70]. For $y_{2}$, the leading order behavior can be approximated by

$$
y_{2} \approx r \sqrt{-J_{n} \ln \left(\frac{r}{A_{2}}\right)} f(\theta),
$$

where 


$$
f(\theta) \approx\left\{\begin{array}{cc}
1 & \theta \in[\pi / 2, \pi] \\
\sin \theta & \theta \in(-\pi / 2, \pi / 2) . \\
-1 & \theta \in[-\pi,-\pi / 2]
\end{array}\right.
$$

In (69a), $A_{2}>0$ is an unknown constant with dimension of length which depends on the external loading and specimen geometry, and $J_{n}$ is a dimensionless material constant describing the severity of strain hardening (see (14)). We emphasize that this solution is expected to be valid only for small $r$ so that $\ln \left(r / A_{2}\right)<0$. Equation (69a) indicates that the region surrounding the crack tip can be divided into three sectors (see Figure 8) with distinct behaviors of $y_{2}$. The leading order behavior $y_{1}$ was found to be

$$
y_{1} \approx r\left(-J_{n} \ln \left(\frac{r}{A_{2}}\right)\right)^{-1 / 4} G(\theta),
$$

where $G(\theta)$ is an even function and can only be determined up to an arbitrary constant. Specifically, its value at $\theta=0, G_{0} \equiv G(\theta=0)$, depends on the applied load and specimen geometry. An example plot of $\bar{G}(\theta)=G(\theta) / G_{0}$ and $f(\theta)$ versus $\theta$ was given in Long et al. [46] and is shown here in Figure 9. Also plotted are results of a finite element model (FEM) in Long et al. [46]. Figure 9 shows that the asymptotic solutions of $G(\theta)$ and $f(\theta)$ qualitatively captures the behaviors exhibited in the FEM results, but the quantitative discrepancy is not small. This is expected for two reasons. First, the leading order solutions of $y_{1}$ and $y_{2}$ dominate over their higher order terms only by weak logarithmic singularities. This means that effects of higher order terms can be important unless $r$ is extremely small. Second, (69a,b) and (70) are only approximate solutions in the sense that the detailed deformation fields within the boundary layers between different regions (see Figure 8) are not resolved in these solutions. Overall, (69a,b) and (70) can serve as a first order estimate of the crack tip deformation field.

The crack opening profile is given by setting $\theta= \pm \pi$ and we found, after some calculations, that

$$
\bar{y}_{2} \approx-\bar{y}_{1}\left[-\ln \left(-\bar{y}_{1}\right)\right]^{1 / 4} \sqrt{-\ln \left(-\bar{y}_{1}\left[-\ln \left(-\bar{y}_{1}\right)\right]^{1 / 4}\right)},
$$

where 


$$
\bar{y}_{2} \equiv \frac{y_{2}}{A_{2} \sqrt{J_{n}}} \quad \text { and } \quad \bar{y}_{1}=\frac{J_{n}^{1 / 4} y_{1}}{A_{2}|G(\theta=\pi)|}<0 .
$$

Again $\left|\bar{y}_{1}\right|$ should be sufficiently small so that $\ln \left(-\bar{y}_{1}\right)<0$.

\subsubsection{Exponentially hardening solid (Mode I): Crack tip stress fields}

Based on the asymptotic behaviors of $y_{\alpha}$, Long et al. [46] also derived the leading order terms of the nominal stress fields $\sigma_{\alpha \beta}$ and the true stress fields $\tau_{\alpha \beta}$. Here we highlight the leading order terms of the dominant true stress component $\tau_{22}$ which is particularly relevant for the Mode-I crack deformation. In Regions I and III, i.e. $\theta \in[\pi / 2, \pi]$ or $[-\pi,-\pi / 2]$, the leading order term of $\tau_{22}$ are

$$
\tau_{22}=-\frac{2 \Omega}{J_{n}} \frac{\cos \theta}{r}, \text { and } \Omega=\mu A_{2} \exp \left(-3 / J_{n}\right) \text {. }
$$

In Region II, i.e., $\theta \in(-\pi / 2, \pi / 2)$, the leading order term of $\tau_{22}$ is

$$
\tau_{22}=\frac{\Omega}{-J_{n} r \ln \left(r / A_{2}\right)} .
$$

Recall that $A_{2}$ is the undetermined constant in (69a). Interestingly, $\tau_{22}$ shows slightly different singularities in different regions: $\sim 1 / r$ in Region I and III and $\sim 1 / r \ln \left(r / A_{2}\right)$ in Region II. This behavior was shown to be consistent with finite element results in Long et al. [46]. Interestingly, for the GNH solids, $\tau_{22}$ also exhibits a $1 / r$ singularity at the crack tip which is independent of the strain hardening exponent $n$ (see (63) in Section 3.3.2). Indeed, it was argued in Long et al. [46], based on the path-independence of J-integral, that the true stress component $\tau_{22}$ might

feature a universal $1 / r$ singularity, at least in some angular sector (in terms of $\theta$ ) around the crack tip, for Mode-I cracks in any hyperelastic materials.

\subsection{Anti-plane shear crack in homogeneous materials}

As discussed in Section 2.1, under anti-plane shear the only non-vanishing displacement component is the out of plane displacement $w$, which is assumed to depend only on the in-plane 
coordinates $X_{\alpha}$. For a traction free crack under anti-plane shear loading, Knowles [47] showed that the out of plane shear nominal and true stresses are the same, i.e.,

$$
\sigma_{\alpha 3}=\sigma_{3 \alpha}=\tau_{3 \alpha}=\tau_{\alpha 3}=\left.2 \frac{d W}{d I}\right|_{I=3+\left(\partial w / \partial X_{1}\right)^{2}+\left(\partial w / \partial X_{2}\right)^{2}} \frac{\partial w}{\partial X_{\alpha}} .
$$

All the in-plane stresses must be zero: $\sigma_{\alpha \beta}=\tau_{\alpha \beta}=0$. It should be noted that even though the nominal stress component $\sigma_{33}=0$, the corresponding true stress component is non-zero and is in general unbounded at the crack tip, that is,

$$
\tau_{33}=\left.2 \frac{d W}{d I}\right|_{I=3+\left(\partial w / \partial X_{1}\right)^{2}+\left(\partial w / \partial X_{2}\right)^{2}}\left\lfloor\left(\frac{\partial w}{\partial X_{1}}\right)^{2}+\left(\frac{\partial w}{\partial X_{2}}\right)^{2}\right\rfloor .
$$

Comparing (74) and (75), it is seen that $\tau_{33}$ is more singular than $\tau_{3 \alpha}$. Governing equations and boundary conditions for the crack tip field can be derived based on (5), (10) and (19). Detailed derivation can be found in Knowles [47]. As noted by Knowles, the existence of non-trivial solution for anti-plane shear deformation depends on the choice of strain energy density functions. Fortunately, the class of isotropic strain energy density functions consistent with antishear deformation are those that depends only on $I \equiv I_{1}$ which is the main interest of this review. In this section, we will first summarize the crack tip solutions for GNH solids obtained by Knowles [47] and then those for Gent solid from Long and Hui [48]. In light of (74), we only need to present the true stress components $\tau_{3 \alpha}$ for crack tip stress fields.

\subsubsection{Crack tip fields for GNH solids}

For GNH solids with $n>1 / 2$ (see (13)), Knowles [47] obtained the following first order solution for $w$ :

$$
w=\operatorname{ar}^{1-\frac{1}{2 n}} U(\theta, n),
$$

where $U(\theta, n)$ has been defined in (36a). This solution is exactly the same as that for $y_{2}$ under plane strain or plane stress Mode-I deformation. The leading order terms of $\tau_{3 \alpha}$ are similar to those for $\sigma_{2 \alpha}$ under plane strain or plane stress conditions and are

$$
\tau_{3 \alpha}=\mu b^{n-1} n^{-n+1} a^{2 n-1} r^{-1+\frac{1}{2 n}}\left[\hat{I}_{n}(\theta)\right]^{n-1} \eta_{\alpha}(\theta, n),
$$


where $\hat{I}_{n}(\theta), \eta_{1}(\theta, n)$ and $\eta_{2}(\theta, n)$ are given in $(42 \mathrm{a}, \mathrm{b}, \mathrm{c})$, respectively.

\subsubsection{Crack tip fields for Gent solid}

Unlike the GNH solids, the Gent solid exhibit finite extensibility which means that the stress becomes infinite at finite stretch ratios (see (15) in Section 2.2.4). This feature makes it extremely difficult to analyze the stress and deformation field near a crack tip using numerical methods. Indeed there are very few analytic or numerical works on the effect of finite extensibility on the crack tip stress fields. Long and Hui [48] solved the anti-plane shear crack tip fields for Gent solid using the hodograph transform [69, 70].

We first review the solution of the "Small Scale Yielding" (SSY) problem where the applied loads are sufficiently small so that the nonlinear effects to finite deformation is confined to a region much smaller than typical specimen dimensions, e.g. the crack length or the width of the specimen. In this scenario, material points everywhere except those very close to the crack tip are subjected to infinitesimal deformation and Gent model reduces to Hooke's law where $\sigma_{\alpha}=\mu \gamma_{\alpha}$. Here the term "yielding" does not imply plastic deformation; it is used here since the problem we are considering is very similar to the well-known small scale yielding approximation in elastic-plastic fracture mechanics, where the size of the plastic zone is much smaller than all relevant specimen dimensions. In our case, this condition can be translated into a far field boundary condition that, at large $r$, the deformation and stress fields approach the Mode III LEFM fields with an amplitude given by the stress intensity factor $K_{I I I}$ (see Hui and Ruina [74] for a detailed discussion of SSY). Specifically, the far field boundary condition for the SSS problem is

$$
w(r \rightarrow \infty, \theta)=\frac{K_{I I I} \sqrt{2 r / \pi}}{\mu} \sin (\theta / 2) .
$$

It turned out that the behavior of the near tip stress field changes rapidly across the rays at $\theta= \pm \pi / 2$, similar to what was observed for the plane stress Mode-I crack tip solutions with exponentially hardening solid (Section 3.3.4). It is therefore convenient to divide the area surrounding the crack tip into three regions: Region I, II and III, as shown in Figure 8 . The leading order behaviors of the stresses in these regions are:

Region I: $\pi / 2<\theta \leq \pi$, 


$$
\tau_{31}=-\frac{K_{I I I}^{2}}{\pi \mu \sqrt{J_{n}}} \frac{\cos ^{2} \theta}{r}, \tau_{32}=-\frac{K_{I I I}^{2}}{2 \pi \mu \sqrt{J_{n}}} \frac{\sin 2 \theta}{r} .
$$

Region II: $-\pi / 2<\theta<\pi / 2$

$$
\tau_{31} / \tau_{32} \rightarrow 0 \quad \tau_{32}=\frac{K_{I I I}}{2 \sqrt{\pi r}}
$$

Region III: $-\pi \leq \theta<-\pi / 2$

$$
\tau_{31}=\frac{K_{I I I}^{2}}{\pi \mu \sqrt{J_{n}}} \frac{\cos ^{2} \theta}{r}, \quad \tau_{32}=\frac{K_{I I I}^{2}}{2 \pi \mu \sqrt{J_{n}}} \frac{\sin 2 \theta}{r} .
$$

The asymptotic solution in $(79 \mathrm{a}, \mathrm{b}, \mathrm{c})$ appears to be a discontinuous function of $\theta$. However, this discontinuity is due to an approximation to describe the rapidly changing stress fields across $\theta= \pm \pi / 2$. A discussion of this approximation can be found in Long and Hui [48]. The above solution has several interesting features:

i). Due to the SSY condition, the amplitude of the near tip fields (despite their complexity) is completely determined by the applied stress intensity factor $K_{I I I}$.

ii). The near tip stress field rapidly changes behavior across the rays $\theta= \pm \pi / 2$. As a result, the stress fields are non-separable in the sense that there is no single separable function of $r$ and $\theta$ that works for the entire range of $|\theta| \leq \pi$.

iii). In Region II, the stress state is that of pure shear. The dominate stress component in this region has a classical inverse square root singularity and is independent of the polar angle $\theta$. Surprisingly, the stresses are much more singular behind the crack tip, i.e., in Regions I and III, and scale with1/ $r$.

iv). The dominant singularity of the stress field is much more severe than the prediction of LEFM. In particular, the angular variations of the stress field are completely different.

For more general loading conditions, for example, when the applied stresses are sufficiently large so that the SSY condition is violated, the leading order terms of the stresses were found to be [48]:

Region I: $\pi / 2<\theta \leq \pi$,

$$
\tau_{31}=\frac{\mu L_{0} \sqrt{J_{n}}}{r}|q(\theta-\pi / 2)| \cos \theta, \quad \tau_{32}=\frac{\mu L_{0} \sqrt{J_{n}}}{r}|q(\theta-\pi / 2)| \sin \theta .
$$

Region II: $-\pi / 2<\theta<\pi / 2$ 


$$
\tau_{31}<<\tau_{32}, \quad \tau_{32}=\frac{\mu \sqrt{L_{0} J_{n}}}{2 \sqrt{r}} \sqrt{\left|q^{\prime}(0)\right|} .
$$

Region III: $-\pi \leq \theta<\pi / 2$,

$$
\tau_{31}=-\frac{\mu L_{0} \sqrt{J_{n}}}{r}|q(\theta+\pi / 2)| \cos \theta, \quad \tau_{32}=-\frac{\mu L_{0} \sqrt{J_{n}}}{r}|q(\theta+\pi / 2)| \sin \theta .
$$

In (80a-c), $L_{0}$ is a characteristic length for the crack or the specimen geometry, and $q$ is an unknown function that can only be specified by far field conditions such as specimen geometry and applied loading. This result is interesting since one would expect that the stress fields are similar to those given by (79a-c); only the amplitude needs to be adjusted. Indeed, one of the central premises of the fracture mechanics approach is that the crack tip field should be characterized a small number of loading parameters that completely determines its behavior; other than these parameters, the angular variation and the singular behavior of the crack tip field should not depend on the external loading and specimen geometry. Therefore the Gent solid provides a counter-example to this premise.

\subsection{Interface crack}

Knowles \& Sternberg [49] performed the first study of finite strain crack tip fields for interface cracks between two dissimilar materials. They considered plane stress cracks between two incompressible neo-Hookean materials sheets. Their study was extended by Gebuelle \& Knauss [50, 51] to generalized neo-Hookean materials. In addition, Geubelle \& Knauss [50] considered a special case where the interface crack is between a GNH material and a rigid substrate. We will focus on this special case since it is of particular relevance to the understanding of adhesion between soft adhesives and stiff substrates. It should be noted that there are also a number of finite strain analyses for interface cracks using compressible material models $[52,53,54,55]$, which is not the focus of this review.

The geometry of the interface crack is illustrated in Figure 10a. Geubelle and Knauss [50] assumed zero lateral slip on the interface and found the following asymptotic solutions for the deformation coordinates $y_{1}$ and $y_{2}$ :

$$
\begin{aligned}
& y_{1}=a_{1} r^{1-\frac{1}{2 n}} U(\theta, n)+r v_{1}(\theta, n)+o(r), \\
& y_{2}=a_{2} r^{1-\frac{1}{2 n}} U(\theta, n)+r v_{2}(\theta, n)+o(r),
\end{aligned}
$$


where $a_{1}$ and $a_{2}$ are arbitrary constants, $U(\theta, n)$ is given in (36a), and

$$
v_{1}(\theta, n)=\frac{a_{2}^{2} \bar{v}_{1}+a_{1}^{2} \bar{v}_{2}}{a_{1}^{2}+a_{2}^{2}}, \quad v_{2}(\theta, n)=\frac{a_{1} a_{2}\left(\bar{v}_{1}-\bar{v}_{2}\right)}{a_{1}^{2}+a_{2}^{2}},
$$

with

$$
\bar{v}_{2}(\theta, n)=\frac{\omega(\theta, n)+\kappa \cos \theta}{(1+\kappa) \cos \left(\sqrt{\frac{4 n}{2 n-1} \frac{\pi}{2}}\right)} \cos \left(\sqrt{\frac{4 n}{2 n-1}} \xi_{0}\right) .
$$

Note that $\kappa, \omega(\theta, n)$ and $\cos \xi_{0}$ have been defined in (36b), (36c) and (37c), respectively. There is no closed form solution for $\bar{v}_{1}(\theta, n)$ except for $n=1$, where $\bar{v}_{1}(\theta, n=1)=\cos \theta$, and numerical solutions were presented in Geubelle and Knauss [50]. The solution of $\bar{v}_{2}(\theta, n)$ breaks down when

$$
\sqrt{\frac{4 n}{2 n-1}}=3,5,7 \ldots \quad \text { or } n=\frac{j^{2}}{2\left(j^{2}-2\right)}(j=3,5,7 \ldots) \text {. }
$$

This corresponds to a discrete set of $n=0.6429,0.5436,0.5213$ and so on. For these cases Geubelle and Knauss [50] also presented a different form of asymptotic solutions. It should also be noted that Geubelle and Knauss obtained the third-order terms in (81a,b), but these terms are only valid for a small range of $n(n<\sim 0.75)$. Materials in this range exhibit weak strain hardening that is unlikely to be encountered near the crack tip and thus are not included here.

Next we consider the special case of neo-Hookean material where $n=1$. For this case, $\bar{v}_{1}=\bar{v}_{2}=\cos \theta$ and the deformed coordinates are

$$
\begin{aligned}
& y_{1}=a_{1} r^{\frac{1}{2}} \sin \left(\frac{\theta}{2}\right)+r \cos \theta, \\
& y_{2}=a_{2} r^{\frac{1}{2}} \sin \left(\frac{\theta}{2}\right) .
\end{aligned}
$$

This is exactly the same as that obtained by Lengyel et al. [75] for a plane strain interface crack between a compressible Blatz-Ko material [96] and a rigid substrate. As pointed out by Lengyel et al. [75], the result of $(84 a, b)$ leads to interesting implications for the crack opening profile which can be written as 


$$
y_{1}=\frac{a_{1}}{a_{2}} y_{2}-\left(\frac{y_{2}}{a_{2}}\right)^{2} .
$$

If we neglected the second order term in (84a), then the crack faces deform into the straight line given by $y_{1}=a_{1} y_{2} / a_{2}$. Figure $10 \mathrm{~b}$ shows the normalized crack opening profiles for different values of $a_{1} / a_{2}$. By including the second term on the right hand side of (84a), the crack opens to a parabolic profile. As $a_{1} / a_{2}$ increases from -0.5 to 2 , the crack surface flips forward with a wedge angle $\zeta<\pi / 2$ (see Figure 10b). From (85), the wedge angle $\zeta$ is related to $a_{1}$ and $a_{2}$ by

$$
\tan (\zeta)=\frac{a_{2}}{a_{1}}, \quad 0 \leq \zeta \leq \pi
$$

The flipping forward behavior of the crack faces has also been observed in the finite element analysis of Kristnan et al. [63] and more importantly, in the experiments of Nase et al. [76] where a soft adhesive was peeled off from a stiff substrate. In addition, using (84a,b) and (9), the true stress field near the crack tip is

$$
\tau_{11}=\mu a_{1}^{2} r^{-1} / 4, \tau_{12}=\tau_{21}=\mu a_{1} a_{2} r^{-1} / 4, \tau_{22}=\mu a_{2}^{2} r^{-1} / 4
$$

Note all three stress components have the same $r^{-1}$ singularity. The relative ratios of these stress components are related to the wedge angle $\zeta$ by

$$
\frac{\tau_{22}}{\tau_{11}}=(\tan \zeta)^{2}, \quad \frac{\tau_{12}}{\tau_{11}}=\tan \zeta
$$

All three in-plane stress components have the same amplitude when $a_{1}=a_{2}$ or $\zeta=\pi / 4$. For wedge angle less than $\pi / 4$, the component $\tau_{11}$ has the largest amplitude, while for wedge angle greater than $\pi / 4$, the component $\tau_{22}$ is the largest.

The above results are based on the assumption the interface is perfectly bonded so there can be no lateral slip. The crack tip stress field may become dramatically different had there be any interface slippage which can have a significant effect on the fracture energy between a soft adhesive and a rigid substrate. For example, Newby et al. [77] found that the apparent fracture energy of a soft adhesive on a fluorocarbon functionalized (FC) silicon wafer was significantly greater than that on a hydrocarbon (HC) functionalized silicon wafer, even though the FC functionalized surface has the lower surface free energy. They proposed that an interface that allows easier slippage (HC) dissipates less energy than one that is more slip resistant (FC). 
To gain insight into this interesting phenomenon, we consider the limiting case where the interface has no resistance to shear and the solid is allowed to slip freely. This is equivalent to the Mode-I condition presented in Section 3.3.3, where the normal stress component $\tau_{22}\left(\sim r^{-1}\right)$ dominates over the other two $\tau_{12}\left(\sim r^{-1 / 2}\right)$ and $\tau_{11}$ (bounded). A similar comparison and further discussions for plane strain crack with compressible Blatz-Ko materials were made in Lengyel et al. [75].

\section{True stress in deformed coordinates}

In Section 3.2 and 3.3, the asymptotic solutions of true stress $\tau_{\alpha \beta}$ are expressed in terms of the undeformed polar coordinates $(r, \theta)$. In practice, the distribution of true stress in the deformed configuration is more relevant to the interpretation of experimentally observed crack deformation and growth behaviors. In light of this consideration, we set up a deformed polar coordinate system $(\rho, \phi)$ at the deformed crack tip (see Figure 1B), and discuss the crack tip true stress field in terms of these coordinates. A material point originally at $(r, \theta)$ is displaced to $(\rho$, $\phi)$, which can be calculated using

$$
y_{1}=\rho \cos \phi, y_{2}=\rho \cos \phi,
$$

and the asymptotic solutions of $y_{\alpha}(r, \theta)$. We will focus on plane strain or plane stress Mode I cracks with GNH solids.

\subsection{Plane strain Mode I}

We start with the plane strain Mode I crack tip fields presented in Section 3.2.2 and 3.2.3. For $1 / 2<n<3 / 2$, a straight forward calculation of the Jacobian of the mapping from the undeformed coordinates $(r, \theta)$ to the deformed coordinates $\left(y_{1}, y_{2}\right)$ using the leading order terms of $y_{\alpha}$ shows that it is identically zero. Therefore, the mapping is not one to one unless higher order terms are included in the local asymptotic expansion. For example, if $1 / 2<n<3 / 2$, (35a) and (38a) imply that $y_{1}=0$ for all $r$ when $\theta=0$ which is obviously unphysical. For the special case of $n=1$ (neo-Hookean solid), Stephenson [39] derived the asymptotic solutions of $y_{\alpha}$ up to the third order terms. Here we use the first two terms in the asymptotic expansion and they are 


$$
\begin{aligned}
& y_{1}=-b_{0} r \sin ^{2} \frac{\theta}{2}+\frac{1}{a} r^{3 / 2}\left\lfloor 4 \sin ^{2} \frac{\theta}{2} \cos \frac{\theta}{2}+\frac{4}{3} \cos ^{3} \frac{\theta}{2}\right\rfloor+O\left(r^{2}\right), \\
& y_{2}=a \sqrt{r} \sin \frac{\theta}{2}+\frac{1}{a} r^{3 / 2}\left\lfloor c_{3} \sin \frac{3 \theta}{2}-\frac{1}{2} b_{0}^{2} \sin \frac{\theta}{2}\right\rfloor+O\left(r^{2}\right) .
\end{aligned}
$$

Note that $a>0$ and $b_{0}>0$ and $c_{3}$ is a constant coefficient which cannot be determined by asymptotic analysis. With these second order terms included, it can be readily checked that the Jacobian of the mapping from $(r, \theta)$ to $\left(y_{1}, y_{2}\right)$ is non-zero for $r>0$. A plot to illustrate the mapping is shown in Figure 11A and 11B.

To obtain the true stress distribution in terms of $\rho$ and $\phi$ for a neo-Hookean solid, we need to first use $(89 \mathrm{a}, \mathrm{b})$ to solve $r$ and $\theta$ explicitly and then apply the asymptotic solutions in (50). Distribution of the true stress components in the undeformed and deformed configurations can be visualized in the contours plots of Figure 12. However, even for the neo-Hookean model, there is no close-form equation expressing the singular components of true stress in terms of the deformed coordinates $\rho$ and $\phi$. To gain some insights on the stress distribution, let us consider the material points directly ahead of the crack tip $(\theta=0)$ or along the crack faces $(\theta= \pm \pi)$. For the line directly ahead the crack tip, we have

$$
y_{1}=\frac{4}{3 a} r^{3 / 2}, y_{2}=0 \Rightarrow \rho=\frac{4}{3 a} r^{3 / 2}, \phi=0 .
$$

Using (90a), the opening true stress directly ahead of the crack tip is

$$
\tau_{22}(r, \theta=0)=\frac{\mu a^{2}}{4} r^{-1} \Leftrightarrow \tau_{22}(\rho, \phi=0)=\frac{\mu a^{4 / 3}}{4}\left(\frac{3}{4}\right)^{-2 / 3} \rho^{-2 / 3} .
$$

Next, consider the undeformed crack faces at $\theta= \pm \pi$, it is deformed to

$$
y_{1}=-b_{0} r, y_{2}= \pm a \sqrt{r} \quad \Rightarrow \rho=a \sqrt{r}, \phi= \pm \pi / 2 \text {. }
$$

Note that when deriving $\rho$ and $\phi$ above, we have neglected $y_{1}$ as compared to $y_{2}$ since $r$ approaches 0 . Therefore, we have

$$
\tau_{22}(r, \theta= \pm \pi)=\frac{\mu a^{2}}{4} r^{-1} \Leftrightarrow \tau_{22}(\rho, \phi= \pm \pi / 2)=\frac{\mu a^{4}}{4} \rho^{-2} .
$$

According to (50), when expressed in terms of the undeformed coordinates, $\tau_{22}$ is independent of $\theta$ and is inversely proportional to $r$. This is clearly not the case when $\tau_{22}$ is expressed as a function of deformed coordinates, as can be seen in Figure 12F. In terms of the deformed 
coordinates $\rho$ and $\phi, \tau_{22}$ is non-separable and the stress singularity exponent changes as one approaches the crack tip, depending on the direction of approach. It is particular interesting to note that, directly ahead of the crack tip, $\tau_{22}$ has a weaker singularity in the deformed coordinates $\left(\tau_{22} \sim \rho^{-2 / 3}\right.$ versus $\tau_{22} \sim r^{-1}$ ). However, if one approaches the crack tip along the deformed crack faces, the singularity is actually more severe $\left(\tau_{22} \sim \rho^{-2}\right)$.

Although the use of undeformed coordinates $(r, \theta)$ as independent variables simplifies the representation of the crack tip stress fields, it can cause confusion in interpretation of experimental data. For example, Trabelsi et al [78] performed fracture experiments in natural rubber. They compared their experimentally measured singularity exponent ( $\tau_{22}$, directly ahead of the crack tip) of -0.7 with the singularity exponent of -1 given by the solution of a neoHookean solid in (50). As noted above, this singularity exponent of -1 corresponds to the undeformed coordinate $r$. Since the measurements are conducted in the deformed configuration, it is more appropriate to use deformed coordinate $\rho$ as the independent variable. According to our discussion above (see (90b)), the dominate stress $\tau_{22} \sim \rho^{-2 / 3}$ and has an exponent of $-2 / 3 \sim$ -0.67 , which is in reasonable agreement with their measurements $(-0.7)$.

For $n>3 / 2$, the first order asymptotic solutions in (35a,b) do give a one-to-one mapping from the undeformed coordinates to the deformed coordinates. However, it is still not possible to explicitly express $r$ and $\theta$ in terms of $\rho$ and $\phi$. Therefore, we consider the special case of $\theta=0$ which corresponds to $\phi=0$. From (35b), we have

$$
y_{1}=\rho=-\frac{1}{a} r^{1+\frac{1}{2 n}} H(\theta=0, n),
$$

where $H(\theta=0, n)<0$ and is given in (39a). Using (92a) and (46c), we found that directly ahead of the crack tip:

$$
\tau_{22}=2 A m^{\frac{2 n(2 n-1)}{2 n+1}} n^{1-n}\left[n^{3 / 2} /(2 n+1)\right]^{2 n /(2 n+1)}\left(\frac{a^{2 n}}{\rho}\right)^{\frac{2 n}{2 n+1}}
$$

This result shows that $\tau_{22}$ has the weaker stress singularity exponent of $-2 n /(2 n+1)$ in terms of the deformed coordinate $\rho$ as compared to $\tau_{22} \sim r^{-1}$. 


\subsection{Plane stress Mode I}

For plane stress Mode I cracks, it is also in general not possible to find closed-form expressions for the true stress as a function of the deformed coordinates $\rho$ and $\phi$, since $g(\theta, n)$ in (58a) can only be solved numerically. The only exception is the special case of neo-Hookean solid with $n=1$. This was discussed in Krishnan et al. [62] and the results are summarized here. The following relations were found based on the first order solutions in (65):

$$
r=\frac{2 \rho^{2} \sin ^{2} \phi}{a^{2}}+\frac{\rho \cos \phi}{c}, \quad \cos \theta=\frac{a^{2} \cos \phi}{2 c \rho \sin ^{2} \phi+a^{2} \cos \phi} .
$$

Recall that $a$ and $c$ are unknown constants in (65). Unlike the plane strain case, (93) implies that there is a one to one correspondence between $\theta$ and $\phi$. In particular, $\theta= \pm \pi$ corresponds to $\phi= \pm \pi / 2$. An illustration of the mapping is shown in Figure 13. Substituting (93) into (68) results in

$$
\begin{gathered}
\tau_{22}=\frac{\mu a^{4} c}{4 \rho}\left\lfloor\frac{1}{2 c \rho \sin ^{2} \phi+a^{2} \cos \phi}\right\rfloor, \quad 0 \leq|\phi| \leq \pi / 2, \\
\tau_{12}=\tau_{21}=-\mu \frac{a^{2} c^{3 / 2}}{2 \sqrt{2}} \frac{\operatorname{sign}(\phi)}{\sqrt{\rho}}\left(\frac{1}{2 c \rho \sin ^{2} \phi+a^{2} \cos \phi}\right)^{1 / 2}\left(1-\frac{a^{2} \cos \phi}{2 c \rho \sin ^{2} \phi+a^{2} \cos \phi}\right)^{1 / 2}
\end{gathered}
$$

where $\operatorname{sign}(\phi)$ is 1 if $\phi>0$ and -1 if $\phi<0$. The component $\tau_{11}$ is not listed here since its first order term is a constant. Contour plots of $\tau_{12}$ and $\tau_{22}$ in undeformed and deformed configurations are shown in Figure 14. The results above suggest that there is a boundary layer near the deformed crack surfaces. Specifically, if we set $\phi=\pi / 2$, then $\tau_{12}$ and $\tau_{22}$ on the deformed upper crack face are:

$$
\tau_{12}(\rho, \phi=\pi / 2)=-\mu \frac{a^{2} c}{4} \frac{1}{\rho}, \quad \tau_{22}(\rho, \phi=\pi / 2)=\mu \frac{a^{4}}{8 \rho^{2}} \text { at } \phi=\pi / 2 .
$$

On the other hand, in regions that are away from the crack surfaces, we can take advantage of the fact that $\rho$ is asymptotically small and obtain the following results

$$
\tau_{12}=-\mu \frac{c^{2}}{2} \tan \phi, \quad \tau_{22}=\frac{\mu a^{2} c}{4 \rho} \frac{1}{\cos \phi}, \text { for }|\phi|<\pi / 2 .
$$

Note that the shear stress is bounded and is independent of the radial distance $\rho$ in this sector $-\mathrm{a}$ surprising result since the shear stress is unbounded when expressed in $(r, \theta)$ (see (68)). It is also interesting to compare with the plane strain result for the neo-Hookean solid. The singularity 
exponents in $\tau_{22}(\rho, \phi)$ for plane strain and stress at $\phi= \pm \pi / 2$ are both -2 . However, the singularity exponent of $\tau_{22}(\rho, \phi)$ at $\phi=0$ for plane strain is $-2 / 3$ whereas it is -1 for plane stress.

\section{Energetics: J-integral and energy release rate}

Eshelby [79] has first shown that the J-integral is a surface independent integral which can be used to determine the energetic forces on defects. Rice [80] developed the same integral for infinitesimal deformation and successfully applied it to the analysis of notches and cracks. For elastic solid with a structureless crack tip, the J-integral, when specialized to plane strain or plane stress, is found to be path independent and can be identify as the energy release rate. By "structureless" we meant that the crack surfaces remain traction free all the way to the crack tip and we exclude other irreversible effects due to microstructural damage which in reality must take place since the stresses cannot be infinite. In this damage region, the material is not elastic and hence the J-integral is not necessarily path independent. In addition, if the material directly ahead of the crack tip decoheres, then the path of the J-integral must lie outside the region of decohesion. In the context of finite strain elastostatics, the J-integral is defined as follows [31]:

$$
J=\int_{C}\left(W N_{1}-\frac{\partial u_{\alpha}}{\partial x_{1}} \sigma_{\alpha \beta} N_{\beta}\right) d S,
$$

where $C$ is a smooth contour in the reference configuration with arc length $S$, and $N_{\beta}$ are components of the unit normal vector of $C$ (see Figure 15). For anti-plane shear, the J-integral is

$$
J=\int_{C}\left(W N_{1}-\frac{\partial u_{3}}{\partial x_{1}} \sigma_{3 \beta} N_{\beta}\right) d S
$$

\subsection{Evaluation of J-integral}

To evaluate the J-integral, we pick $C$ to be a circular path of radius $\varepsilon$ so that $d S=\varepsilon d \theta$. In addition, $\varepsilon$ is sufficiently small so that the first order asymptotic solutions of deformation and stress fields dominate on this path. For the plane strain Mode-I crack reviewed in Section 3.2, we found

$$
J=\pi A\left(\frac{2 n-1}{2 n}\right)^{2 n-1} n^{2-n} a^{2 n} \quad \text { (Plane strain Mode I ) }
$$


Recall that $A$ and $n$ are material parameters defined in (34) [39], and $a$ is the amplitude of the leading order term of $y_{2}$ in $(35 \mathrm{~b})$. For plane stress Mode-I crack in GNH solids (see Section 3.3), the $J$-integral can be derived similarly was explicitly given in Long et al. [46]. In our notation, the result is

$$
J=\frac{\mu \pi}{2}\left(\frac{b}{n}\right)^{n-1}\left(\frac{2 n-1}{2 n}\right)^{2 n-1} n^{1-n} a^{2 n} \quad \text { (Plane stress Mode I) }
$$

where $b$ and $n$ are material constants of GNH solids (see (13)) and $a$ is the amplitude of the leading order term of $y_{2}$ in $(58 \mathrm{~b})$. Note that the plane stress result is the same as the plane strain result if one replaces $A$ by $A=(\mu / 2) b^{n-1} n^{-n}$. For the special case of $n=1$, that is, for a neoHookean solid, the plane strain and plane stress Mode-I J-integral has the same form:

$$
J=\frac{\mu \pi a^{2}}{4} .
$$

In both the plane stress and plane strain Mode-I cases, the amplitude $a$ not only controls the amplitude of the dominant singular stress component, but also determines the J-integral. For the exponentially hardening material, Long et al. [46] found

$$
J=\frac{\mu \pi \mathrm{e}^{-3 / J_{n}}}{J_{n}} A_{2}, \quad \text { (plane stress Mode I), }
$$

where $J_{n}$ is a material parameter (see (14)) and $A_{2}$ is the undetermined coefficient (with dimension of length) in (69a).

\subsection{Interpretation of J-integral in Experiments}

As illustrated above, the amplitude of the dominant asymptotic solution (e.g. $a$ ) can be related to the J-integral, which may be determined by experiments. For example, a widely used geometry is the pure shear crack specimen which consists of a thin elastic sheet in the form of an infinite long rectangular strip. The strip has a semi-infinite crack in the mid-plane (see Figure 16). The upper and lower boundaries of the strip are clamped by rigid grips. Mode I loading can be applied by moving the grips vertically apart by $\pm \Delta$. Since the sheet is thin, i.e., its thickness is small in comparison with all relevant specimen dimensions, the pure shear specimen can be regarded as being loaded in plane stress. The J-integral for this case can be evaluated using the following formula [1] 


$$
J=2 W\left(I_{1}, I_{2}\right) h_{0}=2 \Psi\left(\lambda_{A}\right) h_{0} \quad \text { where } \quad I_{1}=I_{2}=\lambda_{A}^{2}+\lambda_{A}^{-2}+1, \lambda_{A}=1+\frac{\Delta}{h_{0}} .
$$

where $2 h_{0}$ is the height of the undeformed strip and $\lambda_{A}$ is the applied stretch ratio on the strip sample. The function $\Psi\left(\lambda_{A}\right)$ can be determined by measuring the nominal stress $\sigma(\lambda)$ of a thin sheet subjected to uniaxial stretch $\lambda$ and is the area under the $\sigma(\lambda)$ versus $\lambda$ curve, that is:

$$
\Psi\left(\lambda_{A}\right)=\int_{1}^{\lambda_{A}} \sigma(\lambda) d \lambda
$$

This result can be easily obtained by noting that the fields are translationally invariant: one can grow the crack a unit distance by removing a unit width of the strip at $X_{1}=\infty$ and depositing the removed material at negative infinity $X_{1}=-\infty$. Since the material is elastic, material at negative infinity carries no energy, so the energy release per unit crack extension during this process is the strain energy per unit length stored at $X_{1}=\infty$, which is $2 \Psi\left(\lambda_{A}\right) h_{0}$.

For a neo-Hookean solid, $\Psi\left(\lambda_{A}\right)=\mu\left(\lambda_{A}-\lambda_{A}^{-1}\right)^{2} / 2$. Using (98) and (100) we can determine amplitude $a$ of the asymptotic solution in (65) for the strip geometry shown in Figure 16 , that is,

$$
a=2 \sqrt{\frac{h_{0}}{\pi}}\left(\lambda_{A}-\lambda_{A}^{-1}\right)
$$

The constant $c$ in (65) cannot be determined using the $J$ integral. Krishnan et al. [62] numerically determined $c$ and showed that it decreases monotonically from 1.55 at $\lambda_{A}=1.02$ to 1.15 at $\lambda_{A}=2$.

The $J$ integral can be interpreted as the energy flux to a structureless crack tip growing in a purely elastic solid under steady state conditions. In reality, all materials are dissipative to a certain extent. However, as long as deviation from elasticity is confined to a region near the crack tip that is very small in comparison with typical dimensions of the specimen, it is still possible to regard the $\mathrm{J}$-integral as energy release rate. But this is not the case for some recently developed tough soft materials such as the double network gels of Gong et al. [7] and Sun et al [8]. In these gels, the breakage of sacrificial bonds is not confined to the crack tip and occupies a significant volume of the specimen. Likewise, for materials that exhibits significant time dependent behavior, such as viscoelastic flow, the J-integral is neither path independent nor can it be interpreted as the energy release rate to the crack tip. In other words, for dissipative 
materials, there is no theoretical basis for correlating crack growth rate with energy release rate, even if it can be determined empirically.

Nevertheless, there are certain situations where the use of J-integral may be justified. For example, assume the material being studied is not rate dependent, one may use the condition $J=J_{c}$ as a criterion for the onset of crack growth [8]. For the case of a pure shear crack specimen, $J$ can be computed using (100) and (101) and $J_{c}$ is the critical value of $J$ at the onset of crack growth. The rationale lies in the argument that as long as the crack remains stationary, material points in the specimen continues to load, so the material is indistinguishable from a nonlinear elastic solid even if damage occurs. It is important to note that in this case the Jintegral cannot be interpreted as the energy release rate which is only meaningful for continuous crack propagation in an elastic solid. Rather it should be considered as the parameter that controls the dominant singular stress field near the crack tip, as shown above. In other words, the J-integral controls the local deformation near the crack tip, and hence the failure process, assuming that the region of dominance of the asymptotic stress field is large enough to include the fracture process zone. As a result, two different specimens with cracks of different sizes and with different Mode I loadings, will have identical local deformation fields in a common region surrounding the crack tip; as a result, it is expected that onset of crack growth of these specimens will occurs at the same critical $J=J_{c}$.

\section{Summary and Discussion}

In this review we have summarized the finite strain crack tip solutions for various incompressible material models with different geometrical constraints: plane strain, plane stress, anti-plane shear and interface cracks. The connection of J-integral to the crack tip fields and its application in fracture experiments of soft materials was also discussed. An important aspect of the finite strain crack tip fields that was not emphasized in previous literature is the true stress distribution in the deformed configuration, which can be crucial for understanding experimentally observed crack deformation and growth behaviors.

The finite strain crack tip solutions can lead to implications on the detailed failure mechanisms in the crack tip region. Consider a Mode-I plane strain crack. It was shown in Section 3.2.4 (see (50)) that for neo-Hookean solid the true stress component $\tau_{22}$ has the most severe singularity, which means that the crack tip region is effectively under a uni-axial tensile 
stress state. This is completely different from the LEFM solutions which predict a tri-axial tensile stress state directly ahead of the crack tip (see (21c)). As the strain stiffening effect becomes stronger, i.e., with increasing $n$ in the GNH model, the singularity exponents of all the true stress components become closer and the crack tip stress fields approaches a tri-axial stress state. This feature has not been emphasized in previous study [39] even though it is exhibited by the crack tip solution. This transition in stress state may imply distinct material failure mechanisms in the crack tip region. For example, it is known that cavitation typically occurs under hydrostatic tension and is much less likely under uni-axial tension [17]. Therefore, it is speculated that for neo-Hookean solid, cavitation is not expected to occur in the crack tip region. But for materials with strong strain stiffening behavior, cavitation may occur in the crack tip region.

The distribution of true stress in deformed configuration, as discussed in Section 4, also has interesting physical implications. Take neo-Hookean solid for example. The initially sharp crack becomes blunted after deformation (see Figures 11 and 12). The crack surface originally at $\theta=\pi$ is mapped to a vertical surface with $\phi=\pi / 2$ in the crack. On this vertical surface, the dominant stress component $\tau_{22}$ is more singular than the $\tau_{22}$ directly ahead of the crack tip ( $\theta$ $=0)$. As a result, new secondary cracks may initiate on the blunted crack surface and the primary crack may deviate from its direction, leading to tortuous crack propagation path and rough crack surface. This point was first recognized by Krishnan et al. [62], and has been adopted to explain the experimentally observed crack tip splitting phenomenon in elastic acrylic triblock copolymer [64] and crack branching instability in gelatin gels [81] under quasi-static loadings.

Although it is widely accepted that the LEFM solutions do not apply when the crack is highly deformed, there are only a few direct experimental observations demonstrating the breakdown of LEFM. The pioneering work along this direction is by Livne et al. [24, 25] who measured the deformation field near the tip of steadily propagating dynamic crack in a thin sheet of polyacrylamide gel whose elastic response can be well described by the neo-Hookean model up to nominal strains of about $100 \%$. Note that the experiments of Liven et al. [24, 25] were for a wide range of crack velocity, that is, from $20 \%$ to $80 \%$ of the shear wave speed, which covers the transition from the quasi-static limit (slow crack propagation) to the elastodynamic limit (fast crack propagation). It was found that the LEFM could not fully capture the crack tip deformation field and broke down in the vicinity of the crack tip where large deformation prevails. In that 
nonlinear region solutions based on finite elastodynamics were found to agree well with experimental data. On a related note, the breakdown of LEFM has also been explicitly addressed by the weakly nonlinear solution by Bouchbinder et al. [26, 27, 28] which possesses an intermediate region of validity between the LEFM field and the fully nonlinear field. In particular, Livne et al. [25] stressed that for plane stress dynamic cracks with a finite propagation speed, LEFM predicts that the stress component $\tau_{11}$ is always larger than $\tau_{22}$ directly ahead of the crack tip which was deemed unphysical. The opposite is predicted by large deformation solutions. For more details, see equation (15) in the supplementary material of Liven et al. [25] and associated discussions there.

Recently Lefranc and Bouchaud [29] studied the quasi-static deformation of a steadily propagating crack in thin sheets of agar gels. They found that the opening displacement of the crack undergoing steady-state propagation was sensitive to the loading rate. Higher loading rates and hence faster crack propagation result in larger opening displacements. This indicates that the fracture energy is rate-dependent which was attributed to the viscous dissipation processes in the vicinity of the crack tip. For slow cracks, the opening displacement can be well fitted by LEFM solutions. However, for rapid cracks with much larger crack opening, there was an obvious discrepancy between LEFM solutions and experimental data close to the crack tip. The experimental data showed a sharper and wedge-like crack opening shape. The authors mentioned that such wedge-like crack shape observed under quasi-static loading is similar to that computed for the exponentially hardening material in Long et al. [46], which is reviewed in Section 3.3.4 and 3.3.5. However, this potential connection remains to be confirmed by more in-depth and quantitative studies.

There are still many open questions in the area of finite strain crack tip analysis. A few examples are listed below.

- Crack tip fields for soft solids with material heterogeneity which can either be intentionally introduced (e.g. gradients in material property or stiff inclusions), or due to progressive damage near the crack tip. The latter is especially relevant to the recently developed tough hydrogels or elastomers that incorporated sacrificial networks or bonds to enhance energy dissipation during fracture $[7,8,9,10,12]$.

- Near-tip deformation and stress fields in soft materials for three-dimensional (3D) cracks. So far all of the available crack tip solutions are for two-dimensional cracks. It is 
interesting to study how three-dimension effects affect the crack tip fields, which can provide important insights towards understanding the various crack front instabilities observed in experiments $[82,83]$. In addition, the near-tip fields for cracks with a curved front may help better understand the undulatory instability observed for an interface crack between a confined elastomer film and thin elastic plate [84, 85].

- Crack tip fields for anisotropic soft materials. This class of materials includes fiberreinforced elastomers [86] and a wide range of biological tissues such as articular cartilage, tendons and arterial walls [87].

- Effects of time-dependent phenomena on crack tip fields. Most soft polymers exhibit viscoelastic behavior, which was shown to have a significant impact on the fracture behavior [6]. In addition, gels also exhibit poroelastic behavior which means the mechanical deformation can be coupled to diffusion of fluids in polymer matrix. Further studies are needed for the effect of poroelasticiy on crack tip fields to understand fracture of gels [21, 22] and the effects of solvents [88], especially under large deformation.

- Although this review focuses on quasi-static deformation of cracks in soft materials, we note that there have been considerable experimental and theoretical studies on dynamic crack propagation in soft rubbers and gels $[89,90,91,92,93]$. However, analyses for the crack tip field based on finite strain elastodynamics are still limited. The existing solutions are only on plane stress cracks with incompressible neo-Hookean [25], or compressible neo-Hookean and Mooney-Rivlin material models [94, 95]. Efforts are needed to extend the elastodynamic crack tip solutions to a wider class of material models (e.g. models with strain hardening) and geometries (e.g. plane strain or 3D cracks).

These analyses can provide useful theoretical insights and thus facilitate the design of novel soft materials with improved fracture resistance.

On the other hand, direct experimental measurements of the crack tip deformation and stress fields are very limited at this time. For example, Livne et al. [24, 25] and Bouchbinder et al. [28] have measured the crack tip displacement field using digital image correlation. They first introduced image patterns to the crack tip region by means of shadowgraph and then determined the displacement field by correlating small elements in the reference and deformed images based on the introduced patterns. A similar method based on particle tracking was used in Chen et al. 
[93] to measure the crack tip velocity field for subsonic and supersonic dynamic cracks. There are at least two aspects in the experiments that can be further improved. First, the existing methods can only measure displacement field on the surface of thin sheet samples subjected to plane stress loading. No experimental data is available for plane strain or 3D cracks in soft materials since it is difficult to measure displacements in the bulk material. Second, full-field measurement of displacement and strain in the fully nonlinear zone around the crack tip is still not available. This may require accurate optical microscopy and image tracking in a $10 \mu \mathrm{m} \sim 1 \mathrm{~mm}$ region around the crack tip. Experimental efforts in this area are sorely needed to advance fundamental understanding in the fracture of soft materials.

\section{Acknowledgement}

C.Y.H. acknowledges the support of the U.S. Department of Energy, Office of Basic Energy Sciences, Division of Materials Sciences and Engineering under Award DE-FG02-07ER46463. R.L. acknowledges the start-up support from University of Colorado Boulder.

\section{References}

[1] Rivlin, R.S.; Thomas, A.G.; 1953, "Rupture of rubber. 1. Characteristic energy for tearing”, J. Polym. Sci., 10, 291-318.

[2] Thomas, A.G.; 1955, "Rupture of rubber. 2. The strain concentration at an incision", J. Polym. Sci., 18, 177-188.

[3] Greensmith, H.W.; Thoams, A.G.; 1955, "Rupture of rubber. 3. Determination of tear properties" J. Polym. Sci., 18, 189-200.

[4] Greensmith, H.W.; 1960, "Rupture of rubber”, Trans. Soc. Rheology, 4, 179-189.

[5] Lake, G.J.; Thomas, A.G.; 1967, "Strength of highly elastic materials", Proc. R. Soc. A, 300, 108-119.

[6] Gent, A.N.; 1996, “Adhesion and strength of viscoelastic solids. Is there a relationship between adhesion and bulk properties", Langmuir, 12, 4492-4496.

[7] Gong, J.P.; Katsuyama, Y.; Kurokawa, T.; Osada, Y.; 2003 "Double-network hydrogels with extremely high mechanical strength", Advanced Materials, 15, 1155-1158.

[8] Sun, J.Y.; Zhao, X.; Illeperuma, W.R.; Chaudhuri, O; Oh, K.H.; Mooney, D.J.; Vlassak, J.J.; Suo, Z.; 2012 "Highly stretchable and tough hydrogels", Nature, 489, 133-136. 
[9] Sun, T.L.; Kurokawa, T.; Kuroda, S; Ihsan, A.B.; Akasaki, I.; Sato, K.; Haque, M.A.; Nakajima, T.; Gong, J.P.; 2013, "Physical hydrogels composed of polyampholytes demonstrate high toughness and viscoelasticity”, Nature Materials, 12, 932-937.

[10] Henderson, K.J.; Zhou, T.C.; Otim, K.J.; Shull, K.R.; 2010 “Ionically cross-linked triblock copolymer hydrogels with high strength”, Macromolecules 43, 6193-6201.

[11] Zhao, X.; 2014, "Multi-scale multi-mechanism design of tough hydrogels: building dissipation into stretchy networks", Soft Matter, 10, 672-687.

[12] Ducrot, E.; Chen, Y.; Bulters M.; Sijbesma, R.P.; Creton, C.; 2014, “Toughening elastomers with sacrificial bonds and watching them break”, Science, 344, 186-189.

[13] Peppas, N.A.; Sahlin, J.J.; 1996, "Hydrogels as mucoadhesive and bioadhesive materials: a reivew”, Biomaterials, 17, 1553-1561.

[14] Yasuda, K.; Gong, J.P.; Katsuyama, Y.; Nakayama, A.; Tanabe, Y.; Kondo, E.; Ueno, M.; Osada Y.; 2005, "Biomechanical properties of high-toughness double network hydrogels", Biomaterials, 26, 4468-4475.

[15] Lee, K.Y.; Mooney, D.J.; 2001, "Hydrogels for tissue engineering”, Chemical Reviews, 101, 1869-1879.

[16] Calvert, P.; 2009, "Hydrogels for soft machines”, Advanced Materials, 21, 743-756.

[17] Gent, A.N.; Lindley, P.B.; 1959, "Internal rupture of bonded rubber cylinders in tension", Proc. R. Soc. A, 249, 195-205.

[18] Gent, A.N.; Wang, C.; 1991, "Fracture mechanics and cavitation in rubber-like solids", J. Mater. Sci., 26, 3292-3295.

[19] Cristiano, A.; Marcellan, A.; Long, R.; Hui, C.Y.; Stolk, J.; Creton, C.; 2010, “An experimental investigation of fracture by cavitation of model elastomeric networks", 48, 1409-1422.

[20] Hui, C.Y.; Jagota, A.; Bennison, S.J.; London, D.J.; 2003, "Crack blunting and the strength of soft elastic solids", Proc. R. Soc. A, 459, 1489-1516.

[21] Wang, X.; Hong, W.; 2012, "Delayed fracture in gels", Soft Matter, 8, 8171-8178.

[22] Hui, C.Y.; Long, R.; Ning, J.; 2013, "Stress relaxation near the tip of a stationary Mode I crack in a poroelastic solid", J. Appl. Mech., 80, 021014.

[23] Zehnder, A.T.; 2012 Fracture Mechanics, Springer, New York.

[24] Livne, A.; Bouchbinder, E.; Fineberg, J.; 2008, "Breakdown of linear elastic fracture mechanics near the tip of a rapid crack", Physical Review Letters, 101, 264301.

[25] Livne, A.; Bouchbinder, E.; Svetlizky I.; Fineberg, J.; 2010, “The near-tip fields of fast cracks", Science, 327, 1359-1363.

[26] Bouchbinder, E.; Livne, A.; Fineberg, J.; 2008, "Weakly nonlinear theory of dynamic fracture”, Physical Review Letters, 101, 264302. 
[27] Bouchbinder, E.; Liven, A.; Fineberg, J.; 2009, "The 1/r singularity in weakly nonlinear fracture mechanics”, J. Mech. Phys. Solids, 57, 1568-1577.

[28] Bouchbinder, E.; Livne A.; Fineberg J.; 2010, "Weakly nonlinear fracture mechanics: experiments and theory", Int. J. Fracture, 162, 3-20.

[29] Lefranc, M.; Bouchaud, E.; 2014, "Mode I fracture of a biopolymer gel: Rate-dependent dissipation and large deformations disentangled", Extreme Mechanics Letters, 1, 97-103.

[30] Wong, F.S.; Sheild, R.T.; 1969, "Large plane deformation of thin elastic sheets of neoHookean materials", Z. Angew. Math. Phys., 20, 176-199.

[31] Knowles, J.K.; Sternberg, E.; 1973, “An asymptotic finite deformation analysis of the elastostatic field near the tip of a crack", J. Elasticity, 3, 67-107.

[32] Knowles, J.K.; Sternberg, E.; 1974, "Finite deformation analysis of the elastostatic field near the tip of a crack: reconstruction and high-order results", J. Elasticity 4, 201-233.

[33] Rice, J.R.; 1968b, "Mathematical analysis in the mechanics of fracture", in Fracture: an advanced treatise, edited by H. Liebowitz, Academic Press, Vol. 2, 191-311.

[34] Fowler, G.F.; 1984, "Finite plane and anti-plane elastostatic fields with discontinuous deformation gradients near the tip of a crack", J. Elasticity 14, 287, 328.

[35] Gao, Y.C.; 1990, “Elastostatic crack tip behavior for a rubber-like material”, Theor. Appl. Fract. Mech. 14, 219-231.

[36] Le, K.C., 1992, "On the singular elastostatic field induced by a crack in a Hadamard material”, Q. J. Mech. Appl. Math. 45, 101-117.

[37] Le, K.C., Stumpf, H., 1993, "The singular elastostatic field due to a crack in rubberlike materials", J. Elasticity, 32, 203-241.

[38] Gao, Y.C. ; 1997, "Large deformation field near a crack tip in rubber-like material", Theor. Appl. Fract. Mech. 26, 155-162.

[39] Stephenson, R.A.; 1982, "The equilibrium field near the tip of a crack for finite plane strain of incompressible elastic materials", J. Elasticity 12, 65-99.

[40] Abeyaratne, R.; Yang, J.S.; 1987, "Localized shear deformations near the tip of a Mode-I crack", J. Elasticity 17, 93-112.

[41] Hao, T.H.; 1990, "Near field behavior of in-plane crack extension in nonlinear incompressible materials", Theor. Appl. Fract. Mech. 12, 241-249.

[42] Gao, Y.C., Durban, D., 1995, “The crack tip field in a rubber sheet”, Eur. J. Mech. A/Solids, 14, 665-677.

[43] Tarantino, A.M., 1996, "Thin hyperelastic sheets of compressible material: field equations, airy stress function and an application in fracture mechanics”, J. Elast. 44, 37-59.

[44] Gao, Y.C.; Chen, S.H.; 2001, "Large strain field near a crack tip in a rubber sheet", Mech. Res. Commun., 28, 71-78. 
[45] Geubelle, P.H.; Knauss, W.G.; 1994a, "Finite strain at the tip of a crack in a sheet of hyperelastic materials: I. Homogeneous case", J. Elasticity, 35, 61-98.

[46] Long, R.; Krishnan, V.R.; Hui, C.Y.; 2011, "Finite strain analysis of crack tip fields in incompressible hyperelastic solids loaded in plane stress", J. Mech. Phys. Solids, 59, 672695.

[47] Knowles, J.K., 1977, "The finite anti-plane shear field near the tip of a crack for a class of incompressible elastic solids", Int. J. Fract., 13, 611-639.

[48] Long, R.; Hui, C.Y.; 2011, "Effects of finite chain extensibility on the stress fields near the tip of a mode III crack", Proc. Roy. Soc. A, 467, 3170-3187.

[49] Knowles, J.K.; Sternberg, E.; 1983, "Large deformations near a tip of an interface-crack between two neo-Hookean sheets", J. Elasticity, 13, 257-293.

[50] Geubelle, P.H.; Knauss, W.G.; 1994, "Finite strain at the tip of a crack in a sheet of hyperelastic material: II. Special biomaterial cases”, J. Elasticity, 35, 99-137.

[51] Geubelle, P.H.; Knauss, W.G.; 1994, "Finite strain at the tip of a crack in a sheet of hyperelastic material: II. General biomaterial cases”, J. Elasticity, 35, 139-174.

[52] Herrmann, J.M.; 1989, "An asymptotic analysis of finite deformation near the tip of an interface crack", J. Elasticity, 21, 227-269.

[53] Herrmann, J.M.; 1992, "An asymptotic analysis of finite deformation near the tip of an interface crack: part II”, J. Elasticity, 29, 203-241.

[54] Ru, C.Q.; 1997, "Finite strain singular field near the tip of a crack terminating at a material interface", Math. Mech. Solids, 2, 49-73.

[55] Ru, C.Q.; 2002, “On complex-variable formulation for finite plane elastostatics of harmonic materials", Acta Mech., 156, 219-234.

[56] Gao, Y.C.; Jin, M.; Dui, G.S., 2008, "Stresses, singularities, and a complementary energy principle for large strain elasticity”, Applied Mechanics Reviews, 61, 030801.

[57] Ravichandran, G.; Knauss, W.G.; 1989, “A finite elastostatic analysis of bimaterial interface cracks", Int. J. Fracture, 39, 235-253.

[58] Lund, R.A.; Westmann, R.A.; 1990, "Finite element analysis of hyperelastic large deformation crack tip fields", Int. J. Fract. 43, 257-270.

[59] Batra, R.C.; Zhang, J.P.; 1993, “Analysis of deformations near a crack tip in a compressible nonlinear elastic material", Eng. Fract., Mech. 40, 413-432.

[60] Quigley, C.J.; Parks, D.M.; 1994, “The finite deformation field surrounding a mode I plane strain crack in a hyperelastic incompressible material under small-scale nonlinearity", Int. J. Fracture, 65, 75-96. 
[61] Legrain, G.; Moes, N.; Verron, E.; 2005, "Stress analysis around crack tips in finite strain problems using the extended finite element method", Int. J. Numer, Methods Eng. 63, 290314.

[62] Krishnan, V.R.; Hui, C.Y.; Long, R.; 2008, "Finite strain crack tip fields in soft incompressible elastic solids", Langmuir, 24, 14245-14253.

[63] Krishnan, V.R.; Hui, C.Y.; 2009, "Finite strain stress fields near the tip of an interface crack between a soft incompressible elastic material and a rigid substrate", Eur. Phys. J. E, 29, 61-72.

[64] Seitz, M.E.; Martina, D.; Baumberger, T.; Krishnan, V.R.; Hui, C.Y.; Shull, K.R.; 2009, "Fracture and large strain behavior of self-assembled triblock copolymer", Soft Matter, 2, 447-456.

[65] Gent, A.N.; 1996, “A new constitutive relation for rubber”, Rubber Chem. Technol., 69, 59-61.

[66] Arruda, E.M.; Boyce, M.C.; 1993, "A three dimensional constitutive model for the large stretch behavior of rubber elastic materials", J. Mech. Phys. Solids, 41, 389-412.

[67] Boyce, M.C.; Arruda, E.M.; 2000, "Constitutive models of rubber elasticity: a review", Rubber Chemistry and Technology, 73, 504-523.

[68] Williams, M., 1957, "On the stress distribution at the base of a stationary crack", J. Appl. Mech., 24, 109-114.

[69] Neuber, H.; 1961, "Theory of stress concentration for shear-strain prismatical bodies with arbitrary non-linear stress strain law”, J. Appl. Mech., 28, 544-550.

[70] Rice, J.R.; 1967, "Stresses due to a sharp notch in a work-hardening elastic-plastic material loaded by longitudinal shear", J. Appl. Mech., 34, 287-298.

[71] Goldman Boue, T., Harpaz, R., Fineberg, J., Bouchbinder, E.; 2015, "Failing softly, a fracture theory of highly-deformable materials", Soft Matter, 11, 3812-3821.

[72] Rashetnia, R., Mohammadi, S., 2015, "Finite strain fracture analysis using the extended finite element method with new set of enrichment functions", Int. J. Numer., Meth. Engng., 102, 1316-1351.

[73] Harpaz, R.; Bouchbinder, E.; 2012, "A nonlinear symmetry breaking effect in shear cracks”, J. Mech. Phys. Solids, 60, 1703-1709.

[74] Hui, C.Y.; Ruina, A.; 1995, "Why K? High order singularities and small scale yielding", Int. J. Fracture, 72, 97-120.

[75] Lengyel, T.H.; Long, R.; Schiavone, P.; 2014, "Effect of interfacial slippage on the near-tip fields of an interface crack between a soft elastomer and a rigid substrate", Proc. R. Soc. A, 470, 20140497. 
[76] Nase, J.; Creton, C.; Ramos, O.; Sonnenberg, L.; Yamaguchi, T.; Lindner, A.; 2010, "Measurement of the receding contact angle at the interface between a viscoelastic material and a rigid surface", Soft Matter, 6, 2685-2691.

[77] Newby, B.Z.; Chaudhury, M.K.; Brown, H.R.; 1995, "Macroscopic evidence of the effect of interfacial slippage on adhesion”, Science, 269, 1407-1409.

[78] Trabelsi, S.; Albouy, P.A.; Rault, J.; 2002, "Stress-induced crystallization around a crack tip in natural rubber", Macromolecules, 35, 10054-10061.

[79] Eshelby, J.D.; 1969, "Energy relations and the energy-ommentum tensor in continuum mechanics", in Inelastic behavior of Solids, edited by M.F. Kanninen, W.F. Adler, A.R. Rosenfield, R.I. Jaffee, McGraw-Hill, New York, 77-115.

[80] Rice, J.R.; 1968, “A path independent integral and the approximate analysis of strain concentration by notches and cracks", J. Appl. Mech., 35, 379-386.

[81] Baumberger, T.; Ronsin, O.; 2010, "A convective instability mechanism for quasistatic crack branching in a hydrogel”, Eur. Phys. J. E, 31, 51-58.

[82] Baumberger, T.; Caroli, C.; Martina, D.; Ronsin O.; 2008, "Magic angles and crosshatching instability in hydrogel fracture”, Phys. Rev. Lett., 100, 178303.

[83] Ronsin, O.; Caroli, C.; Baumberger, T.; 2014, "Crack front echelon instability in mixed mode fracture of a strongly nonlinear elastic solid”, Eur. Phys. Lett., 105, 34001.

[84] Ghatak, A.; Chaudhury, M.K.; 2003, “Adhesion-induced instability patterns in thin controlled elastic film”, Langmuir, 19, 2621-2631.

[85] Adda-Bedi, M.; Mahadevan, L.; 2006, "Crack-front instability in a confined elastic film”, Proc. R. Soc. A 462, 3233-3251.

[86] Holzapfel, G.A.; 2000, Nonlinear solid mechanics: a continuum approach for engineering. John Wiley \& Sons, London, UK.

[87] Fung, Y.C.; 1993, Biomechanics: Mechanical properties of living tissues, 2nd edition, Springer, New York.

[88] Baumberger, T.; Caroli, C.; Martina, D.; 2006, "Solvent control of crack dynamics in a reversible hydrogel", Nature Materials, 5, 552-555.

[89] Deegan, R.D.; Petersan P.J.; Marder, M.; Swinney, H.L.; 2002, “Oscillating fracture paths in rubber", Phys. Rev. Lett. 88, 014304.

[90] Petersan, P.J.; Deegan, R.D.; Marder, M.; Swinney, H.L.; 2004, “Cracks in rubber under tension exceed the shear wave speed”, Phys. Reve. Lett., 93, 015504.

[91] Marder, M.; 2006, “Supersonic rupture of rubber”, J. Mech. Phys. Solids, 54, 491-532.

[92] Bouchbinder, E.; Procaccia, I.; 2007, “Oscillatory instability in two-dimensional dynamic fracture", Phys. Rev. Lett. 98, 124302. 
[93] Chen, C.H.; Zhang, H.P.; Niemczura, J.; Ravi-Chandar, K.; Marder, M.; 2011, "Scaling of crack propagation in rubber sheets", Eur. Phys. Lett., 96, 36009.

[94] Tarantino, A.M.; 1999, "On the finite motions generated by a Mode I propagating crack", J. Elasticity, 57, 85-103.

[95] Tarantino, A.M.; 2005, "Crack propagation in finite elastodynamics", Math. Mech. Solid., 10, 577-601.

[96] Blatz, P.J.; Ko, W.L.; 1962, “Application of finite elastic theory to the deformation of rubbery materials”, Trans. Soc. Rheol., 6, 223-252. 


\section{Figure Captions}

Figure 1 Schematics of crack geometry and loading conditions. (A) Undeformed configuration of an edge crack. $\left(X_{1}, X_{2}\right)$ denote the coordinates of a material point in undeformed configuration (B) Plane strain or plane stress deformation of the crack in A. $\left(y_{1}, y_{2}\right)$ denote the coordinates of a material point in the deformed configuration. Here we assume Mode-I condition where the crack opens symmetrically as an example for illustration. (C) Anti-plane shear (Mode III) deformation of the crack. The dot and cross symbols are used to represent out of plane loading either in terms of displacement or traction.

Figure 2 The dimensionless reduced stress $\sigma_{R}$ versus stretch ratio $\lambda$. The dashed lines represent generalized neo-Hookean (GNH) model with $b=1$ and $n=1,2$, or 3 . The solid line is given by the exponentially hardening model (EM) with $J_{n}=5$. The dash-dotted line is given by the Gent model (GM) with $J_{n}=5$.

Figure 3 (A) The function $U(\theta, n)$ versus $\theta$ for $n=0.7,1,2,3$ or 10. (B) The function $H(\theta, n)$ versus $\theta$ for $n=1.7,2,3$ or 10 . Note that $H(\theta, n)$ is defined only for $n>3 / 2$.

Figure 4 Crack opening profiles predicted by the asymptotic solution. Both the deformed coordinates $y_{1}$ and $y_{2}$ are normalized by $a^{2 n}$. (A) The cases where $1 / 2<n<3 / 2$ with $b_{0} / a^{2-2 n}=0.5$, 1,5 , or 10 . (B) The cases where $n>3 / 2$ with $n=2,3,5$, or 10 .

Figure 5 Schematic of crack deformation under mixed-model loading. The dashed lines are intended to show the undeformed configuration of the crack specimen.

Figure 6 The exponent of $r$ in the leading order term of $y_{1}$ as a function of $n$. For $n<n^{*} \approx 1.46$ the exponent is $d$. Numerical solution of $d$ is shown as the solid line and the approximate solution in (59) is shown as the dotted line. For $n>n^{*}$, the exponent is given by $1+1 /(4 n)$.

Figure 7 Numerical solutions of $g(\theta, n)$ for (A) $0.5<n<1.46$ and (B) $n>1.46$.

Figure 8 Schematic of the three regions around the crack tip in the undeformed configuration: Region I $(\pi>\theta>\pi / 2)$, Region II $(\pi / 2>\theta>-\pi / 2)$ and Region III $(-\pi / 2>\theta>-\pi)$. Adapted from Long and Hui [48]. Reproduced with permission from the Royal Society of London.

Figure 9 (A) Plot of $G(\theta)$ versus $\theta$ where $G_{0}=G(\theta=0)=1.75$ (dimensionless) in the particular plot. (B) Plot of $f(\theta)$ versus $\theta$. In both plots, $\theta$ ranges from 0 to $\pi / 2$ since $G(\theta)$ is an even function of $\theta$ and $f(\theta)$ is an odd function. The symbols are finite element data at two different radius $r / L_{0}=0.0004$ and 0.0002 where $L_{0}$ is a characteristic length scale of the finite element model. Adapted from Long et al. [46] and reproduced with permission from Elsevier.

Figure 10 (A) Schematic for the deformation of the interface crack. The region where the neartip asymptotic solution dominates is exaggerated for clear illustration. (B) Asymptotic solutions for the crack opening profiles for a plane stress interface crack between a neo-Hookean material 
and a rigid substrate. The deformed coordinates $y_{1}$ and $y_{2}$ are normalized by $a_{2}^{2}$, and $a_{1} / a_{2}=$ $-0.5,0,0.5,1$ or 2 .

Figure 11 Deformation of the crack tip region based on the plan strain Mode I solution in (89a,b) for neo-Hookean solid. The coordinates are normalized by $a^{2}$ and we have assumed $b_{0}=1$ and $c_{3}=1$. (A): Undeformed configuration and (B) Deformed configuration. The red element is used to illustrate how an individual element deforms.

Figure 12 Contour plots of the crack tip true stress components normalized by the shear modulus $\mu$. (A-C) $\tau_{11}, \tau_{12}$ and $\tau_{22}$ in the undeformed configuration; (D-F) $\tau_{11}, \tau_{12}$ and $\tau_{22}$ in the deformed configuration. The coordinates are normalized by $a^{2}$ and we have assumed $b_{0}=1$ and $c_{3}=1$. These plots are based on the plane strain Mode-I solution in (50) for neo-Hookean solid.

Figure 13 Deformation of the crack tip region based on the plane stress Mode I solution in (65) for neo-Hookean solid. The coordinates are normalized by $a^{2}$ and we have chosen $c=1.2$ according the finite element results of Krishnan et al. [62]. (A): Undeformed configuration and (B) Deformed configuration. The red element is used to highlight how an individual element deforms.

Figure 14 Contour plots of the crack tip true stress components normalized by the shear modulus $\mu$. (A-B) $\tau_{12}$ and $\tau_{22}$ in the undeformed configuration; (C-D) $\tau_{12}$ and $\tau_{22}$ in the deformed configuration. The coordinates are normalized by $a^{2}$ and we have assumed $c=1.2$. These plots are based on the plane stress Mode-I solution in (68) for neo-Hookean solid. The component $\tau_{11}$ is not plotted since its first order term is constant.

Figure 15 Schematic of the contour for J-integral. The contour is shown as a dashed curve and $N=N_{\alpha} \vec{e}_{\alpha}$ is the unit outward normal vector of the contour.

Figure 16 Schematic of the pure shear specimen for facture tests. 

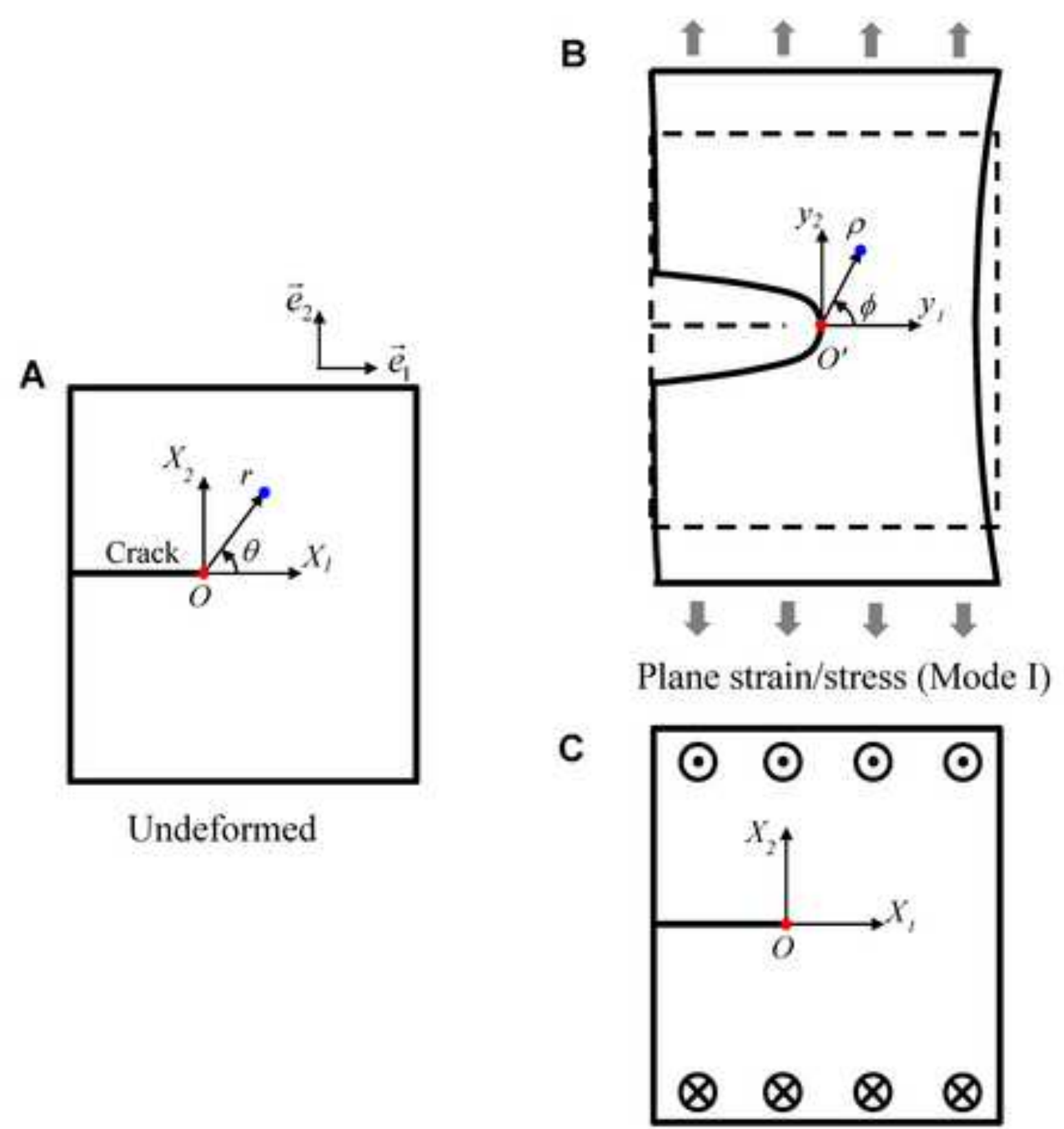

Anti-plane shear 


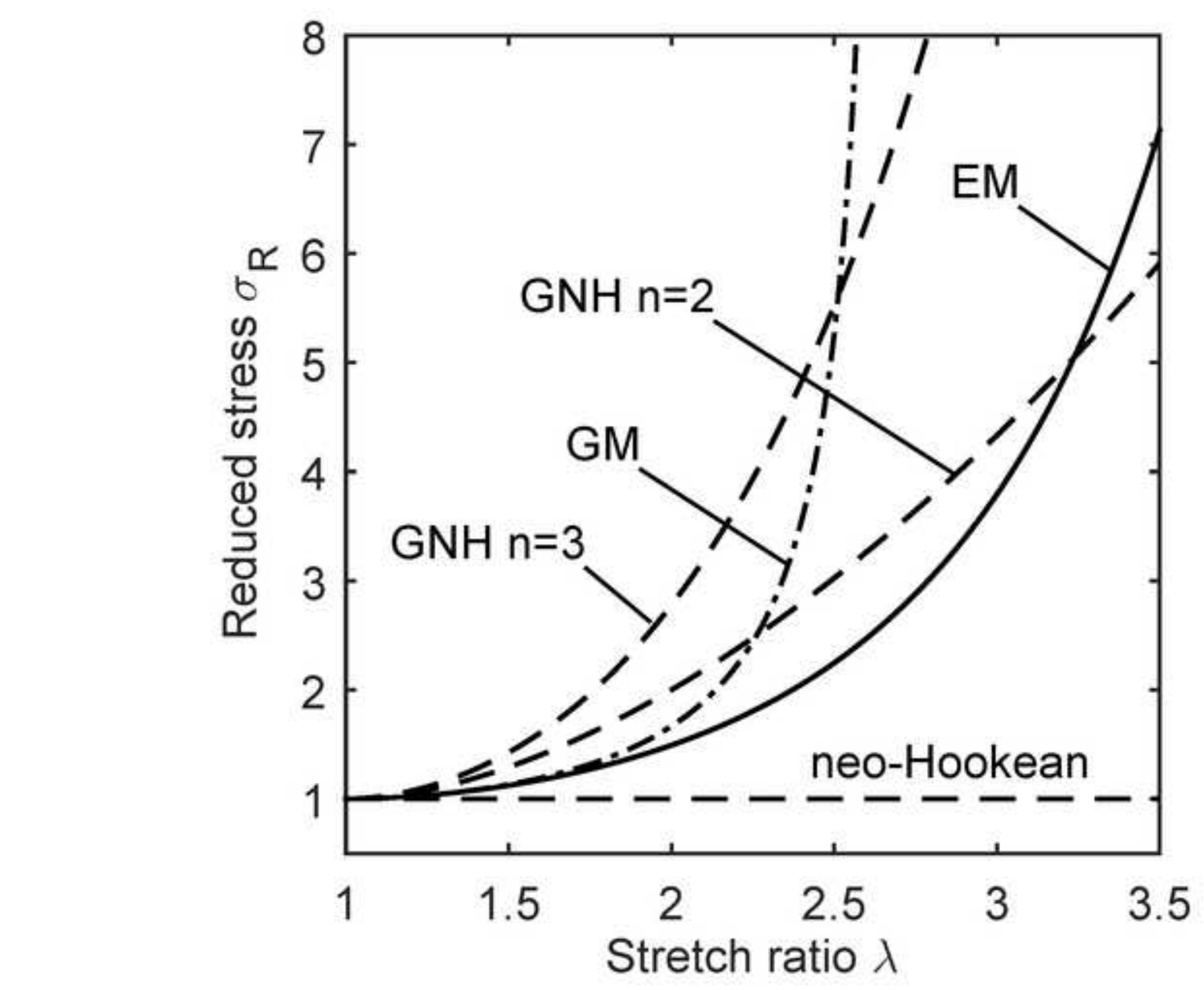



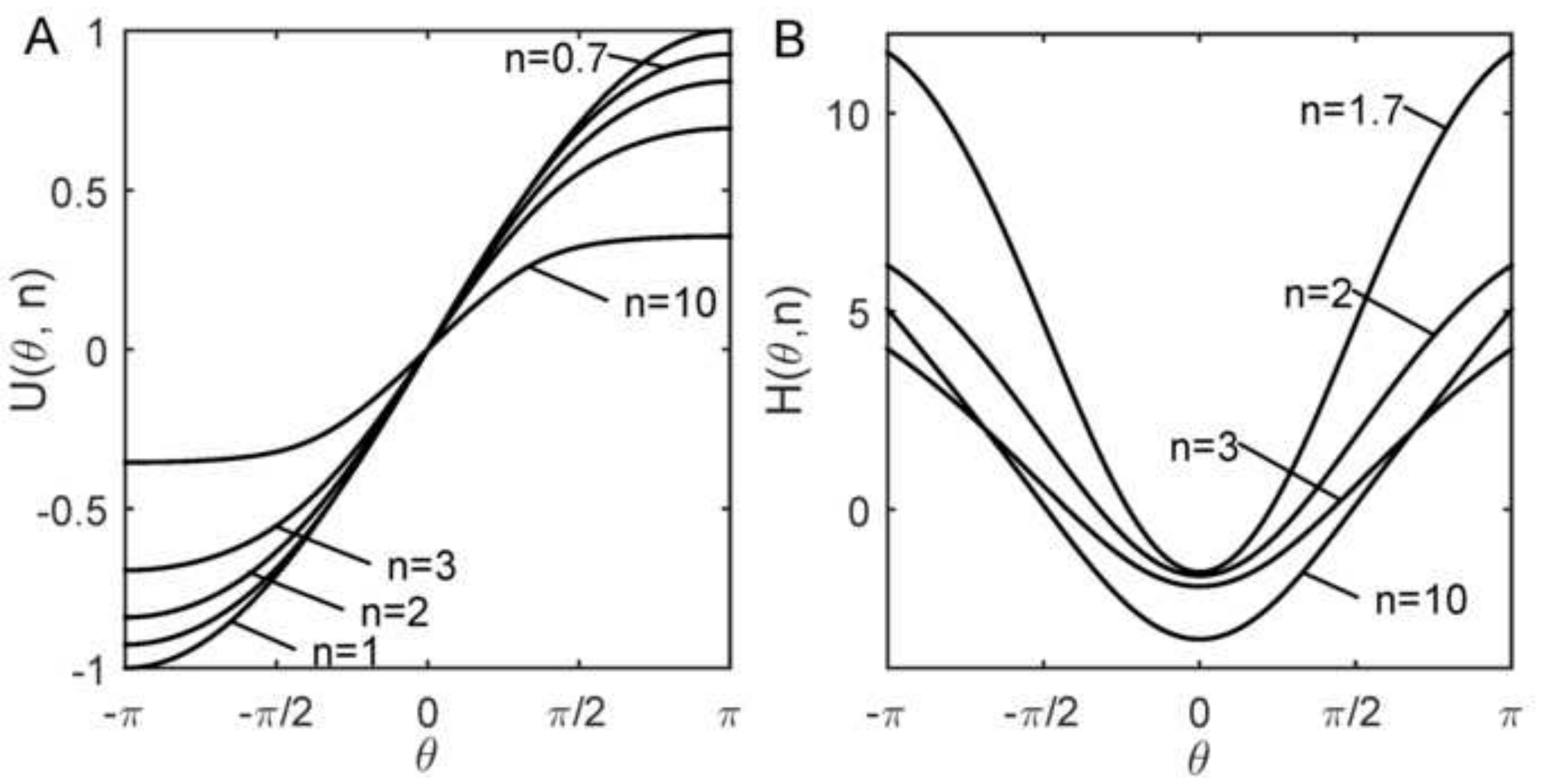

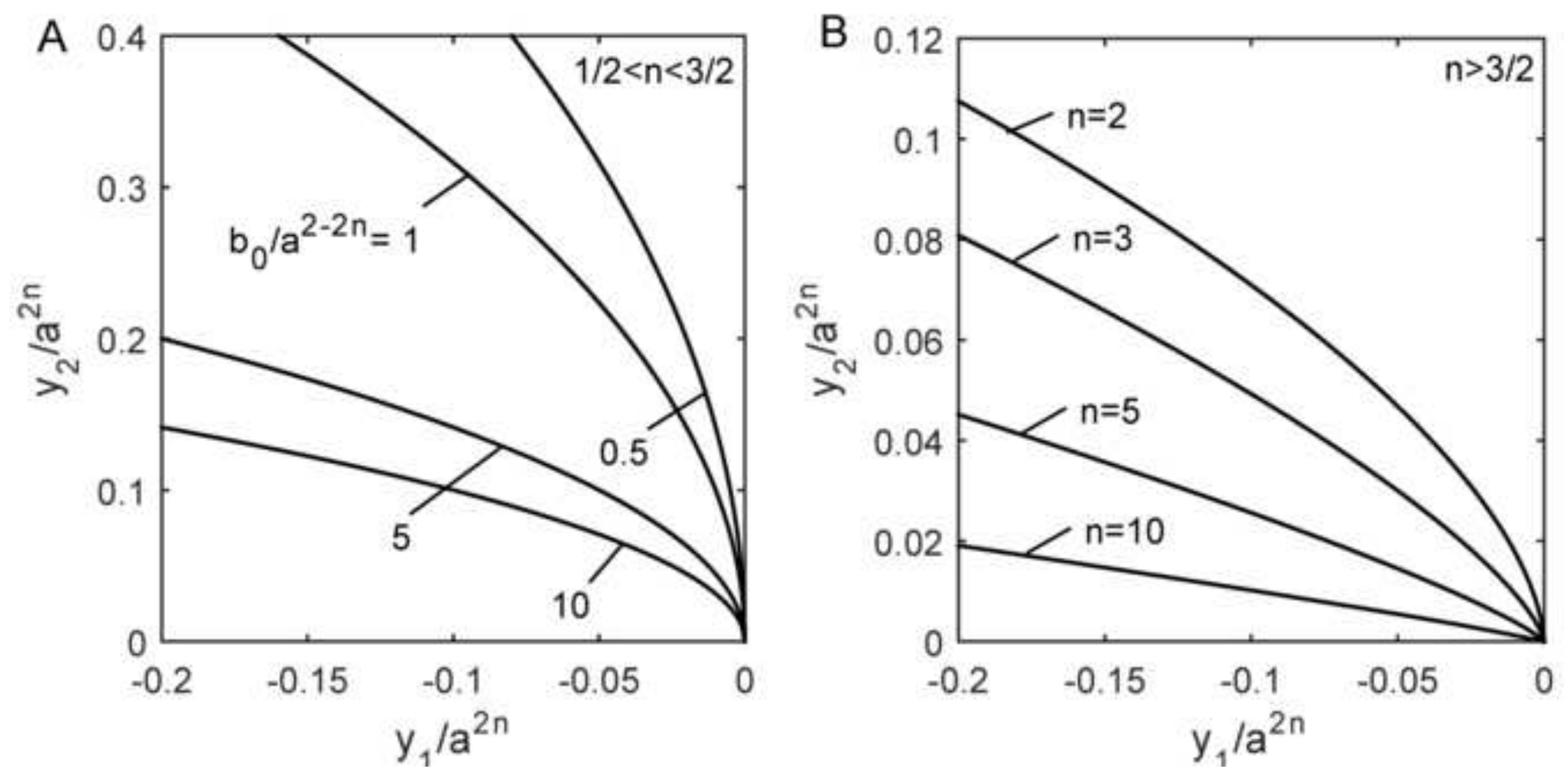

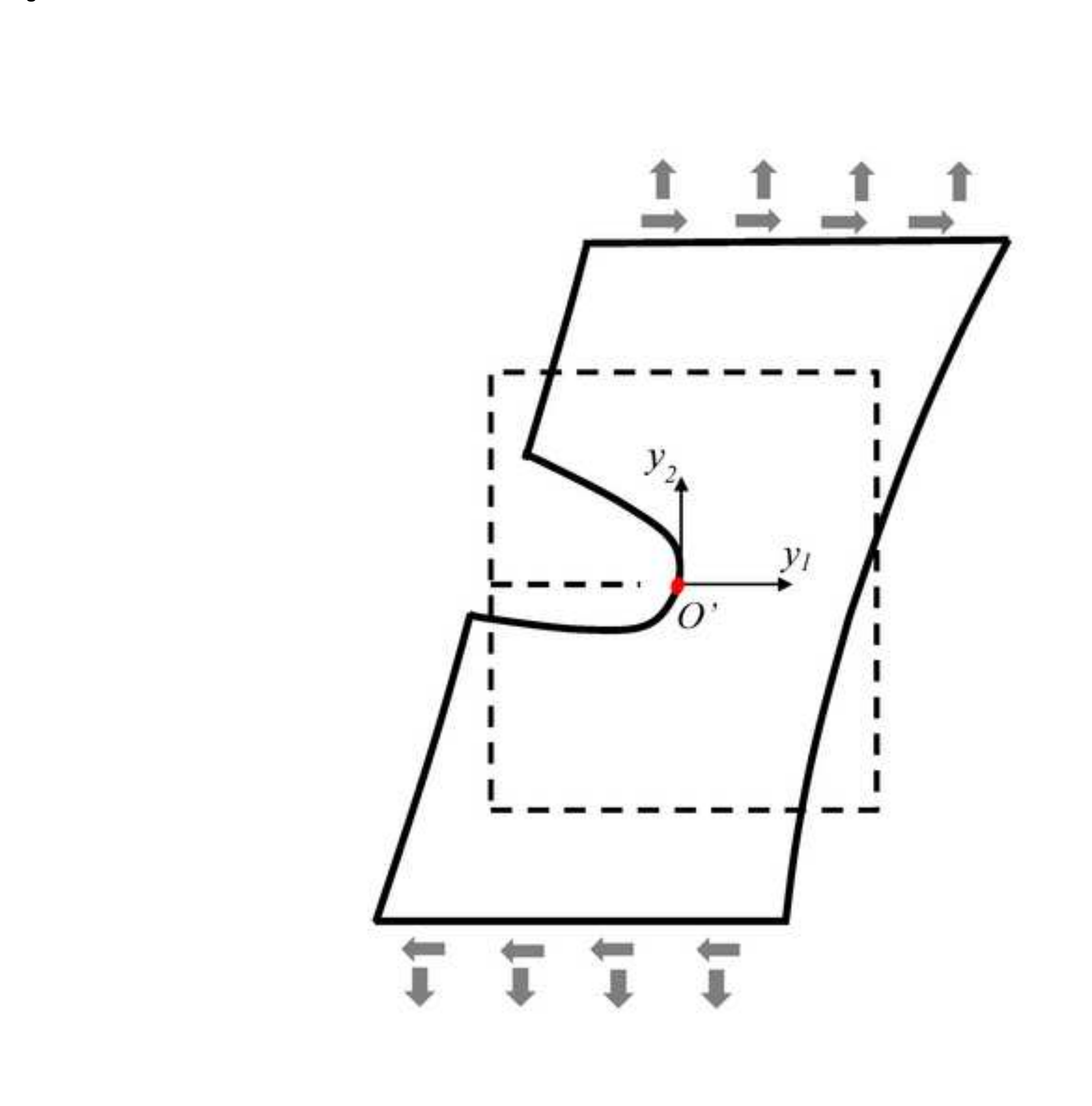

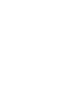
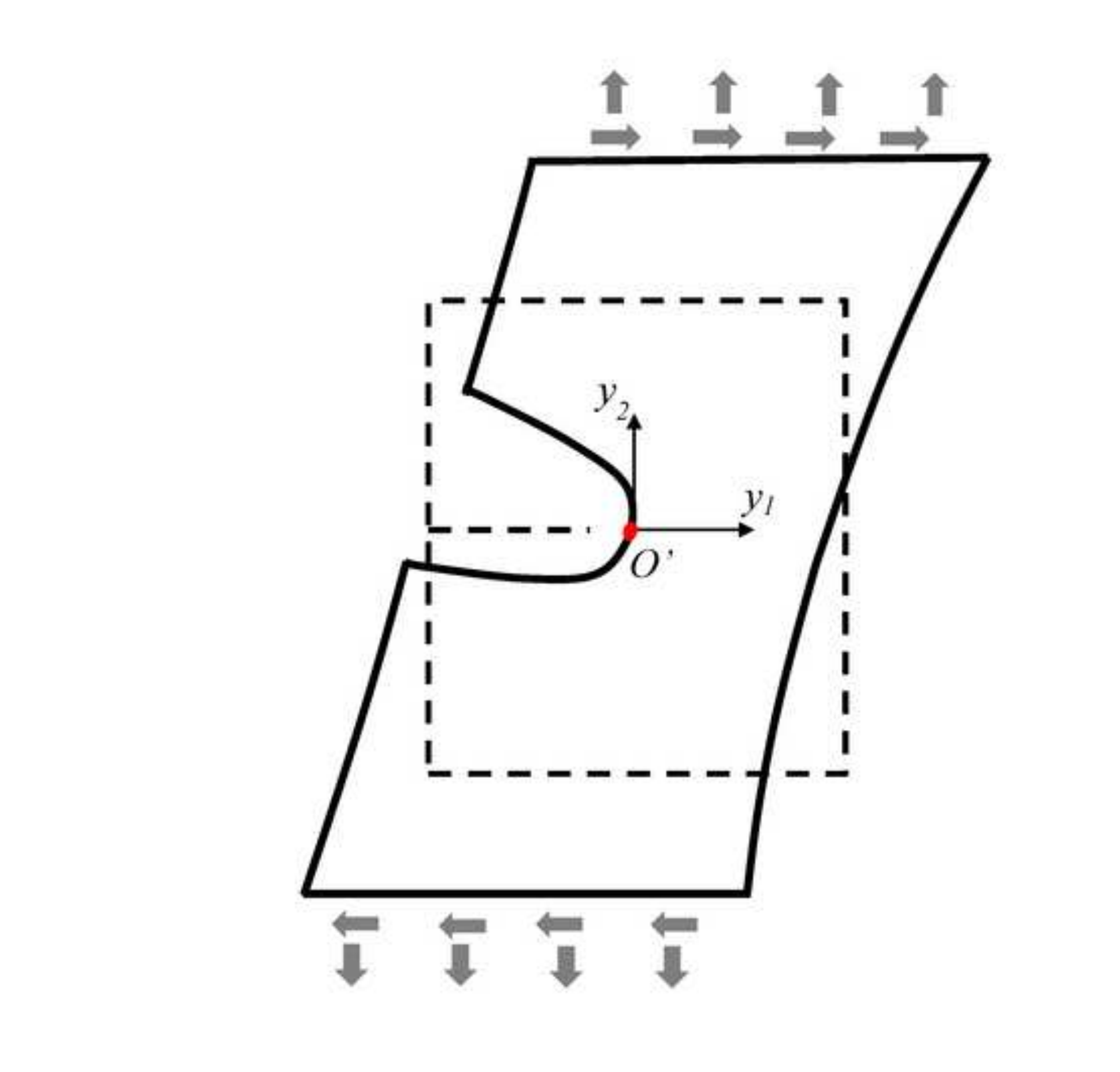

.

Figure




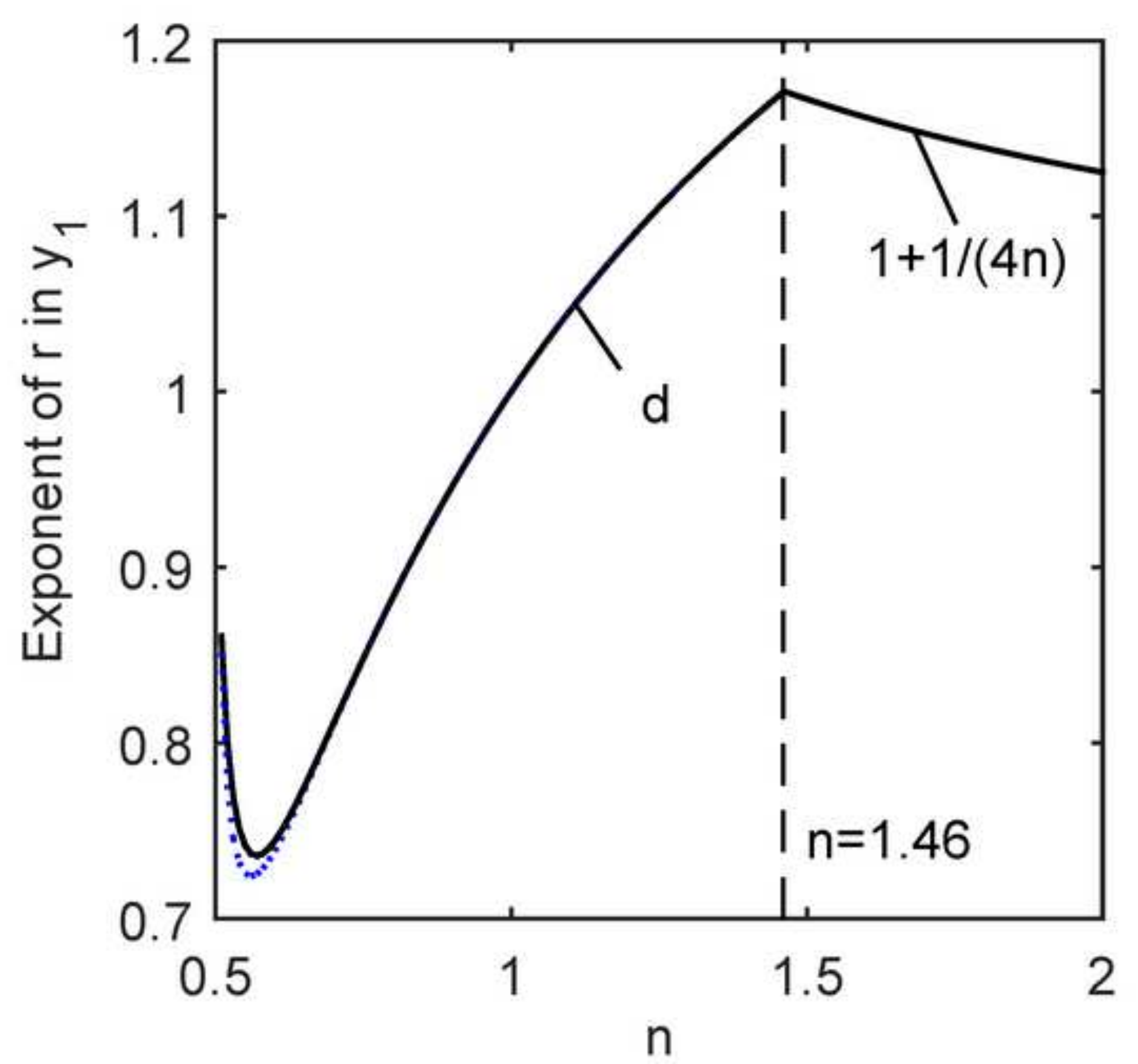

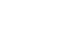



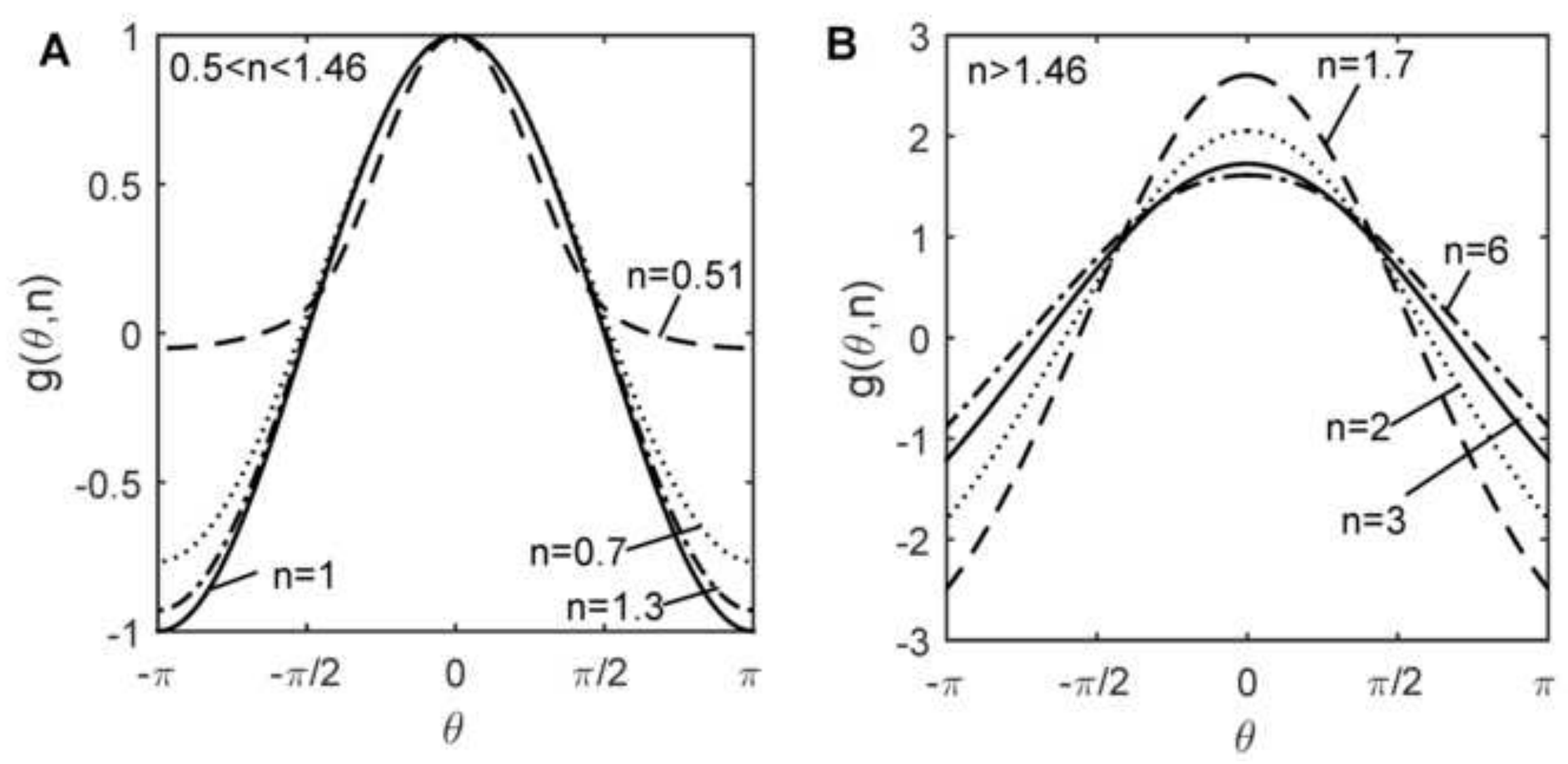


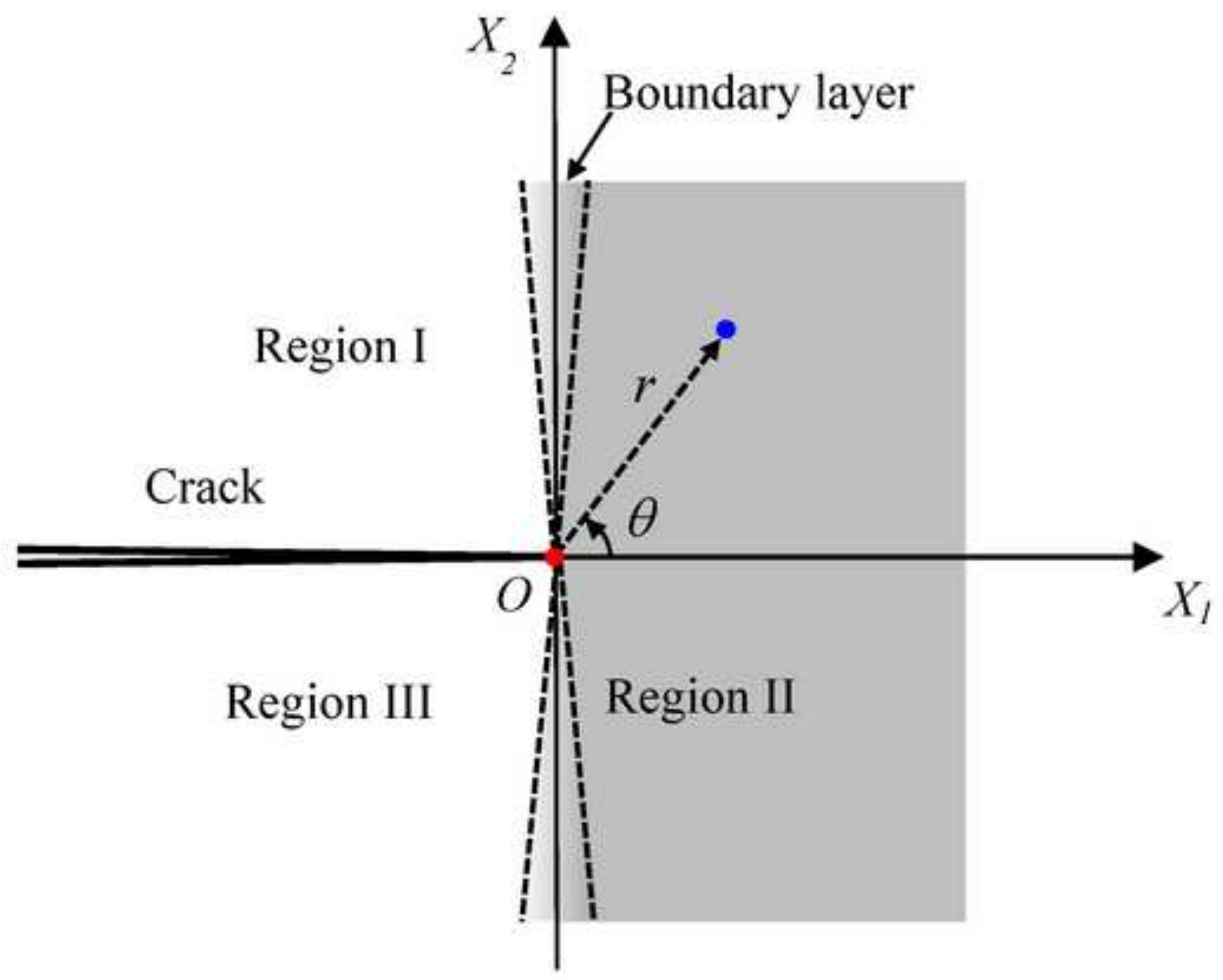

Crack

Region III 
A

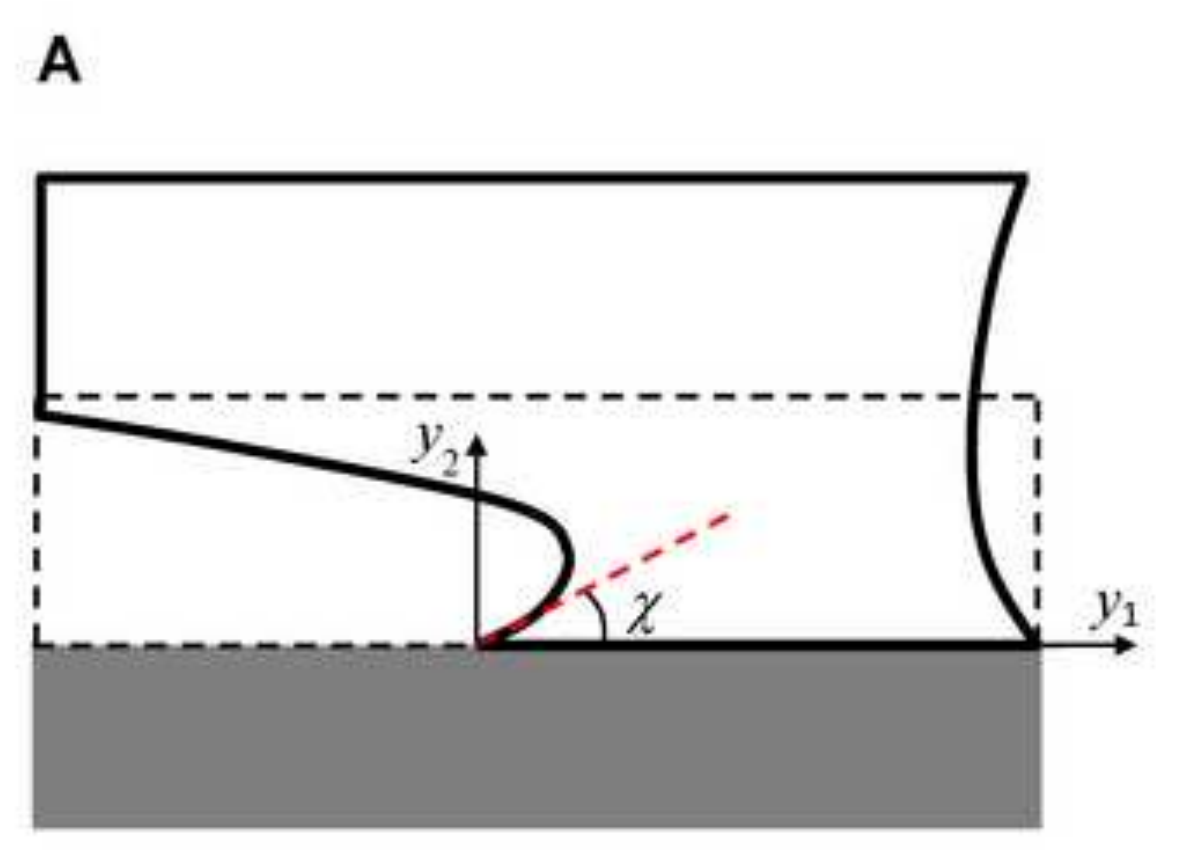

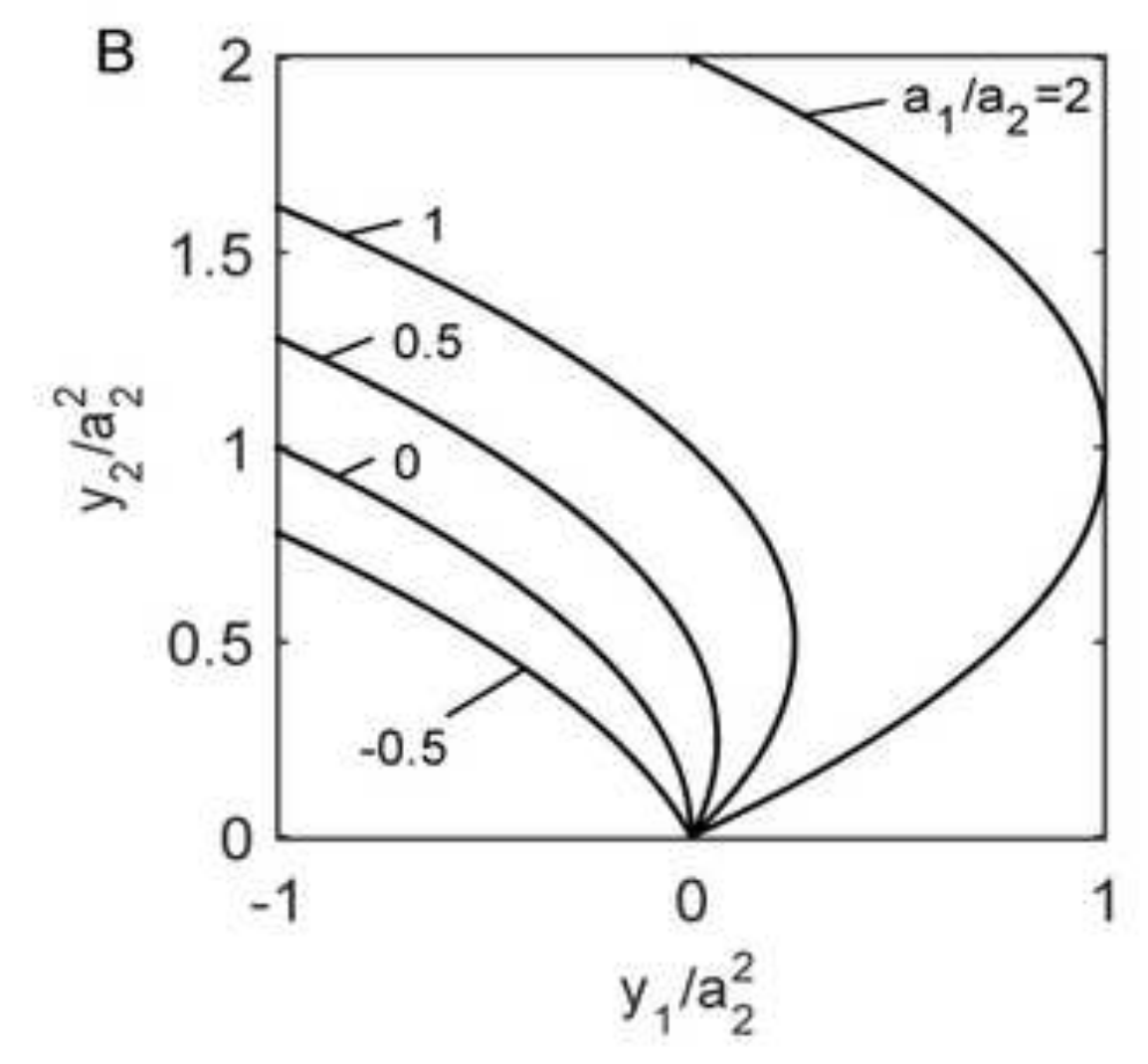



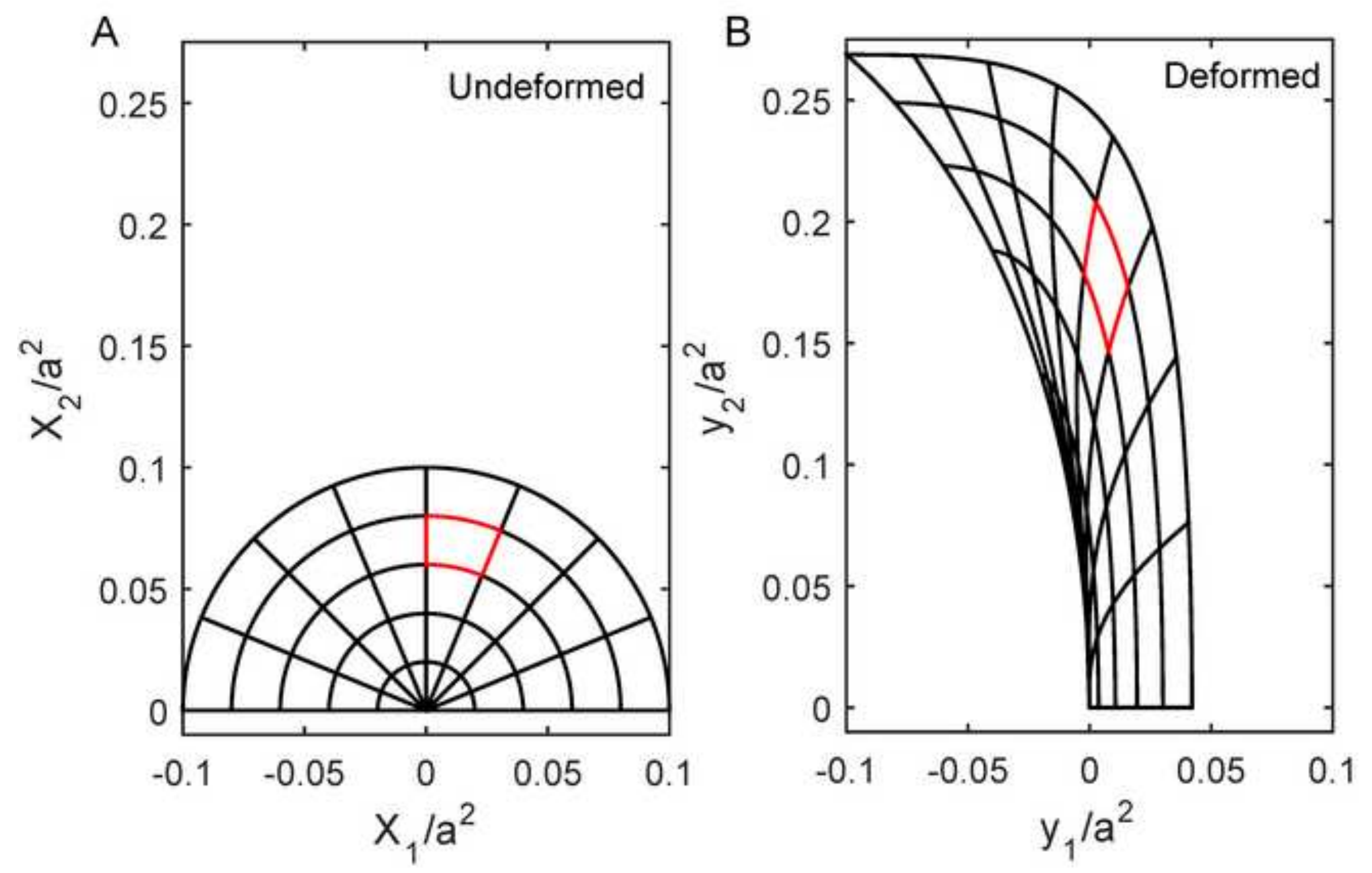

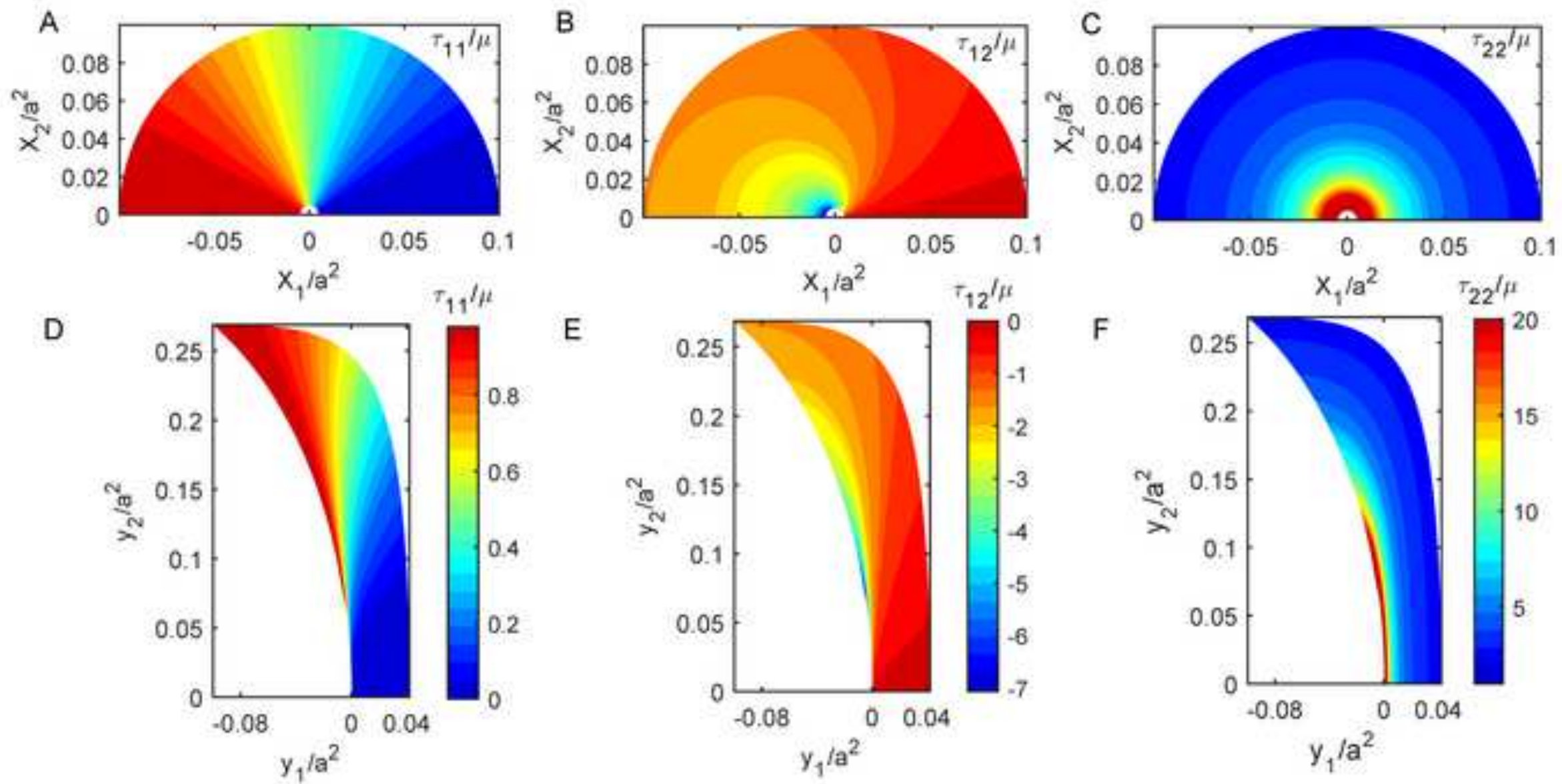

D

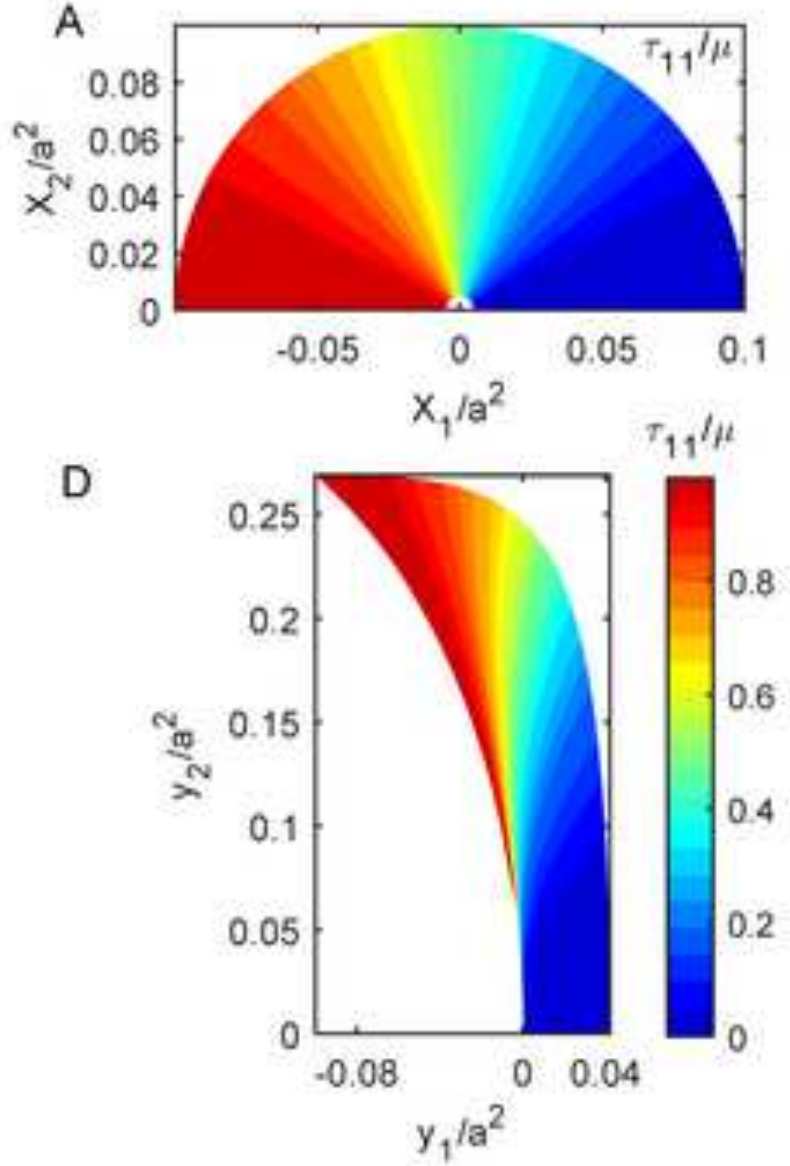

E

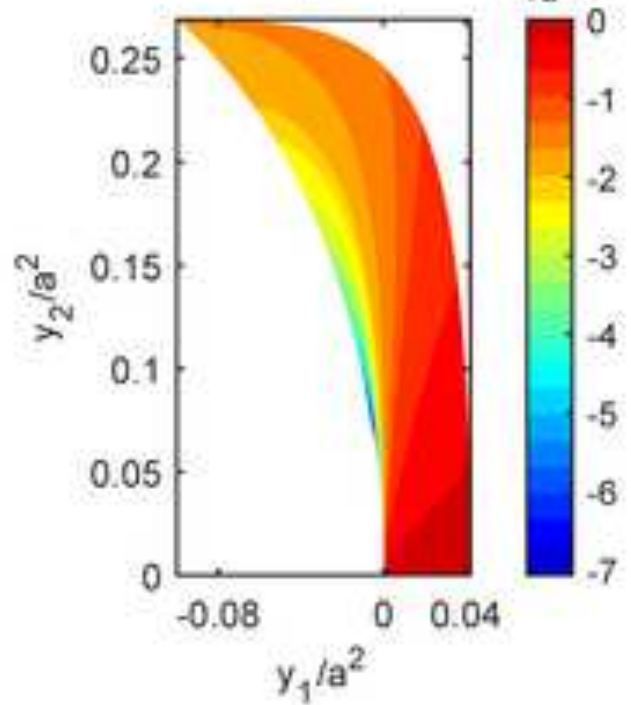

F

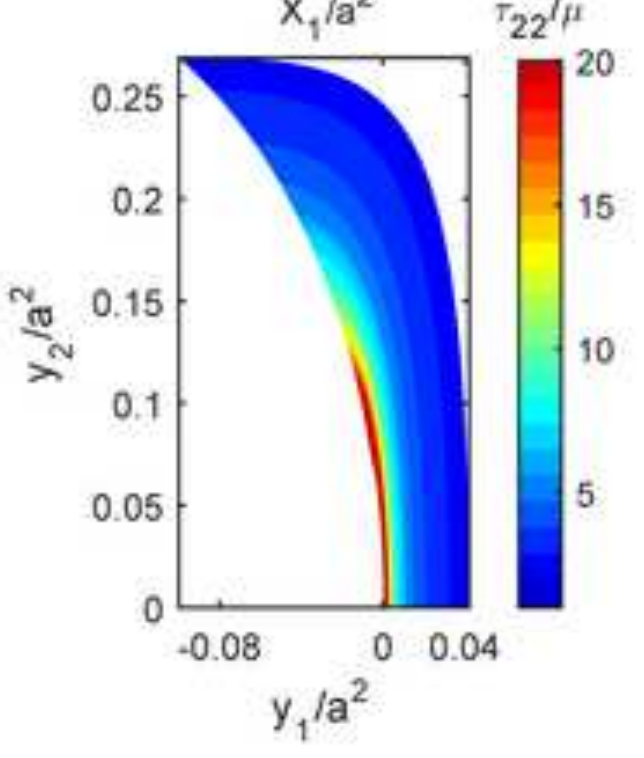



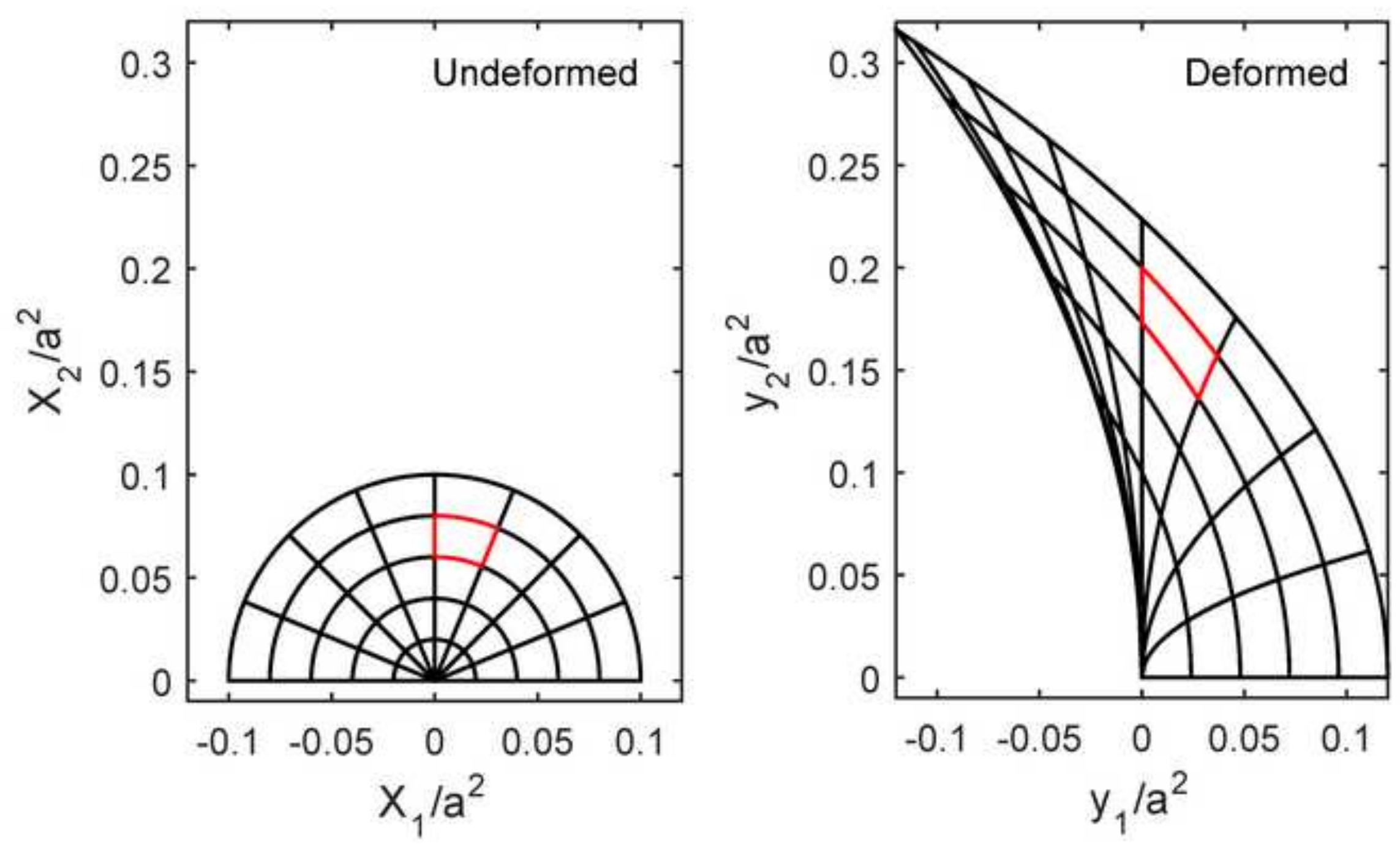


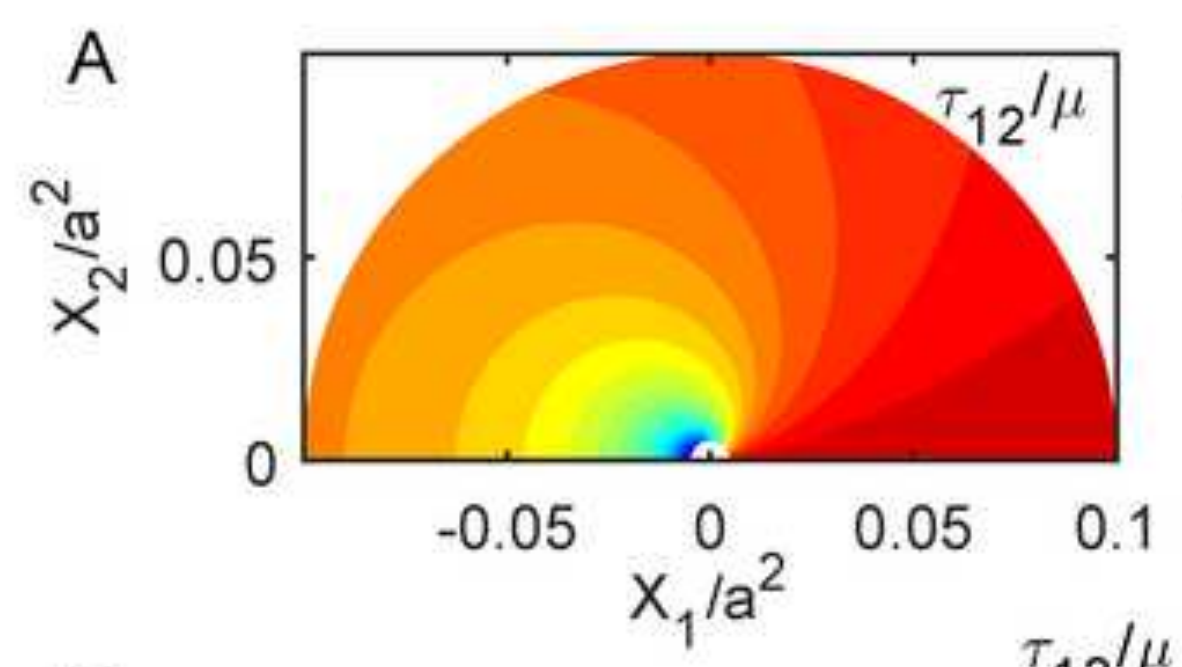

C

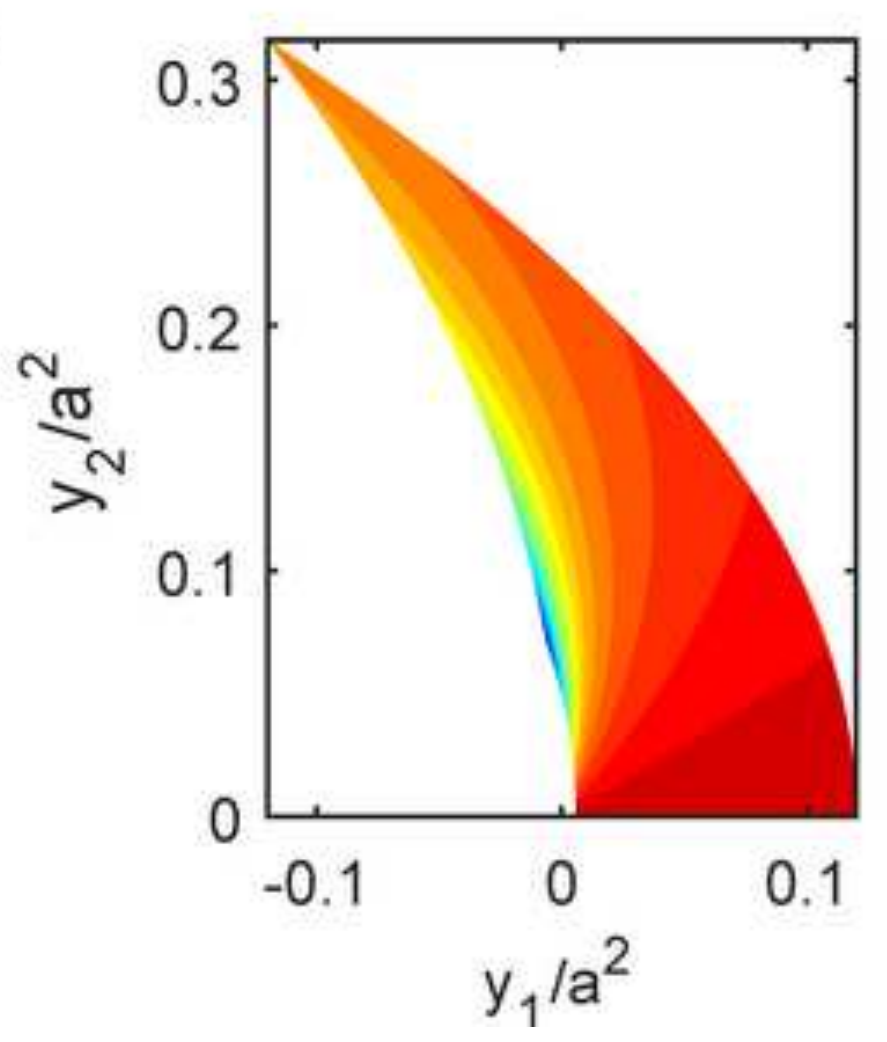

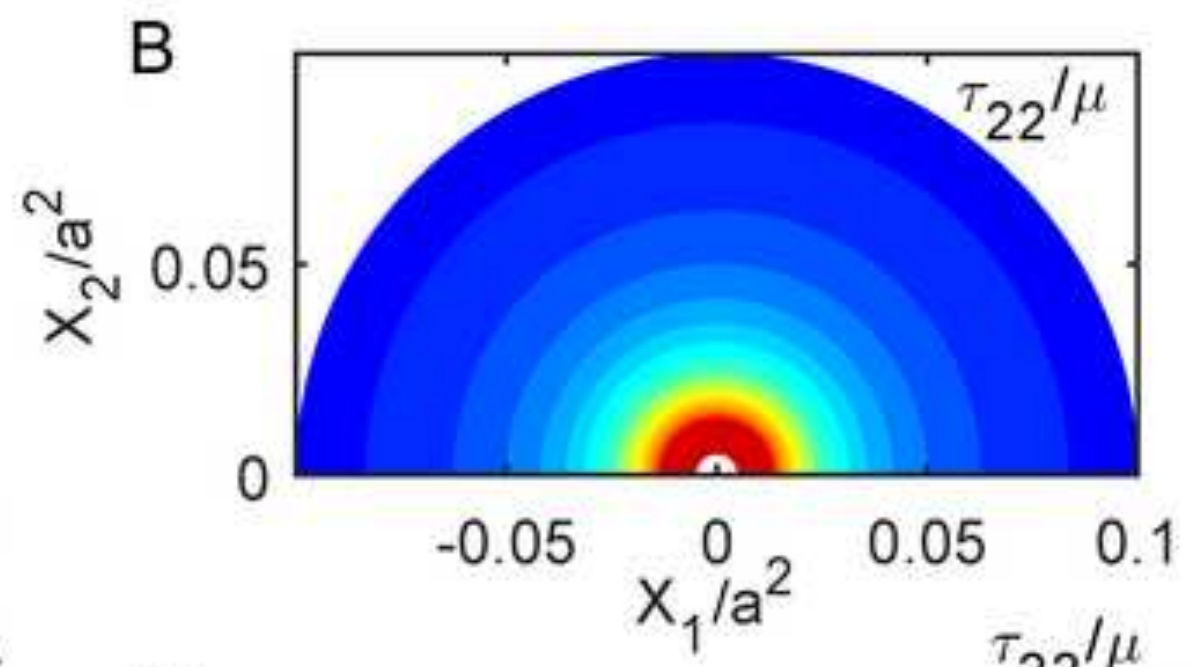

$12 / \mu$

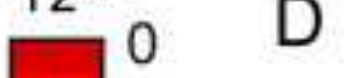

D

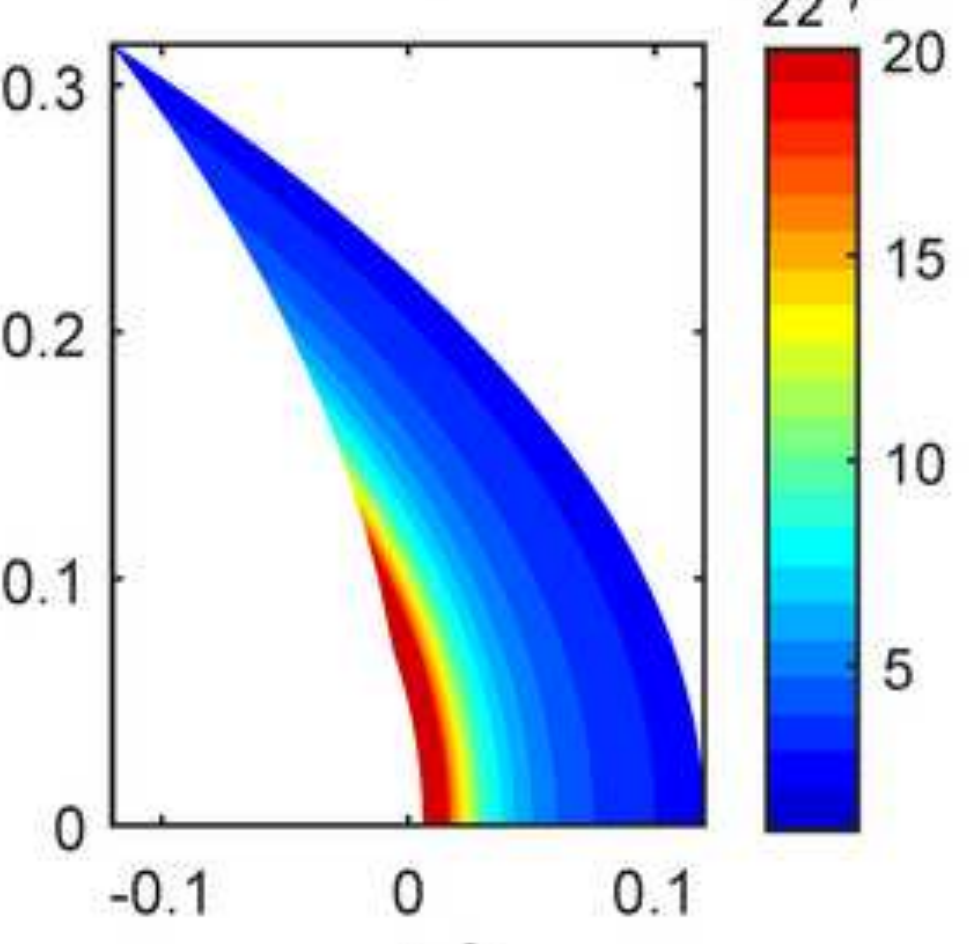

$\mathrm{y}_{1} / \mathrm{a}^{2}$ 

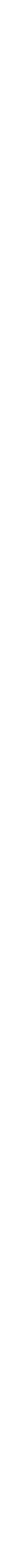

\section{.}

(


$\Delta$

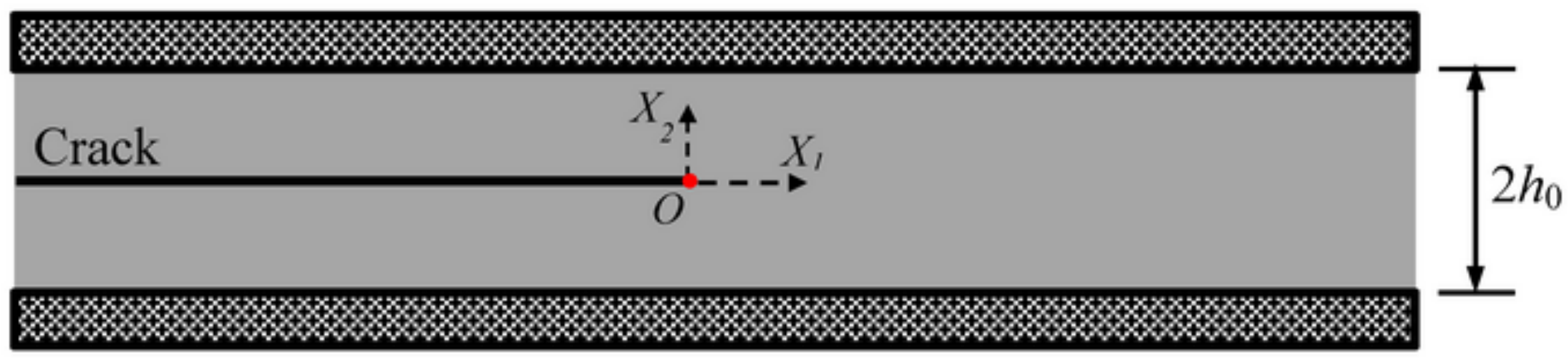

$\Delta$ 